\title{
HARISH-CHANDRA BIMODULES OVER QUANTIZED SYMPLECTIC SINGULARITIES
}

\begin{abstract}
IVAN LOSEV
ABstraCt. In this paper we classify the irreducible Harish-Chandra bimodules with full support over filtered quantizations of conical symplectic singularities under the condition that none of the slices to codimension 2 symplectic leaves has type $E_{8}$. More precisely, consider the quantization $\mathcal{A}_{\lambda}$ with parameter $\lambda$. We show that the top quotient $\overline{\mathrm{HC}}\left(\mathcal{A}_{\lambda}\right)$ of the category of Harish-Chandra $\mathcal{A}_{\lambda}$-bimodules embeds into the category of representations of the algebraic fundamental group, $\Gamma$, of the open leaf. The image coincides with the representations of $\Gamma / \Gamma_{\lambda}$, where $\Gamma_{\lambda}$ is a normal subgroup of $\Gamma$ that can be recovered from the quantization parameter $\lambda$ combinatorially. As an application of our results, we describe the Lusztig quotient group in terms of the geometry of the normalization of the orbit closure in almost all cases.
\end{abstract}

To the memory of Ernest Borisovich Vinberg.

\section{INTRODUCTION}

1.1. Harish-Chandra bimodules over quantizations of symplectic singularities. The goal of this paper is to study Harish-Chandra bimodules over quantizations of conical symplectic singularities.

Let us start by defining Harish-Chandra bimodules in the general setting of filtered quantizations of graded Poisson algebras.

Let $A$ be a finitely generated commutative associative unital algebra. Suppose that $A$ is equipped with two additional structures: an algebra grading $A=\bigoplus_{i=0}^{\infty} A_{i}$ such that $A_{0}=\mathbb{C}$ and a Poisson bracket $\{\cdot, \cdot\}$ of degree $-d$, where $d \in \mathbb{Z}_{>0}$, which, by definition, means that $\left\{A_{i}, A_{j}\right\} \subset A_{i+j-d}$ for all $i, j$. By a filtered quantization of $A$ we mean a pair $(\mathcal{A}, \iota)$ of

- a filtered associative algebra $\mathcal{A}=\bigcup_{i \geqslant 0} \mathcal{A}_{\leqslant i}$ such that $\left[\mathcal{A}_{\leqslant i}, \mathcal{A}_{\leqslant j}\right] \subset \mathcal{A}_{\leqslant i+j-d}$,

- a graded Poisson algebra isomorphism $\iota:$ gr $\mathcal{A} \stackrel{\sim}{\rightarrow} A$.

Following [L1, Section 2.5], by a Harish-Chandra (shortly, HC) $\mathcal{A}$-bimodule we mean an $\mathcal{A}$-bimodule $\mathcal{B}$ that can be equipped with an increasing exhaustive bimodule filtration $\mathcal{B}=\bigcup_{j} \mathcal{B}_{\leqslant j}$ such that $\left[\mathcal{A}_{\leqslant i}, \mathcal{B}_{\leqslant j}\right] \subset \mathcal{B}_{\leqslant i+j-d}$ (which implies that the actions of $A$ on gr $\mathcal{B}$ from the left and from the right coincide) and gr $\mathcal{B}$ is a finitely generated $A$-module. Such a filtration will be called good. For example, the regular bimodule $\mathcal{A}$ is HC.

The most classical example here is when $\mathcal{A}=U(\mathfrak{g})$ for a semi-simple Lie algebra $\mathfrak{g}$, here $d=1$. A Harish-Chandra bimodule is the same thing as a finitely generated $U(\mathfrak{g})$ bimodule with locally finite adjoint action of $\mathfrak{g}$. These bimodules are extensively studied in Lie representation theory.

The algebras $\mathcal{A}$ we are interested in are filtered quantizations of conical symplectic singularities.

MSC 2010: 16G99, 16W70, 17B35. 
Let us recall the definition of a conical symplectic singularity. Let $Y$ be a normal Poisson algebraic variety such that the Poisson bracket on the smooth locus $Y^{\text {reg }}$ is non-degenerate. Let $\omega$ denote the corresponding symplectic form on $Y^{\text {reg }}$. Following Beauville, $[\mathrm{B}$, we say that $Y$ has symplectic singularities if there is a resolution of singularities $\rho: \tilde{Y} \rightarrow Y$ such that $\rho^{*} \omega$ extends to a regular 2 -form on $\tilde{Y}$. We say that a variety $Y$ with symplectic singularities is a conical symplectic singularity if it is equipped with an action of the one-dimensional torus $\mathbb{C}^{\times}$such that

- $\mathbb{C}^{\times}$contracts $Y$ to a single point (and so $Y$ is automatically affine),

- and the degree of the Poisson bracket on $\mathbb{C}[Y]$ is $-d$ for $d \in \mathbb{Z}_{>0}$.

In particular, $A:=\mathbb{C}[Y]$ is a graded Poisson algebra as above.

Examples of conical symplectic singularities include the following.

(1) The nilpotent cone $\mathcal{N}$ in a semisimple Lie algebra $\mathfrak{g}$. More generally, let $\mathbb{O} \subset \mathfrak{g}$ be a nilpotent orbit and $\tilde{\mathbb{O}}$ be its $G$-equivariant cover (where $G$ stands for the simply connected group with Lie algebra $\mathfrak{g})$. Then the algebra $\mathbb{C}[\tilde{\mathbb{O}}]$ is finitely generated and $Y:=\operatorname{Spec}(\mathbb{C}[\tilde{\mathbb{O}}])$ is a conical symplectic singularity. For $\tilde{\mathbb{O}}=\mathbb{O}$ this was observed in [B] based on prior results of Panyushev. The general case follows from there, see Lemma 2.5.

(2) Let $V$ be a symplectic vector space and $\Gamma$ be a finite group of linear symplectomorphisms of $V$. Then $Y:=V / \Gamma$ is a conical symplectic singularity, [B].

There are many other examples of conical symplectic singularities (preimages of Slodowy slices in $\operatorname{Spec}(\mathbb{C}[\tilde{\mathbb{O}}])$, affine Nakajima and hypertoric varieties, etc.) but only (1) and (2) are important for the present paper.

For a general conical symplectic singularity $Y$ the filtered quantizations of $Y$ (i.e., of $\mathbb{C}[Y])$ were classified in [L8. The result can be stated as follows - we will recall it in more detail below in Section 1.3. There is a finite dimensional $\mathbb{C}$-vector space $\mathfrak{h}_{Y}^{*}$ defined over $\mathbb{Q}$ and a finite crystallographic reflection group $W_{Y}$ acting on $\mathfrak{h}_{Y}^{*}$ such that the filtered quantizations of $Y$ are in a natural one-to-one correspondence with $\mathfrak{h}_{Y}^{*} / W_{Y}$. We will write $\mathcal{A}_{\lambda}$ for the filtered quantization corresponding to $\lambda \in \mathfrak{h}_{Y}^{*}$.

In the examples of conical symplectic singularities mentioned above we get algebras of great interest for Representation theory. When $Y$ is the nilpotent cone in $\mathfrak{g}$, its filtered quantizations are the central reductions of $U(\mathfrak{g})$, while for $Y:=\operatorname{Spec}(\mathbb{C}[\tilde{\mathbb{O}}])$ we get interesting Dixmier algebras in the sense of Vogan, $[\mathrm{V}]$. In the case when $Y=V / \Gamma$ we get spherical symplectic reflection algebras of Etingof and Ginzburg, EG.

The goal of this paper is to classify irreducible Harish-Chandra $\mathcal{A}_{\lambda}$-bimodules that are faithful as left or, equivalently, right modules (both conditions are equivalent to the condition that the associated variety of the bimodule coincides with $Y$, we will recall associated varieties in Section 2.4). Below we will say that such $\mathrm{HC}$ bimodules are of full support. One could hope that the classification in this case will shed some light on that of $\mathrm{HC}$ bimodules with arbitrary associated varieties. We note that the opposite case to what we consider is of finite dimensional irreducible bimodules. Here the classification reduces to that of finite dimensional irreducible modules and very much depends on the algebra. In contrast, the classification of irreducible $\mathrm{HC}$ bimodules with full support is geometric, as we will see below. One should expect that the case of general associated varieties interpolates between the two extreme cases.

The classification of irreducible HC bimodules with full support is known in many cases. For example, the result for $Y=\mathcal{N}$ is classical - it will be recalled below in Section 5.1 - 
as this case turns out to be important for the classification in the general case. Various special cases and partial results for $Y=\operatorname{Spec}(\mathbb{C}[\mathbb{O}])$ were obtained in $[$ L1, LO, L6]. The case of $Y=V / \Gamma$ was considered in [L3] and then in [S]. In the latter paper a complete classification was obtained in the case when $V=U \oplus U^{*}$ and $\Gamma$ acts on $U$ as a complex reflection group.

However, even in some simple cases, most notably for the Kleinian singularities $V / \Gamma$, where $\operatorname{dim} V=2$ and $\Gamma$ is not cyclic, the classification is not known. It turns out that this case is crucial for understanding the case of general $Y$. We consider the Kleinian case in the next section.

1.2. Results for quantizations of Kleinian singularities. So let $Y=\mathbb{C}^{2} / \Gamma$. Recall that, up to conjugation in $\mathrm{SL}_{2}(\mathbb{C})$, the subgroups $\Gamma$ are classified by the type ADE Dynkin diagrams. In particular, to $\Gamma$ we can assign the Cartan space $\mathfrak{h}_{\Gamma}$ and the Weyl group $W_{\Gamma}$ (of the corresponding ADE type). We have $\mathfrak{h}_{Y}^{*}=\mathfrak{h}_{\Gamma}^{*}, W_{Y}=W_{\Gamma}$.

Quantizations of $Y$ were extensively studied in the past with various constructions given in [CBH] (a special case of the general symplectic reflection algebra construction), [H] (as a quantum Hamiltonian reduction), [P] (as the central reduction of a suitable finite $\mathrm{W}$-algebra). All these constructions give the same quantizations, see [L4, Theorems $5.3 .1,6.2 .2]$.

Let us describe the classification of irreducible $\mathrm{HC}$ bimodules with full support in this case. We start with a discussion of quantization parameters, see Example 2.8 for details. We have an affine isomorphism between $\mathfrak{h}_{\Gamma}^{*}$ and the affine subspace $(\mathbb{C} \Gamma)_{1}^{\Gamma} \subset(\mathbb{C} \Gamma)^{\Gamma}$ consisting of elements $c$ of the form $1+\sum_{\gamma \neq 1} c_{\gamma} \gamma$. Namely to $c \in(\mathbb{C} \Gamma)_{1}^{\Gamma}$ we assign $\lambda_{c} \in \mathfrak{h}_{Y}^{*}$ with $\left\langle\lambda_{c}, \alpha_{i}^{\vee}\right\rangle=\operatorname{tr}_{N_{i}}(c)$. Here we write $\alpha_{i}^{\vee}$ for a simple coroot in $\mathfrak{h}_{\Gamma}$ and $N_{i}$ for the corresponding nontrivial irreducible representation of $\Gamma$.

Now we proceed to a conjectural classification result for irreducible $\mathrm{HC} \mathcal{A}_{\lambda}$-bimodules with full support. Consider the affine Weyl group $W_{\Gamma}^{a}:=W_{\Gamma} \ltimes \Lambda_{r}$, where $\Lambda_{r}$ is the root lattice in $\mathfrak{h}_{\Gamma}^{*}$. The group $W_{\Gamma}^{a}$ naturally acts on $\mathfrak{h}_{\Gamma}^{*}$ by affine transformations.

Conjecture 1.1. The following claims are true:

(1) For each $c \in(\mathbb{C} \Gamma)_{1}^{\Gamma}$, there is a minimal normal subgroup $\Gamma_{c} \subset \Gamma$ such that $W_{\Gamma}^{a} \lambda_{c}$ contains $\lambda_{c^{\prime}}$ with $c^{\prime} \in \mathbb{C} \Gamma_{c}(\subset \mathbb{C} \Gamma)$.

(2) The irreducible $\mathrm{HC} \mathcal{A}_{\lambda_{c}}$-bimodules with full support are in bijection with the irreducible representations of $\Gamma / \Gamma_{c}$.

Theorem 1.2. Conjecture 1.1 is true when $\Gamma$ is not of type $E_{8}$.

Under the same restriction, we can also describe the top quotient of the category of HC bimodules as a tensor category, Theorem 5.1.

At this point, we do not know what happens in the $E_{8}$-case. Clearly, (1) should not be difficult to check. On the other hand, the conclusion of (2) is true for some normal subgroup of $\Gamma$, we just do not know which of the three normal subgroups to take. What makes type $E_{8}$ special is that the corresponding Kleinian group is not solvable.

We also note that the type A case $(=$ cyclic $\Gamma$ ) of Conjecture 1.1 was proved in $[S]$.

The most essential ingredient of the proof of Theorem 1.2 is to relate the HC bimodules over $\mathcal{A}_{\lambda}$ and over the central reduction $\mathcal{U}_{\lambda}$ of $U(\mathfrak{g})$ (where $\mathfrak{g}$ is a semisimple Lie algebra of the same type as $\Gamma$ ) corresponding to $\lambda$. This is a special case of the general extension result for $\mathrm{HC}$ bimodules that also allows to reduce the classification in general to that in the Kleinian case. 
1.3. Results for quantizations of symplectic singularities. Now assume that $Y$ is a general conical symplectic singularity. Pick $\lambda \in \mathfrak{h}_{Y}^{*}$ and let $\mathcal{A}_{\lambda}$ be the corresponding filtered quantization. Consider the algebraic fundamental group $\Gamma$ of $Y^{\text {reg }}$. Recall that this group is the pro-finite completion of $\pi_{1}\left(Y^{r e g}\right)$. The finite index subgroups of $\Gamma$ are in one-to-one correspondence with finite etale covers of $Y^{r e g}$. By a result of Namikawa, [N4], $\Gamma$ is a finite group. Any finite dimensional representation of $\pi_{1}\left(Y^{r e g}\right)$ factors through $\Gamma$.

More precisely, we will see that $\lambda$ defines a normal subgroup of $\Gamma$, to be denoted by $\Gamma_{\lambda}$, and the set of irreducible HC bimodules with full support is in a bijection with $\operatorname{Irr}\left(\Gamma / \Gamma_{\lambda}\right)$, the set of isomorphism classes of irreducible representations of $\Gamma / \Gamma_{\lambda}$.

Let us explain how to construct the normal subgroup $\Gamma_{\lambda}$. By a result of Kaledin, $[\mathrm{K}]$, $Y$ has finitely many symplectic leaves. Let $\mathcal{L}_{1}, \ldots, \mathcal{L}_{k}$ be the codimension 2 leaves. Let $\Sigma_{1}, \ldots, \Sigma_{k}$ be formal slices through $\mathcal{L}_{1}, \ldots, \mathcal{L}_{k}$. Then $\Sigma_{i}=\mathbb{D}^{2} / \Gamma_{i}$, where we write $\mathbb{D}^{2}$ for $\operatorname{Spec}(\mathbb{C}[[x, y]])$. So we can consider the corresponding Cartan space $\tilde{\mathfrak{h}}_{i}^{*}$ for $\Gamma_{i}$. The fundamental group $\pi_{1}\left(\mathcal{L}_{i}\right)$ acts on $\tilde{\mathfrak{h}}_{i}^{*}$ by monodromy. Let $\mathfrak{h}_{i}^{*}:=\left(\tilde{\mathfrak{h}}_{i}^{*}\right)^{\pi_{1}\left(\mathcal{L}_{i}\right)}$. Then we have $\mathfrak{h}_{Y}^{*}=\bigoplus_{i=0}^{k} \mathfrak{h}_{i}^{*}$, where $\mathfrak{h}_{0}^{*}:=H^{2}\left(Y^{r e g}, \mathbb{C}\right)$, see [L8, Lemma 2.8].

Let us write $\lambda_{i}$ for the component of $\lambda$ in $\mathfrak{h}_{i}^{*} \subset \tilde{\mathfrak{h}}_{i}^{*}$ and let $c_{i} \in \mathbb{C} \Gamma_{i}$ be the element corresponding to $\lambda_{i}$ as explained in the previous section. Let us write $\Gamma_{i, \lambda}$ for the normal subgroup $\Gamma_{i, c_{i}}$ from Conjecture 1.1.

Now note that we have a natural group homomorphism $\Gamma_{i}=\pi_{1}^{a l g}\left(\Sigma_{i} \backslash\{0\}\right) \rightarrow \Gamma$ induced by the inclusion $\Sigma_{i} \backslash\{0\} \hookrightarrow Y^{\text {reg }}$. Let $\Gamma_{\lambda}$ be the minimal normal subgroup of $\Gamma$ containing the images of all $\Gamma_{i, \lambda}$.

Theorem 1.3. Suppose that Conjecture 1.1 holds for all $\Gamma_{i}, i=1, \ldots, k$. Then there is a bijection between the irreducible $H C \mathcal{A}_{\lambda}$-bimodules with full support and the irreducible $\Gamma / \Gamma_{\lambda}$-modules.

There is a stronger version on the level of tensor categories, Theorem 6.1.

So we have a full classification of irreducible $\mathrm{HC} \mathcal{A}_{\lambda}$-bimodules with full support in the case when $Y$ has no two-dimensional slices of type $E_{8}$. This is the case in the majority of interesting examples. For instance, if we are dealing with $Y=\operatorname{Spec}(\mathbb{C}[\tilde{\mathbb{O}}])$, see Example (2) in Section 1.1, then this assumption fails precisely when $Y$ is the nilpotent cone in the Lie algebra of type $E_{8}$. Of course, in that case the classification is also known.

Remark 1.4. More generally, for two different filtered quantizations $\mathcal{A}_{\lambda^{\prime}}, \mathcal{A}_{\lambda}$ of $Y$ one can consider $\mathrm{HC} \mathcal{A}_{\lambda^{\prime}}-\mathcal{A}_{\lambda^{-}}$-bimodules and ask to classify such irreducible bimodules with full support. The situation there is more complicated than in the case of $\lambda^{\prime}=\lambda$, we have partial results on the classification (including a complete classification when $\mathfrak{h}_{0}^{*}=0$ ) that we will explain sketching required modifications in Section 6.2. One reason we chose to omit complete proofs in the general case is that the case $\lambda^{\prime}=\lambda$ is much less technical but also is more important for applications, including those in Lie representation theory.

1.4. Applications and variants. One application of Theorem 1.3 is a geometric interpretation of Lusztig's quotients, [Lu, Section 13], for almost all cases (with the exception of one case in $E_{7}$ and three in $E_{8}$ ). These finite groups were introduced by Lusztig in his work on computing the characters for finite groups of Lie type. Namely, from a two-sided cell $c$ in a Weyl group $W$ Lusztig has produced a finite group $\overline{\mathbf{A}}_{c}$. He also established a connection of this group to nilpotent orbits, as follows.

Let $\mathfrak{g}$ be a semisimple Lie algebra with Weyl group $W$. Then the two-sided cells in $W$ are in one-to-one correspondence with the so called special orbits in $\mathfrak{g}$. Let $\mathbb{O}_{c}$ denote 
the orbit corresponding to $c$. Lusztig has proved that $\overline{\mathbf{A}}_{c}$ can be realized as a quotient of the component group $\mathbf{A}\left(\mathbb{O}_{c}\right)$, that is the $G$-equivariant fundamental group of $\mathbb{O}_{c}$, where $G:=\operatorname{Ad}(\mathfrak{g})$.

The quotients $\overline{\mathbf{A}}_{c}$ were further studied in a number of papers including [LO]. There the author and Ostrik computed $\overline{\mathbf{A}}_{c}$ in terms of the two-sided $W$-module $[c]$ corresponding to $c$ and the Springer representation of $W \times \mathbf{A}\left(\mathbb{O}_{c}\right)$ associated to $\mathbb{O}_{c}$. Using this, we have identified the semi-simple part of the subquotient of $\mathrm{HC}\left(\mathcal{U}_{\rho}\right)$ corresponding to $\mathbb{O}_{c}$ (this subquotient categorifies $[c])$ with the category $\operatorname{Sh}^{\overline{\mathbf{A}}_{c}}\left(Y_{c} \times Y_{c}\right)$, where $Y_{c}$ is the category of finite dimensional modules over the $\mathrm{W}$-algebra corresponding to $\mathbb{O}_{c}$. Below in Section 7 we will use this result from [LO] and Theorem 1.3 to show that $\overline{\mathbf{A}}_{c}=\Gamma / \Gamma_{\lambda}$, where $\Gamma=\pi_{1}\left(\mathbb{O}_{c}\right)$ and $\lambda$ is suitable quantization parameter for $\mathbb{C}\left[\mathbb{O}_{c}\right]$ (that exists for all $\mathbb{O}_{c}$ but the four mentioned above). This gives a new description of $\overline{\mathbf{A}}_{c}$ basically in terms of the geometry of $\operatorname{Spec}\left(\mathbb{C}\left[\mathbb{O}_{c}\right]\right)$. The main results of Section 7 are Propositions 7.3 and 7.4 .

We now mention some subsequent work. In [LMBM] we give a new definition of unipotent Harish-Chandra bimodules over semisimple Lie algebras and apply Theorem 1.3 to classify and study them. And in [LY], we prove an analog of Theorem 1.3 for irreducible Harish-Chandra modules over quantizations of $\mathbb{C}[\mathbb{O}]$, where $\mathbb{O}$ is a nilpotent orbit in a semisimple Lie algebra satisfying $\operatorname{codim}_{\overline{\mathbb{O}}} \partial \mathbb{O} \geqslant 4$.

Acknowledgements. I would like to thank Pavel Etingof, George Lusztig, Dmytro Matvieievskyi and Victor Ostrik for stimulating discussions. I would also like to thank Dmytro Matvieievskyi and Shilin Yu for the many comments that allowed me to improve the exposition. This work has been funded by the Russian Academic Excellence Project '5-100'. This work was also partially supported by the NSF under grant DMS-1501558. This paper is dedicated to the memory of my advisor, Ernest Borisovich Vinberg, who sadly passed away in May 2020.

\section{Preliminaries}

2.1. Non-commutative period map. In this section we will discuss quantizations of smooth symplectic algebraic varieties and their important invariant, the non-commutative period, following [BK, L4].

Let $X$ be a symplectic algebraic variety. So $\mathcal{O}_{X}$ is a Poisson sheaf of algebras. By a formal quantization of $X$ we mean a pair $\left(\mathcal{D}_{h}, \iota\right)$, where

- $\mathcal{D}_{h}$ is a sheaf in Zariski topology of $\mathbb{C}[[h]]$-algebras on $X$ that is $\mathbb{C}[[h]]$-flat, and complete and separated in the $h$-adic topology,

- and $\iota: \mathcal{D}_{h} / h \mathcal{D}_{h} \stackrel{\sim}{\rightarrow} \mathcal{O}_{X}$ is an isomorphism of sheaves of Poisson algebras on $X$.

We note that in the case when $X$ is affine, to give a formal quantization of $X$ is the same as to give a formal quantization of $\mathbb{C}[X]$.

Bezrukavnikov and Kaledin in [BK, Section 4] defined an invariant of $\mathcal{D}_{h}$ called the non-commutative period that lies in $H^{2}(X, \mathbb{C}[[h]])$. Let us explain the construction as we will need it below.

The first step in the construction is passing from a quantization $\mathcal{D}_{h}$ to its quantum jet bundle, to be denoted by $\mathrm{J}^{\infty} \mathcal{D}_{h}$, that is a pro-coherent sheaf of $\mathbb{C}[[h]]$-algebras on $X$ equipped with a flat connection.

Let us start with recalling the usual jet bundle $\mathrm{J}^{\infty} \mathcal{O}_{X}$. Consider $X \times X$ with the projections $p_{1}, p_{2}: X \times X \rightarrow X$. By the jet bundle $\mathrm{J}^{\infty} \mathcal{O}_{X}$ we mean $p_{1 *}\left(\widehat{\mathcal{O}}_{\Delta}\right)$, where we write $\widehat{\mathcal{O}}_{\Delta}$ for the completion of $\mathcal{O}_{X \times X}$ along the diagonal $\Delta$. This is a pro-coherent 
sheaf on $\mathcal{O}_{X}$ whose fiber at $x \in X$ is the completion $\mathcal{O}_{X}^{\wedge}$ at $x$. This bundle comes with a flat connection $\nabla$ (derivatives along the first copy of $X$ ). The subsheaf of flat sections $\left(\mathrm{J}^{\infty} \mathcal{O}_{X}\right)^{\nabla}$ is identified with $\mathcal{O}_{X}$ via $p_{2}^{*}$. Finally, note that $\mathrm{J}^{\infty} \mathcal{O}_{X}$ comes with a natural $\mathcal{O}_{X}$-linear Poisson structure.

Now let $\mathcal{D}_{h}$ be a formal quantization of $\mathcal{O}_{X}$. Then we can form the quantum jet bundle $\mathrm{J}^{\infty} \mathcal{D}_{h}$ : we consider the completion of $\mathcal{O}_{X} \otimes \mathcal{D}_{h}$ along the diagonal $\Delta$, denote this sheaf by $\widehat{\mathcal{D}}_{h, \Delta}$. Then $\mathrm{J}^{\infty} \mathcal{D}_{h}:=p_{1 *} \widehat{\mathcal{D}}_{\hbar, \Delta}$. Again, this is a pro-coherent sheaf on $X$ with a flat connection. The sheaf of flat sections of this connection is $\mathcal{D}_{h}$ and $\mathrm{J}^{\infty} \mathcal{D}_{h} /(h)=\mathrm{J}^{\infty} \mathcal{O}_{X}$.

Let $\mathbb{A}_{h}$ denote the formal Weyl algebra in $\operatorname{dim} X$-variables, the unique formal quantization of the Poisson algebra $\mathbb{C}\left[\left[x_{1}, \ldots, x_{n}, y_{1}, \ldots, y_{n}\right]\right]$ (with the standard Poisson bracket), where $\operatorname{dim} X=2 n$. The sheaf $\mathrm{J}^{\infty} \mathcal{D}_{h}$ defines a torsor over the Harish-Chandra pair (Aut $\mathbb{A}_{h}$, Der $\mathbb{A}_{h}$ ). The sheaf $J^{\infty} \mathcal{D}_{h}$ is the associated bundle of this torsor with fiber $\mathbb{A}_{h}$. The assignment sending $\mathcal{D}_{h}$ to that torsor is a bijection between

- the set of isomorphism classes of quantizations,

- and the set of isomorphism classes of Harish-Chandra torsors over $\left(\right.$ Aut $\mathbb{A}_{h}$, Der $\left.\mathbb{A}_{h}\right)$ that specialize to the torsor of formal coordinate systems at $h=0$.

The map $h^{-1} a \mapsto h^{-1}[a, \cdot]$ is an epimorphism $h^{-1} \mathbb{A}_{\hbar} \rightarrow$ Der $\mathbb{A}_{\hbar}$ with kernel $h^{-1} \mathbb{C}[[h]]$. The exact sequence of Lie algebras

$$
0 \rightarrow h^{-1} \mathbb{C}[[h]] \rightarrow h^{-1} \mathbb{A}_{h} \rightarrow \operatorname{Der} \mathbb{A}_{h} \rightarrow 0
$$

lifts to an exact sequence of Harish-Chandra pairs

$$
0 \rightarrow\left(h^{-1} \mathbb{C}[[h]], h^{-1} \mathbb{C}[[h]]\right) \rightarrow \mathrm{G} \rightarrow\left(\text { Aut } \mathbb{A}_{h}, \text { Der } \mathbb{A}_{h}\right) \rightarrow 0 .
$$

Here the first torsor corresponds to the additive group $h^{-1} \mathbb{C}[[h]]$ and $\mathrm{G}$ is defined in $[\mathrm{BK}$, Section 3.2]. The exact sequence gives rise to the map Per: Quant $(X) \rightarrow H_{D R}^{2}(X, \mathbb{C}[[h]])$, where we write Quant $(X)$ for the set of isomorphism classes of formal quantizations of $X$. This map sends a quantization $\mathcal{D}_{h}$ to the obstruction class for lifting the corresponding Harish-Chandra torsor to a G-torsor. The degree 0 term of $\operatorname{Per}\left(\mathcal{D}_{h}\right)$ is the class of the symplectic form $\omega$ on $X$. By the construction, $\operatorname{Per}\left(\mathcal{D}_{h}\right) \bmod h^{2}$ is recovered from $\mathcal{D}_{h} /\left(h^{2}\right)$ together with a "non-commutative Poisson bracket" $\{\cdot, \cdot\}$ induced by the Lie bracket on $\mathcal{D}_{h} /\left(h^{3}\right)$.

We will be interested in the situation when $\mathbb{C}^{\times}$acts on $X$ with $t . \omega=t^{d} \omega$ for $d \in \mathbb{Z}_{>0}$. Of course, here the cohomology class of $\omega$ is 0 . We say that a formal quantization $\mathcal{D}_{h}$ is graded if the action of $\mathbb{C}^{\times}$on $\mathcal{O}_{X}$ lifts to an action of $\mathbb{C}^{\times}$on $\mathcal{D}_{h}$ by $\mathbb{C}$-algebra automorphisms such that $t . h=t^{d} h$ for $t \in \mathbb{C}^{\times}$and $\iota: \mathcal{D}_{h} / h \mathcal{D}_{h} \stackrel{\sim}{\rightarrow} \mathcal{O}_{X}$ is $\mathbb{C}^{\times}$-equivariant. It was shown in [L4, Section 2.3] that if $\mathcal{D}_{h}$ is graded, then $\operatorname{Per}\left(\mathcal{D}_{h}\right) \in h H_{D R}^{2}(X)$.

The construction of the period generalizes to the relative situation, BK, Section 4]. Let $S$ be a scheme over $\mathbb{C}$. We will mostly be interested in the case when $S=\operatorname{Spec}\left(\mathbb{C}[t] /\left(t^{2}\right)\right)$. Let $X$ be a smooth symplectic scheme (of finite type) over $S$ (meaning, in particular, that now $\omega \in \Omega^{2}(X / S)$ ), let $\pi: X \rightarrow S$ be the corresponding morphism. The notion of a formal quantization still makes sense but now $\mathcal{D}_{h}$ is a sheaf of $\pi^{-1} \mathcal{O}_{S}[[h]]$-algebras and $\iota$ is $\pi^{-1} \mathcal{O}_{S^{-}}$-linear. Here we write $\pi^{-1}$ for the sheaf-theoretic pullback. The set of isomorphism classes of the formal quantizations of $X$ will be denoted by Quant $(X / S)$. To $\mathcal{D}_{h}$ we can assign its period $\operatorname{Per}\left(\mathcal{D}_{h}\right) \in H_{D R}^{2}(X / S)[[h]]$ in the same way as before.

We will need to understand the behavior of the period under regluing. Namely, let us take a graded formal quantization $\mathcal{D}_{h}$. Cover $X$ with $\mathbb{C}^{\times}$-stable open affine subsets $U_{i}$ and let us write $U_{i j}$ for $U_{i} \cap U_{j}$. Let us pick a 1-cocycle $\theta=\left(\theta_{i j}\right)$ of $\mathbb{C}^{\times}$-equivariant 
$\mathbb{C}[[h]]$-linear automorphisms of $\left.\mathcal{D}_{h}\right|_{U_{i j}}$. In particular, $\theta_{j i}=\theta_{i j}^{-1}$ and we have the equality $\theta_{i k}=\theta_{i j} \theta_{j k}$ of automorphisms of $\left.\mathcal{D}_{h}\right|_{U_{i j k}}$. We can form a new quantization $\mathcal{D}_{h}^{\theta}$ obtained from $\mathcal{D}_{h}$ by twisting with $\theta$. We want to relate the periods $\operatorname{Per}\left(\mathcal{D}_{h}\right)$ and $\operatorname{Per}\left(\mathcal{D}_{h}^{\theta}\right)$.

Note that $\theta_{i j}=\exp \left(h \delta_{i j}\right)$, where $\delta_{i j}$ is a derivation $\left.\mathcal{D}_{h}\right|_{U_{i j}}$ of degree $-d$. Let $\delta_{i j}^{0}$ denote $\delta_{i j}$ modulo $h$. This is a symplectic vector field on $U_{i j}$ of degree $-d$. Let $\alpha_{i j}$ be the corresponding 1-form (obtained by pairing $\delta_{i j}$ and $\omega$ ). Note that $\alpha_{i j}$ is closed and has degree 0 . The forms $\alpha_{i j}$ form a Čech and hence a Čech-De Rham cocycle. Let $[\alpha]$ denote its class in $H_{D R}^{2}(X)$.

Lemma 2.1. We have $\operatorname{Per}\left(\mathcal{D}_{h}^{\theta}\right)=\operatorname{Per}\left(\mathcal{D}_{h}\right)+h[\alpha]$.

Proof. Since both quantizations are graded, we have $\operatorname{Per}\left(\mathcal{D}_{h}^{\theta}\right), \operatorname{Per}\left(\mathcal{D}_{h}\right) \in h H_{D R}^{2}(X)$. It remains to show that we have $\operatorname{Per}\left(\mathcal{D}_{h}^{\theta}\right)=\operatorname{Per}\left(\mathcal{D}_{h}\right)+h[\alpha]$ modulo $h^{2}$. For this, consider the scheme $X \times S$ over $S$, where $S:=\operatorname{Spec}\left(\mathbb{C}[t] /\left(t^{2}\right)\right)$, and its quantization $\mathcal{D}_{h} \otimes \mathbb{C}[t] /\left(t^{2}\right)$. We can twist the sheaf $\mathcal{D}_{h} \otimes \mathbb{C}[t] /\left(t^{2}\right)$ with the cocycle $1+t \delta_{i j}$, denote the result by $\left(\mathcal{D}_{h} \otimes \mathbb{C}[t] /\left(t^{2}\right)\right)^{\theta}$. This is a quantization of the corresponding twist $(X \times S)^{\theta}$. The class of the fiberwise symplectic form is $t[\alpha]$. Now consider the specialization of $\left(\mathcal{D}_{h} \otimes \mathbb{C}[t] /\left(t^{2}\right)\right)^{\theta}$ to th. We get the sheaf of algebras over $\mathbb{C}[h] /\left(h^{2}\right)$ that comes with the bracket $\{\cdot, \cdot\}$ induced from the Lie bracket on $\left(\mathcal{D}_{h} \otimes \mathbb{C}[t] /\left(t^{2}\right)\right)^{\theta}$. We have an isomorphism of this specialization with $D_{h}^{\theta} /\left(h^{2}\right)$ that is compatible with the brackets. We conclude that the period of $D_{h}^{\theta}$ $\bmod h^{2}$ coincides with the specialization of that of $\left(\mathcal{D}_{h} \otimes \mathbb{C}[t] /\left(t^{2}\right)\right)^{\theta}$ to $t h$ (where we then need to change the variable $t h$ back to $h)$. The latter is $\operatorname{Per}\left(\mathcal{D}_{h}\right)+t h[\alpha]$. This is equivalent to the formula in the statement of the lemma.

2.2. Classification of quantizations of symplectic varieties. Let us now discuss classification questions and some consequences.

The next claim follows from [BK, Theorem 1.8].

Proposition 2.2. Let $S$ be a $\mathbb{C}$-scheme of finite type and $X$ be a smooth symplectic $S$-scheme of finite type. Assume that $H^{i}\left(X, \mathcal{O}_{X}\right)=0$ for $i=1,2$. Then the map Quant $(X / S) \rightarrow[\omega]+h H_{D R}^{2}(X / S)[[h]]$ is a bijection.

This proposition has the following corollary proved in [L4, Section 2.3].

Corollary 2.3. Let $S=\mathrm{pt}, X$ be as in Proposition 2.2, and we have a $\mathbb{C}^{\times}$-action on $X$ as before. Then the period map gives a bijection between the isomorphism classes of graded formal quantizations and $h H^{2}(X, \mathbb{C})$.

We are going to use Proposition 2.2 to study the derivations of quantizations of affine varieties.

Lemma 2.4. Let $X$ be an affine smooth symplectic variety and $\delta_{0}$ be a Poisson derivation of $\mathbb{C}[X]$. Let $\mathcal{D}_{h}$ be a formal quantization of $X$. Then $\delta_{0}$ lifts to a derivation of the $\mathbb{C}[[h]]$ algebra $\mathcal{D}_{h}$.

Proof. Set $S:=\operatorname{Spec}\left(\mathbb{C}[t] /\left(t^{2}\right)\right)$ and $\tilde{X}:=X \times S$. Thanks to the Gauss-Manin connection, we have an identification $H_{D R}^{2}(\tilde{X} / S)=H_{D R}^{2}(X) \times\left(\mathbb{C}[t] /\left(t^{2}\right)\right)$. We can consider two quantizations of $\tilde{X}$. First, we have $\tilde{\mathcal{D}}_{h}^{1}:=\mathcal{D}_{h} \otimes \mathbb{C}[t] /\left(t^{2}\right)$. Next, we have an automorphism $1+t \delta_{0}$ of $\mathbb{C}[\tilde{X}]$. Let $\tilde{\mathcal{D}}_{h}^{2}$ be the twist of $\tilde{\mathcal{D}}_{h}^{1}$ under this automorphism. Since $1+t \delta_{0}$ acts trivially on the De Rham cohomology, we see that the periods of $\tilde{\mathcal{D}}_{h}^{1}$ and $\tilde{\mathcal{D}}_{h}^{2}$ are the same. Therefore, by Proposition $\left[2.2\right.$, we have a $\mathbb{C}[t] /\left(t^{2}\right) \otimes \mathbb{C}[[h]]$-linear isomorphism $\tilde{\mathcal{D}}_{h}^{1} \stackrel{\sim}{\rightarrow} \tilde{\mathcal{D}}_{h}^{2}$ 
that is the identity modulo $h$. So we have an automorphism of $\tilde{\mathcal{D}}_{h}^{1}$ that modulo $h$ coincides with $1+t \delta_{0}$. We can write $\alpha$ as $\alpha_{0}+t \alpha_{1}$, where $\alpha_{0}, \alpha_{1}$ are maps $\mathcal{D}_{h} \rightarrow \mathcal{D}_{h}$. Then $\alpha_{0}^{-1} \circ \alpha_{1}$ is a derivation of $\mathcal{D}_{h}$ lifting $\delta_{0}$.

2.3. Symplectic singularities, their $\mathbb{Q}$-terminalizations and quantizations. The definition of a conical symplectic singularity as well as basic examples were recalled in Section 1.1. In this section we will study some further properties of conical symplectic singularities and their quantizations.

Let $Y$ be a conical symplectic singularity. Let us recall the notation: $\Gamma, \mathcal{L}_{i}, \Gamma_{i}, \tilde{\mathfrak{h}}_{i}^{*}, i=$ $1, \ldots, k, \mathfrak{h}_{j}^{*}, j=0, \ldots, k$ from Section 1.3 .

First, let us discuss covers. Let $\hat{Y}^{0}$ be a finite étale cover of $Y^{r e g}$. Then $\mathbb{C}\left[\hat{Y}^{0}\right]$ is a finitely generated algebra. This follows from the Stein factorization for $\hat{Y}^{0} \rightarrow Y$. We set $\hat{Y}:=\operatorname{Spec}\left(\mathbb{C}\left[\hat{Y}^{0}\right]\right)$. This is an affine Poisson variety.

The proof of the following lemma was explained to me by Dmytro Matvieievskyi.

Lemma 2.5. The Poisson variety $\hat{Y}$ is a conical symplectic singularity.

Proof. By the construction, $\operatorname{codim}_{\hat{Y}}\left(\hat{Y} \backslash \hat{Y}^{0}\right) \geqslant 2$. So $\hat{Y}^{\text {reg }}$ is symplectic.

Let us show that $\hat{Y}$ has symplectic singularities. By a result of Namikawa, [N1, Theorem $6]$, it is enough to show that $\hat{Y}$ has rational Gorenstein singularities. The latter follows from [Br, Theorem 6.2].

Now to prove that $\hat{Y}$ is conical we just need to observe that the action of $\mathbb{C}^{\times}$on $Y^{\text {reg }}$ lifts to $\hat{Y}^{0}$ perhaps after replacing $\mathbb{C}^{\times}$with a cover.

Let us discuss certain partial Poisson resolutions of $Y: \mathbb{Q}$-factorial terminalizations $(\mathbb{Q}$ terminalizations for short). These are Poisson partial resolutions $\rho: X \rightarrow Y$, where $X$ is normal and has the following two properties:

(1) The variety $X$ is $\mathbb{Q}$-factorial: every Weil divisor of $X$ is $\mathbb{Q}$-Cartier, meaning that some its positive integral multiple is Cartier.

(2) $\operatorname{codim}_{X} X^{\operatorname{sing}} \geqslant 4$. Namikawa proved that, in the present situation, this is equivalent to $X$ being terminal.

The action of $\mathbb{C}^{\times}$on $Y$ then lifts to $X$ by a result of Namikawa. See [L9, Proposition 2.1] for details.

Note that $\rho$ is an isomorphism over $Y^{r e g}$ and is a resolution of singularities over $Y^{\text {sreg }}=$ $Y^{r e g} \cup \bigsqcup_{i=1}^{k} \mathcal{L}_{i}$. Let us record the following fact for the future use, see the proof of [N3, Proposition 1.11].

Lemma 2.6. We have $\mathbb{C}\left[X^{r e g}\right]=\mathbb{C}[Y]$ and $H^{i}\left(X^{\text {reg }}, \mathcal{O}\right)=0$ for $i=1,2$.

Now let us discuss filtered quantizations following [BPW], L8], these results are reviewed, for example, in [L8, Section 3.2]. The space $\mathfrak{h}_{Y}^{*}$ mentioned in Section 1.3] is identified with $H^{2}\left(X^{r e g}, \mathbb{C}\right)$, [N3]. So, by the results recalled in Section 2.2 , to $\lambda \in \mathfrak{h}_{Y}^{*}$ we can assign the graded formal quantization $\mathcal{D}_{\lambda h}^{\circ}$ of $X^{r e g}$. Set $\mathcal{A}_{\lambda h}:=\Gamma\left(\mathcal{D}_{\lambda h}^{\circ}\right)$. Lemma 2.6 then implies that $\mathcal{A}_{\lambda h}$ is a graded formal quantization of $Y$. Let $\mathcal{A}_{\lambda h \text {,fin }}$ denote the subalgebra of $\mathbb{C}^{\times}$-finite elements in $\mathcal{A}_{\lambda h}$. We set $\mathcal{A}_{\lambda}:=\mathcal{A}_{\lambda h, \text { fin }} /(h-1)$.

Let $\iota: X^{r e g} \hookrightarrow X$ denote the natural inclusion. Let us write $\mathcal{D}_{\lambda h}$ for $\iota_{*} \mathcal{D}_{\lambda h}^{\circ}$. This is a graded formal quantization of $X$. Moreover, $X$ has a universal graded Poisson deformation $X_{\mathfrak{h}}$ over $\mathfrak{h}_{Y}^{*}$ and $\mathcal{D}_{\lambda h}=\mathcal{D}_{\mathfrak{h}, h} \otimes_{\mathbb{C}\left[\mathfrak{h}_{Y}^{*}\right][[h]]} \mathbb{C}[[h]]$ where $\mathcal{D}_{\mathfrak{h}, h}$ is the canonical quantization of 
$X_{\mathfrak{h}} / \mathfrak{h}_{Y}^{*}$ and the homomorphism $\mathbb{C}\left[\mathfrak{h}_{Y}^{*}\right][[h]] \rightarrow \mathbb{C}[[h]]$ is given by $h \mapsto h, \alpha \mapsto\langle\alpha, \lambda\rangle h$ for $\alpha \in \mathfrak{h}_{Y}$.

Some quantizations $\mathcal{A}_{\lambda}, \mathcal{A}_{\lambda^{\prime}}$ for different $\lambda, \lambda^{\prime}$ are isomorphic (while $\mathcal{D}_{\lambda h}, \mathcal{D}_{\lambda^{\prime} h}$ are not). To explain when this happens we need the Namikawa-Weyl group $W_{Y}$ defined in [N2]. Recall the simply laced Weyl group $\tilde{W}_{i}$ associated with $\Gamma_{i}$. The group $\pi_{1}\left(\mathcal{L}_{i}\right)$ acts on $\tilde{W}_{i}$ by diagram automorphisms. We set $W_{i}:=\tilde{W}_{i}^{\pi_{1}\left(\mathcal{L}_{i}\right)}$, this is a crystallographic reflection group acting faithfully on $\mathfrak{h}_{i}^{*}$. Then $W_{Y}:=\prod_{i=1}^{k} W_{i}$. It is not difficult to show that $\mathcal{A}_{\lambda} \cong \mathcal{A}_{\lambda^{\prime}}$ if $\lambda^{\prime} \in W_{Y} \lambda$, this follows from [L8, Theorem 3.4].

Example 2.7. Let $\mathfrak{g}$ be a semisimple Lie algebra and $Y=\mathcal{N}$ be the nilpotent cone in $\mathfrak{g}$. Its quantizations are the central reductions of $U(\mathfrak{g})$. Namely, recall that under the HarishChandra isomorphism the center $Z$ of $U(\mathfrak{g})$ gets identified with $\mathbb{C}\left[\mathfrak{h}^{*}\right]^{W}$, where $\mathfrak{h}, W$ are the Cartan space and the Weyl group of $\mathfrak{g}$. For $\lambda \in \mathfrak{h}^{*}$ define the central reduction $\mathcal{U}_{\lambda}$ of $U(\mathfrak{g})$ by $\mathcal{U}_{\lambda}=U(\mathfrak{g}) / U(\mathfrak{g}) \mathfrak{m}_{\lambda}$, where we write $\mathfrak{m}_{\lambda}$ for the maximal ideal of $Z$ corresponding to $\lambda$. We note that $\mathfrak{h}_{Y}=\mathfrak{h}, W_{Y}=W$. Indeed, this reduces to the case when $\mathfrak{g}$ is simple. In that case, we have a unique codimension 2 symplectic leaf a.k.a. the subregular orbit. The slice to that orbit in $Y$ has the same type as $\mathfrak{g}$ when $\mathfrak{g}$ is simply laced and the same type as the unfolding of the diagram of $\mathfrak{g}$ else (for example for type $B_{n}$ for $n>1$ we get $\left.A_{2 n-1}\right)$. In the non-simply laced case, $\pi_{1}$ acts via the group of diagram automorphisms that folds that diagram.

Note that $\mathcal{U}_{\lambda}$ is the filtered quantization of $\mathbb{C}[Y]$ corresponding to $\lambda \in \mathfrak{h}_{Y}^{*}$.

Example 2.8. We proceed with $Y=\mathbb{C}^{2} / \Gamma$, where $\Gamma$ is a finite subgroup of $\mathrm{SL}_{2}(\mathbb{C})$. Pick $c \in(\mathbb{C} \Gamma)_{1}^{\Gamma}$ (recall that this means that $c=1+\sum_{\gamma \neq 1} c_{\gamma} \gamma$ ), where $\gamma \mapsto c_{\gamma}: \mathbb{C} \Gamma \backslash$ $\{1\} \rightarrow \mathbb{C}$ is a $\Gamma$-invariant function. Consider the Crawley-Boevey-Holland algebra $H_{c}:=$ $\mathbb{C}\langle x, y\rangle \# \Gamma /(x y-y x-c)$. Let $e \in \mathbb{C} \Gamma$ be the averaging idempotent. Then we can consider the spherical subalgebra $e H_{c} e$ (with unit $e$ ). It was explained in Section 1.2 how to get $\lambda_{c} \in \mathfrak{h}_{Y}^{*}=\tilde{\mathfrak{h}}_{i}^{*}$ from $c$.

It turns out that we have $\mathcal{A}_{\lambda_{c}} \cong e H_{c} e$ for all $c$. In order to prove this we first note that $\mathcal{A}_{\lambda}$ is obtained from $\mathcal{U}_{\lambda}$ (for simply laced $\mathfrak{g}$ of the same type as $\Gamma$ ) via the quantum slice construction (see e.g. [L7, Section 3.2]) applied to the subregular orbit. The isomorphism $\mathcal{A}_{\lambda_{c}} \cong e H_{c} e$ follows, for example, from [L4, Theorem 6.2.2] combined with [L4, Theorem 5.3.1]. The parameter $\lambda$ is recovered from the quantization uniquely up to the $W_{Y^{-}}$ conjugacy, where $W_{Y}:=\tilde{W}_{i}$.

The construction of Example 2.8 has the following useful and elementary corollary.

Corollary 2.9. Let $\Gamma^{\prime} \subset \Gamma$ be a normal subgroup and assume $c \in(\mathbb{C} \Gamma)_{1}^{\Gamma} \cap \mathbb{C}^{\prime}$. So we have quantizations $\mathcal{A}_{c}^{\prime}$ of $\mathbb{C}^{2} / \Gamma^{\prime}$ and $\mathcal{A}_{c}$ of $\mathbb{C}^{2} / \Gamma$. Then $\Gamma / \Gamma^{\prime}$ acts on $\mathcal{A}_{c}$ by automorphisms and the quantizations $\mathcal{A}_{c}^{\prime}$ and $\left(\mathcal{A}_{c}\right)^{\Gamma / \Gamma^{\prime}}$ of $\mathbb{C}^{2} / \Gamma$ are isomorphic.

Let us explain how to recover $\lambda$ from $\mathcal{A}_{\lambda}$ in the case of a general conical symplectic singularity, [L8, Remark 3.6]. We can write $\lambda$ as $\left(\lambda_{0}, \ldots, \lambda_{k}\right)$ with $\lambda_{i} \in \mathfrak{h}_{i}^{*}$. We are going to explain the meaning of parameters $\lambda_{0}, \ldots, \lambda_{k}$. The parameter $\lambda_{0} \in H^{2}\left(Y^{r e g}, \mathbb{C}\right)$ is the period of the microlocalization $\left.\mathcal{A}_{\lambda h}\right|_{Y^{\text {reg }}}$.

The parameters $\lambda_{i}$ (defined up to $W_{i}$-conjugacy) are recovered from the restriction of $\mathcal{A}_{\lambda h}$ to the formal neighborhood of $y_{i} \in \mathcal{L}_{i}$. Namely, consider the completion $\mathcal{A}_{\lambda h}^{\wedge_{y_{i}}}$ with respect to the maximal ideal that is obtained as the inverse image of the maximal ideal of $y_{i}$ under the projection $\mathcal{A}_{\lambda h} \rightarrow \mathbb{C}[Y]$. 
Now assume that $d$ is even (we can always replace $d$ with a multiple by rescaling the $\mathbb{C}^{\times}$-action). Consider the symplectic vector space $V:=T_{y_{i}} \mathcal{L}_{i}$ and form the homogeneous Weyl algebra $\mathbb{A}_{h}:=T(V)[h] /(u \otimes v-v \otimes u-h \omega(u, v))$ with $V$ in degree $d / 2$. We can also form $\underline{\mathcal{A}}_{\lambda_{i} h}$, the homogeneous version of the quantization of $\mathbb{C}^{2} / \Gamma_{i}$ with parameter $\lambda_{i}$.

The following result is a special case of [L7, Lemma 3.3], it explains the meaning of $\lambda_{i}$ (up to the $W_{i}$-conjugacy).

Lemma 2.10. We have a $\mathbb{C}[[h]]$-linear isomorphism $\mathcal{A}_{\lambda h}^{\wedge_{y_{i}}} \cong\left(\mathbb{A}_{h} \otimes_{\mathbb{C}[h]} \underline{\mathcal{A}}_{\lambda_{i} h}\right)^{\wedge_{0}}$.

Finally, let us explain the classification results for filtered quantizations of $Y$ [L8, Theorem 3.4].

Proposition 2.11. Every filtered quantization of $\mathbb{C}[Y]$ is of the form $\mathcal{A}_{\lambda}$ for some $\lambda \in \mathfrak{h}_{Y}^{*}$.

2.4. Harish-Chandra and Poisson bimodules. Let $X$ be a Poisson scheme. By a coherent Poisson $\mathcal{O}_{X}$-module we mean a coherent sheaf $\mathcal{M}$ of $\mathcal{O}_{X}$-modules equipped with a map of sheaves (of vector spaces) $\{\cdot, \cdot\}: \mathcal{O}_{X} \otimes_{\mathbb{C}} \mathcal{M} \rightarrow \mathcal{M}$ satisfying the Leibnitz and Jacobi identities (that are special cases of (2) and (3) below).

Let $X$ come equipped with an action of $\mathbb{C}^{\times}$that is compatible with the Poisson bracket on $\mathcal{O}_{X}$ in the following way: there is a positive integer $d$ such that $t .\{\cdot, \cdot\}=t^{-d}\{\cdot, \cdot\}$ for all $t \in \mathbb{C}^{\times}$. We say that a coherent Poisson module $\mathcal{M}$ is graded if it is $\mathbb{C}^{\times}$-equivariant (as a coherent sheaf) and $\mathbb{C}^{\times}$rescales the bracket $\mathcal{O}_{X} \otimes \mathcal{M} \rightarrow \mathcal{O}_{X}$ by $t \mapsto t^{-d}$.

Now let $Y$ be a conical symplectic singularity and $X=Y^{r e g}$. In this case we can fully classify graded coherent Poisson modules on $Y^{\text {reg }}$ following [L5]. Recall the finite group $\Gamma=\pi_{1}^{a l g}\left(Y^{r e g}\right)$. Let $\tilde{Y}^{0}$ denote the universal algebraic cover of $Y^{\text {reg }}$ (with Galois group $\Gamma$ ) and $\pi: \tilde{Y}^{0} \rightarrow Y^{r e g}$ be the quotient map. Then we have the following result established in the proof of [L5, Lemma 3.9].

Lemma 2.12. The following statements are true:

(1) Every graded coherent Poisson $\mathcal{O}_{\tilde{Y}^{0}}$-module is the direct sum of several copies of $\mathcal{O}_{\tilde{Y}^{0}}$ (with grading shifts).

(2) The functor $\pi^{*}$ defines an equivalence between the category graded coherent Poisson $\mathcal{O}_{Y^{r e g}}$-modules and the category of $\Gamma$-equivariant graded coherent $\mathcal{O}_{\tilde{Y}^{0}}$-modules. The quasi-inverse is given by $\pi_{*}(\bullet)^{\Gamma}$. In particular, every coherent graded Poisson $\mathcal{O}_{Y^{r e g}}$-module is semisimple. Up to a grading shift, the simple graded coherent Pois-

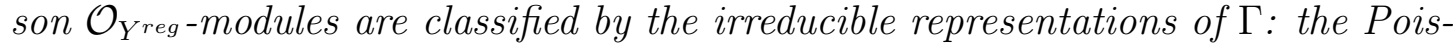
son module corresponding to an irreducible representation $\tau$ is $\operatorname{Hom}_{\Gamma}\left(\tau, \pi_{*} \mathcal{O}_{\tilde{Y}^{0}}\right)$.

Now let $X$ again be a Poisson scheme and $\mathcal{D}_{h}$ be its formal quantization. We will review the definition of a coherent module over a formal quantization below in Section 2.5. Following [L3, Section 3.3] define the notion of a coherent Poisson $\mathcal{D}_{h}$-bimodule. By definition, this is a sheaf $\mathcal{M}_{h}$ of $\mathcal{D}_{h^{-}}$-bimodules on $X$, coherent as a sheaf of left $\mathcal{D}_{h^{-}}$ modules, that is equipped with a bracket map $\{\cdot, \cdot\}: \mathcal{D}_{h} \otimes_{\mathbb{C}[[h]]} \mathcal{M}_{h} \rightarrow \mathcal{M}_{h}$. This bracket map is supposed to satisfy the following conditions (where for local sections $a, b$ of $\mathcal{D}_{\hbar}$ we write $\left.\{a, b\}:=\frac{1}{h}[a, b]\right)$ :

(1) For local sections $a$ of $\mathcal{D}_{\hbar}$ and $m$ of $\mathcal{M}_{\hbar}$ we have $a m-m a=h\{a, m\}$.

(2) The Jacobi identity: $\{\{a, b\}, m\}=\{a,\{b, m\}\}-\{b,\{a, m\}\}$. 
(3) The Leibniz identities:

$$
\begin{aligned}
& \{a b, m\}=\{a, m\} b+a\{b, m\}, \\
& \{a, b m\}=\{a, b\} m+b\{a, m\}, \\
& \{a, m b\}=\{a, m\} b+m\{a, b\} .
\end{aligned}
$$

Note that when $h$ acts on $\mathcal{M}_{h}$ by 0 what we get is precisely the notion of a coherent Poisson $\mathcal{O}_{X}$-module from above. As the other extreme, assume $\mathcal{M}_{h}$ is $\mathbb{C}[[h]]$-flat. Here (1) allows to recover $\{\cdot, \cdot\}$ from the bimodule structure on $\mathcal{M}_{h}$. Note that the $h$-adic filtration on $\mathcal{M}_{h}$ is automatically complete and separated, this is true for all coherent $\mathcal{D}_{h}$-modules.

When $\mathbb{C}^{\times}$acts on $\mathcal{D}_{h}$ as above, we can talk about graded coherent Poisson $\mathcal{D}_{h}$-bimodules.

We now proceed to $\mathrm{HC}$ bimodules. Let $X:=\operatorname{Spec}(A)$. Let $\mathcal{D}_{h}$ be a graded formal quantization of $X$ and let $\mathcal{A}_{h}$ be the $\mathbb{C}^{\times}$-finite part of $H^{0}\left(X, \mathcal{D}_{h}\right)$. Then $\mathcal{A}:=\mathcal{A}_{h} /(h-1)$ is a filtered quantization of $A$. Set $\hbar:=\sqrt[d]{h}$. We can form the Rees algebra $R_{\hbar}(\mathcal{A})$. Then $R_{\hbar}(\mathcal{A})$ is naturally identified with $\mathbb{C}[\hbar] \otimes_{\mathbb{C}[h]} \mathcal{A}_{h}$. Now let $\mathcal{B}$ be a $\mathrm{HC} \mathcal{A}$-bimodule. Choose a good filtration on $\mathcal{B}$. Then the Rees bimodule $R_{\hbar}(\mathcal{B})$ is a $R_{\hbar}(\mathcal{A})$-bimodule and also a Poisson $\mathcal{A}_{h}$-bimodule. We will call such bimodules graded Poisson $R_{\hbar}(\mathcal{A})$-bimodules.

Let us introduce some notation for HC bimodules in the case when $\mathcal{A}$ is a quantization of a conical symplectic singularity $Y$. Denote the category of $\mathrm{HC} \mathcal{A}$-bimodules by $\mathrm{HC}(\mathcal{A})$.

Let $\mathcal{B} \in \mathrm{HC}(\mathcal{A})$. It is a classical fact that the support of the $\mathbb{C}[Y]$-module gr $\mathcal{B}$ in $Y$ is independent of the choice of a good filtration. This support is called the associated variety of $\mathcal{B}$ and is denoted by $\operatorname{VA}(\mathcal{B})$. This is a Poisson subvariety.

The following lemma is also standard.

Lemma 2.13. Let $\mathcal{B}, \mathcal{B}^{\prime} \in \operatorname{HC}(\mathcal{A})$. Then $\mathcal{B} \otimes_{\mathcal{A}} \mathcal{B}^{\prime}, \operatorname{Hom}_{\mathcal{A}}\left(\mathcal{B}, \mathcal{B}^{\prime}\right), \operatorname{Hom}_{\mathcal{A}^{o p p}}\left(\mathcal{B}, \mathcal{B}^{\prime}\right) \in \operatorname{HC}(\mathcal{A})$ and the associated varieties of these bimodules are contained in $\operatorname{VA}(\mathcal{B}) \cap \mathrm{VA}\left(\mathcal{B}^{\prime}\right)$.

It follows, in particular, that $\operatorname{VA}(\mathcal{B}) \neq Y$ if and only if $\mathcal{B}$ is not faithful as a left (equivalently, right) bimodule. Let us write $\overline{\mathrm{HC}}(\mathcal{A})$ for the Serre quotient

$$
\mathrm{HC}(\mathcal{A}) /\{\mathcal{B} \in \mathrm{HC}(\mathcal{A}) \mid \operatorname{VA}(\mathcal{B}) \neq Y\} .
$$

We call $\overline{\mathrm{HC}}(\mathcal{A})$ the category of $\mathrm{HC}$ bimodules with full support. By Lemma 2.13, this is a rigid monoidal category.

We now turn to a connection between the categories of $\mathrm{HC}$ bimodules and of the graded Poisson $R_{\hbar}(\mathcal{A})$-bimodules. The functor $\mathcal{B}_{\hbar} \rightarrow \mathcal{B}_{\hbar} /(\hbar-1) \mathcal{B}_{\hbar}$ maps from the category of graded Poisson bimodules to the category of $\mathrm{HC}$ bimodules. It is not difficult to see that it is a Serre quotient functor, the kernel consists of the $\hbar$-torsion modules.

The connection described in the previous paragraph can be extended to non-affine varieties. Namely, take a graded formal quantization $\mathcal{D}_{h}$ of a normal variety $X$. We can form the microlocal sheaves $\mathcal{D}_{h, \text { fin }}$ of $\mathbb{C}^{\times}$-finite sections of $\mathcal{D}_{h}$ and $\mathcal{D}:=\mathcal{D}_{h, \text { fin }} /(\hbar-1) \mathcal{D}_{h, \text { fin }}$ on $X$ (where "microlocal" means that the sections are only defined on the $\mathbb{C}^{\times}$-stable open subsets). We can define the notion of a $\mathrm{HC}$ bimodule over $\mathcal{D}$ as a bimodule that has a complete and separated good filtration. The category of $\mathrm{HC} \mathcal{D}$-bimodules is the quotient of the category of graded coherent Poisson $\mathcal{D}_{\hbar}$-bimodules by the full subcategory of $\hbar$ torsion bimodules.

Finally, we need to recall the construction of restriction functors for $\mathrm{HC}$ bimodules considered in this (and greater) generality in [L7, Section 3.3]. We use the setting of Lemma 2.10. Note that both algebras $R_{\hbar}\left(\mathcal{A}_{\lambda}\right), R_{\hbar}\left(\mathbb{A} \otimes \underline{\mathcal{A}}_{\lambda_{i}}\right)$ come equipped with the 
Euler derivations coming from the gradings. We will denote these derivations by eu, eu'. The following claim was obtained in the proof of [L7, Lemma 3.3].

Lemma 2.14. Under the isomorphism of Lemma 2.10, the derivations eu and eu' differ by a derivation of the form $\frac{1}{h} \operatorname{ad}(a)$ for $a \in \mathcal{A}_{\lambda h}^{\wedge y_{i}}$.

Now we recall the construction of a functor $\operatorname{HC}\left(\mathcal{A}_{\lambda}\right) \rightarrow \operatorname{HC}\left(\underline{\mathcal{A}}_{\lambda_{i}}\right)$ that we will denote by $\bullet_{+, i}$ (see [L7, Section 3.3]). Consider the completion $R_{\hbar}^{\wedge}(\mathcal{B})$ at $y_{i}$, this is an $R_{\hbar}^{\wedge}\left(\mathcal{A}_{\lambda}\right)$ bimodule. It comes equipped with the derivation eu that is compatible with the eponymous derivation of $R_{\hbar}^{\wedge}\left(\mathcal{A}_{\lambda}\right)$. By Lemma $\left[2.10, R_{\hbar}^{\wedge}(\mathcal{B})\right.$ can be viewed as an $R_{\hbar}^{\wedge}\left(\mathbb{A} \otimes \underline{\mathcal{A}}_{\lambda_{i}}\right)$ bimodule. We define an operator eu' on $R_{\hbar}^{\wedge}(\mathcal{B})$ as follows: eu' $=$ eu $+\frac{1}{h}$ ad $a$, where $a$ is as in Lemma 2.14. Note that eu' is compatible with the derivation eu' of the algebra $R_{\hbar}\left(\mathbb{A} \otimes \underline{\mathcal{A}}_{\lambda^{i}}\right)$.

It was shown in [L7, Section 3.3] that $R_{\hbar}^{\wedge}(\mathcal{B})$ splits as $R_{\hbar}^{\wedge}(\mathbb{A}) \widehat{\otimes}_{\mathbb{C}[[\hbar]]} \underline{\mathcal{B}}_{\hbar}$, where $\underline{\mathcal{B}}_{\hbar}$ is the centralizer of $R_{\hbar}^{\wedge}(\mathbb{A})$ in $R_{\hbar}^{\wedge}(\mathcal{B})$. In particular, eu' preserves $\underline{\mathcal{B}}_{\hbar}$. Consider the subspace $\underline{\mathcal{B}}_{\hbar, \text { fin }}$ of all eu'-finite elements. This is a $R_{\hbar}\left(\underline{\mathcal{A}}_{\lambda_{i}}\right)$-sub-bimodule. Set $\mathcal{B}_{\dagger, i}:=\underline{\mathcal{B}}_{\hbar, \text { fin }} /(\hbar-1)$.

It was shown in [L1, Sections 3.3,3.4], that this construction indeed gives a functor $\mathrm{HC}\left(\mathcal{A}_{\lambda}\right) \rightarrow \mathrm{HC}\left(\underline{\mathcal{A}}_{\lambda_{i}}\right)$. This functor is exact and tensor. On the level of associated graded bimodules the functor becomes (the algebraization of) the restriction of the Poisson bimodule to the slice. In particular, it descends to $\overline{\mathrm{HC}}\left(\mathcal{A}_{\lambda}\right) \rightarrow \overline{\mathrm{HC}}\left(\underline{\mathcal{A}}_{\lambda_{i}}\right)$.

2.5. Pushforwards of coherent $\mathcal{D}_{h}$-modules. Let $X$ be a normal Poisson variety and $\mathcal{D}_{h}$ be its formal quantization. Recall that a $\mathcal{D}_{h}$-module $M_{h}$ is called coherent if there is an open affine cover $X=\bigcup U_{i}$ such that $\left.M_{h}\right|_{U_{i}}$ is obtained by microlocalizing a finitely generated $H^{0}\left(U_{i}, \mathcal{D}_{h}\right)$-module. In this case, for every open affine subvariety $U \subset X$, the restriction $\left.M_{h}\right|_{U}$ is the microlocalization of $H^{0}\left(U, M_{h}\right)$. Note that every coherent $\mathcal{D}_{h}$-module is automatically complete and separated in the $h$-adic topology.

Now let $\iota: X^{0} \hookrightarrow X$ be an open embedding and let $M_{h}^{0}$ be a coherent $\mathcal{D}_{h}^{0}:=\left.\mathcal{D}_{h}\right|_{X^{0-}}$ module. We set $M_{h, k}^{0}:=M_{h}^{0} / h^{k} M_{h}^{0}$. When $k=1$, we write $M^{0}$ instead of $M_{h, 1}^{0}$. We will assume the following condition:

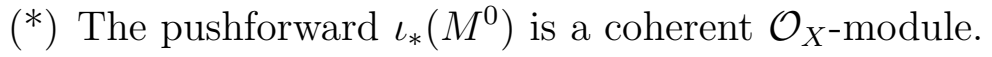

We want to get a sufficient condition for $\iota_{*} M_{h}^{0}$ to be coherent. We note that for a $\mathcal{D}_{h}^{0} /\left(h^{k}\right)$-modules it makes sense to speak about quasi-coherent modules, and the pushforward maps quasi-coherent modules to quasi-coherent ones - for the same reason as for the quasi-coherent $\mathcal{O}_{X}$-modules. If $\left(^{*}\right)$ holds, then $\iota_{*}\left(M_{h, k}^{0}\right)$ is coherent for all $k$. However, $\iota_{*}\left(M_{h}^{0}\right)$ may fail to be coherent: the problem is that, for some open affine subset $U \subset X$, the $H^{0}\left(U, \mathcal{D}_{h}\right)$-module $H^{0}\left(U \cap X^{0}, M_{h}^{0}\right)$ may not be large enough (in fact, it can be zero even if $M_{h}^{0}$ is nonzero).

Let $U \subset X$ be an open affine subvariety. Set $U^{0}:=U \cap X^{0}$. We write $H^{0}\left(U^{0}, M^{0}\right)_{k}$ for the image of $H^{0}\left(U^{0}, M_{h, k}^{0}\right)$ in $H^{0}\left(U^{0}, M^{0}\right)$. Note that the submodules $H^{0}\left(U, M^{0}\right)_{k}$ form a decreasing sequence.

The following lemma gives a sufficient condition for $\iota_{*} M_{h}^{0}$ to be coherent.

Lemma 2.15. Suppose $\left(^{*}\right)$ holds. Let $X=\bigcup_{i=1}^{\ell} U_{i}$ be an open affine cover. Suppose that, for every $i$, the sequence $H^{0}\left(U_{i}^{0}, M^{0}\right)_{k}$ stabilizes. Then $\iota_{*} M_{h}^{0}$ is coherent.

Proof. Note that the condition that the sequence $H^{0}\left(U_{i}^{0}, M^{0}\right)_{k}$ stabilizes is equivalent to the Mittag-Leffler (ML) condition for the inverse system of $H^{0}\left(U_{i}, \mathcal{D}_{h}\right)$-modules $H^{0}\left(U_{i}^{0}, M_{h, k}^{0}\right)$. Since $M_{h}^{0}=\lim _{\longleftarrow} M_{h, k}^{0}$ we see that $H^{0}\left(U_{i}^{0}, M_{h}^{0}\right)=\lim _{\longleftarrow} H^{0}\left(U_{i}^{0}, M_{h, k}^{0}\right)$. Note that, thanks 
to $\left.{ }^{*}\right), H^{0}\left(U_{i}^{0}, M_{h}^{0}\right)$ is a finitely generated $H^{0}\left(U_{i}, \mathcal{D}_{h}\right)$-module. It remains to show that $\left.\left(\iota_{*} M_{h}^{0}\right)\right|_{U_{i}}$ is obtained by microlocalizing $H^{0}\left(U_{i}^{0}, M_{h}^{0}\right)$. This is equivalent to the condition that, for every $f \in \mathbb{C}\left[U_{i}\right]$, the module $H^{0}\left(U_{i, f}^{0}, M_{h}^{0}\right)$ is the microlocalization of $H^{0}\left(U_{i}^{0}, M_{h}^{0}\right)$ at $f$ to be denoted by $H^{0}\left(U_{i}^{0}, M_{h}^{0}\right)\left[f^{-1}\right]$. This condition holds if we replace $M_{h}^{0}$ with $M_{h, k}^{0}$. Then, by ML, it holds for $M_{h}^{0}$.

2.6. Etale lifts of Poisson bimodules. Let $\mathcal{D}_{h}$ be a formal quantization of a smooth symplectic variety $X$. The goal of this section is to make sense of pullbacks of Poisson $\mathcal{D}_{h}$-modules under étale morphisms of symplectic varieties. We will do so by considering jet bundles for Poisson bimodules. Recall that the jet bundles of $\mathcal{O}_{X}$ and of its formal quantization were discussed in Section 2.1.

We can define the notion of a coherent Poisson $\mathrm{J}^{\infty} \mathcal{D}_{h}$-bimodule $\mathfrak{B}_{h}$. By definition, this is a pro-coherent sheaf of $\mathcal{O}_{X}$-modules that comes with

(1) a $\mathrm{J}^{\infty} \mathcal{D}_{h^{-}}$-bimodule structure making it into a locally finitely generated $\mathrm{J}^{\infty} \mathcal{D}_{h^{-}}$ module,

(2) a $\mathbb{C}[[h]]$-linear flat connection $\nabla$ that is compatible with the flat connection on $\mathrm{J}^{\infty} \mathcal{D}_{h}$

(3) and a flat bracket map $\{\cdot, \cdot\}: \mathrm{J}^{\infty} \mathcal{D}_{h} \otimes_{\mathbb{C}} \mathfrak{B}_{h} \rightarrow \mathfrak{B}_{h}$ satisfying the axioms (1)-(3) listed in Section 2.4,

(4) such that $\mathfrak{B}_{h}$ is complete and separated in the $h$-adic topology and, moreover, for each $k \in \mathbb{Z}_{>0}$, the quotient $h^{k-1} \mathfrak{B}_{h} / h^{k} \mathfrak{B}_{h}$ is the jet bundle of a coherent Poisson $\mathcal{O}_{X}$-module.

Now let $\mathcal{B}_{h}$ be a Poisson $\mathcal{D}_{h}$-bimodule. Similarly to what was done in Section 2.1, we can form the jet bundle $\mathrm{J}^{\infty} \mathcal{B}_{h}$, which is a pro-coherent sheaf on $X$ with a flat connection. It is easy to see that this is a coherent Poisson $\mathrm{J}^{\infty} \mathcal{D}_{h}$-bimodule.

Lemma 2.16. The functors $\mathcal{B}_{h} \mapsto \mathrm{J}^{\infty} \mathcal{B}_{h}$ and $\mathfrak{B}_{h} \mapsto \mathfrak{B}_{h}^{\nabla}$ are equivalences between the category of coherent Poisson bimodules over $\mathcal{D}_{h}$ and the category of coherent Poisson $\mathrm{J}^{\infty} \mathcal{D}_{h}$-bimodules.

Proof. We write $\bullet \nabla$ for the functor of taking flat sections. By the construction, $\left(\mathrm{J}^{\infty} \mathcal{B}_{h}\right)^{\nabla}=$ $\mathcal{B}_{h}$. On the other hand let $\mathfrak{B}_{h}$ be a coherent Poisson $\mathrm{J}^{\infty} \mathcal{D}_{h}$-module. Note that $\mathfrak{B}_{h}^{\nabla}$ is complete and separated in the $h$-adic topology and $\mathfrak{B}_{h}^{\nabla} / h \mathfrak{B}_{h}^{\nabla} \hookrightarrow\left(\mathfrak{B}_{h} / h \mathfrak{B}_{h}\right)^{\nabla}$. So $\mathfrak{B}_{h}^{\nabla}$ is a coherent Poisson $\mathcal{D}_{h}$-bimodule.

Now we need to show that the jet bundle $\mathrm{J}^{\infty}\left(\mathfrak{B}_{h}^{\nabla}\right)$ is functorially isomorphic to $\mathfrak{B}_{h}$. First of all, observe that the left $\mathrm{J}^{\infty} \mathcal{D}_{h}$-action on $\mathrm{J}^{\infty}\left(\mathcal{B}_{h}\right)$ for any $\mathcal{B}_{h}$ induces an isomorphism $\mathrm{J}^{\infty} \mathcal{D}_{h} \widehat{\otimes}_{\mathcal{D}_{h}} \mathcal{B}_{h} \stackrel{\sim}{\rightarrow} \mathrm{J}^{\infty} \mathcal{B}_{h}$. This gives rise to a Poisson bimodule homomorphism $\mathrm{J}^{\infty}\left(\mathfrak{B}_{h}^{\nabla}\right) \rightarrow$ $\mathfrak{B}_{h}$. We want to show that it is an isomorphism.

We start by showing that, for any vector bundle $\mathcal{V}$ on $X$, we have

$$
R^{1}\left(\mathrm{~J}^{\infty} \mathcal{V}\right)^{\nabla}=0
$$

This will follow from a stronger statement: $R \mathcal{H} \operatorname{Hom}_{D_{X}}\left(\mathcal{O}_{X}, \mathrm{~J}^{\infty} \mathcal{V}\right)=\mathcal{V}$ for all vector bundles $\mathcal{V}$. The latter equality is standard.

Thanks to (2.1), for all $k$, we have

$$
\begin{aligned}
& \mathfrak{B}_{h}^{\nabla} / h^{k} \mathfrak{B}_{h}^{\nabla} \stackrel{\sim}{\rightarrow}\left(\mathfrak{B}_{h} / h^{k} \mathfrak{B}_{h}\right)^{\nabla}, \\
& \mathrm{J}^{\infty}\left(\left(\mathfrak{B}_{h} / h^{k} \mathfrak{B}_{h}\right)^{\nabla}\right) \stackrel{\sim}{\rightarrow} \mathfrak{B}_{h} / h^{k} \mathfrak{B}_{h} .
\end{aligned}
$$

Since $\mathfrak{B}_{h}$ is complete and separated in the $h$-adic topology, we deduce that $\mathrm{J}^{\infty}\left(\mathfrak{B}_{h}^{\nabla}\right) \stackrel{\sim}{\rightarrow} \mathfrak{B}_{h}$. 
Lemma 2.16 allows to define the pullback of Poisson bimodules under an étale morphism intertwining symplectic forms. Namely, let $\varphi: X^{1} \rightarrow X^{2}$ be such a morphism. Let $\mathcal{D}_{h}^{1}$ be a formal quantization of $X^{1}$. Then $\varphi^{*} \mathrm{~J}^{\infty} \mathcal{D}_{h}^{1}$ is a quantum jet bundle on $X^{2}$. Passing to the sheaf of flat sections, we get a formal quantization $\mathcal{D}_{h}^{2}$ of $X^{2}$ with $\mathrm{J}^{\infty} \mathcal{D}_{h}^{2}=\varphi^{*} \mathrm{~J}^{\infty} \mathcal{D}_{h}^{1}$.

Now let $\mathcal{B}_{h}^{1}$ be a Poisson $\mathcal{D}_{h}^{1}$-bimodule. Then $\varphi^{*}\left(\mathrm{~J}^{\infty} \mathcal{B}_{h}^{1}\right)$ is a coherent Poisson $\mathrm{J}^{\infty} \mathcal{D}_{h^{-}}$ module. We set $\varphi^{*} \mathcal{B}_{h}^{1}:=\left(\varphi^{*} \mathrm{~J}^{\infty} \mathcal{B}_{h}^{1}\right)^{\nabla}$. The following properties are straightforward from the construction.

Lemma 2.17. The following claims hold:

- If $\mathcal{B}_{h}^{1}$ is annihilated by $h$ (so that $\mathcal{B}_{h}^{1}$ is a vector bundle with a flat connection), then $\varphi^{*} \mathcal{B}_{h}^{1}$ is the usual pull-back of a vector bundle with a flat connection.

- The functor $\varphi^{*}$ is exact, faithful and $\mathbb{C}[[h]]$-linear.

- We have a natural isomorphism of left $\mathcal{D}_{h}^{2}$-modules

$$
\varphi^{*} \mathcal{B}_{h}^{1} \cong \mathcal{D}_{h}^{2} \widehat{\otimes}_{\varphi^{-1}} \mathcal{D}_{h}^{1} \varphi^{-1} \mathcal{B}_{h}^{1} .
$$

\section{Extending Poisson Bimodules from $Y^{\text {reg }}$ To $Y$}

3.1. Main result. Let $Y$ be a conical symplectic singularity and $\mathcal{A}$ its filtered quantization.

Let $\mathcal{D}_{\hbar}$ be the microlocalization of $R_{\hbar}(\mathcal{A})$ to $Y$ so that $\mathcal{D}_{\hbar}=\mathbb{C}[\hbar] \otimes_{\mathbb{C}[h]} \mathcal{D}_{h}$, where $\mathcal{D}_{h}$ is the microlocalization of $\mathcal{A}_{\lambda h}$ and $\hbar^{d}=h$. Set $\mathcal{A}_{\hbar}:=\Gamma\left(\mathcal{D}_{\hbar}\right)$, this is the $\hbar$-adic completion of $R_{\hbar}(\mathcal{A})$. We write $\mathcal{D}_{\hbar}^{\text {reg }}$ for the restriction of $\mathcal{D}_{\hbar}$ to $Y^{\text {reg }}$. Recall that we also consider the open subvariety $Y^{\text {sreg }} \subset Y$, defined by $Y^{\text {sreg }}:=Y^{\text {reg }} \sqcup \bigsqcup_{i=1}^{k} \mathcal{L}_{i}$, where $\mathcal{L}_{1}, \ldots, \mathcal{L}_{k}$ are all codimension 2 symplectic leaves. We consider the restriction $\mathcal{D}_{\hbar}^{\text {sreg }}$. Also set $Y_{i}:=\operatorname{Spec}\left(\mathbb{C}[Y]^{\wedge_{y}}\right)$, where $y \in \mathcal{L}_{i}$ and $\mathbb{C}[Y]^{\wedge_{y}}$ denote the completion of $\mathbb{C}[Y]$ at $y$. We write $Y_{i}^{\times}$for $Y_{i} \backslash \mathcal{L}_{i}$. We can restrict $\mathcal{D}_{\hbar}^{\text {sreg }}$ to $Y_{i}$ getting a formal quantization $\left.\mathcal{D}_{\hbar}\right|_{Y_{i}}$. We can further restrict this formal quantization to $Y_{i}^{\times}$.

Our primary goal in this section is to understand conditions for a coherent Poisson $\mathcal{D}_{\hbar}^{\text {reg }}$-bimodule $\mathcal{B}_{\hbar}$ that is flat over $\mathbb{C}[[\hbar]]$ (the only case we are interested in) to extend to a graded Poisson $\mathcal{A}_{\hbar}$-bimodule. Note that when $\hbar$ acts on $\mathcal{B}_{\hbar}$ by zero, then we can take $H^{0}\left(Y^{r e g}, \mathcal{B}_{\hbar}\right)$ for this extension, indeed, $\mathcal{B}_{\hbar}$ is a vector bundle and since $\operatorname{codim}_{Y} Y^{\operatorname{sing}} \geqslant 2$, the global sections of every vector bundle on $Y^{\text {reg }}$ is a finitely generated $\mathbb{C}[Y]$-module.

Here is the main result to be proved in this section.

Proposition 3.1. Let $\mathcal{B}_{\hbar}$ be $\mathbb{C}[[\hbar]]$-flat. Then the following two conditions are equivalent:

(1) The restriction of $\Gamma\left(\mathcal{B}_{\hbar}\right)$ to $Y^{\text {reg }}$ coincides with $\mathcal{B}_{\hbar}$.

(2) The restriction of $\Gamma\left(\left.\mathcal{B}_{\hbar}\right|_{Y_{i}^{\times}}\right)$to $Y_{i}^{\times}$coincides with $\left.\mathcal{B}_{\hbar}\right|_{Y_{i}^{\times}}$for all $i=1, \ldots, k$.

We will explain the meaning of $\left.\mathcal{B}_{\hbar}\right|_{Y_{i}^{\times}}$below in Section 3.2. Note that $(1) \Rightarrow(2)$ is relatively easy, while $(2) \Rightarrow(1)$ is harder.

The proof is in two steps. First, let $\iota$ denote the inclusion $Y^{r e g} \hookrightarrow Y^{\text {sreg }}$. So we get a Poisson $\mathcal{D}_{\hbar}^{\text {sreg }}$-bimodule $\iota_{*} \mathcal{B}_{\hbar}$ that is flat over $\mathbb{C}[[\hbar]]$. In Section $[3.3$, we will show that condition (2) of the proposition is equivalent to the claim that $\iota_{*} \mathcal{B}_{\hbar}$ is coherent. Then in Section 3.4 we show that, if $(2)$ holds, then $\left.\Gamma\left(\mathcal{B}_{\hbar}\right)\right|_{Y^{r e g}} \cong \iota^{*} \iota_{*} \mathcal{B}_{\hbar}$. This will imply Proposition 3.1. 
3.2. Construction of $\left.\mathcal{B}_{\hbar}\right|_{Y_{i}^{\times}}$. The goal of this section is to make sense of the Poisson bimodule $\left.\mathcal{B}_{\hbar}\right|_{Y_{i}^{\times}}$and study properties of this Poisson bimodule. Let $\varphi$ denote the morphism $Y_{i}^{\times} \rightarrow Y^{\text {reg }}$ induced by the inclusion $Y_{i} \rightarrow Y$. We define $\left.\mathcal{B}_{\hbar}\right|_{Y_{i}^{\times}}$as

$$
\left.\mathcal{D}_{\hbar}\right|_{Y_{i}^{\times}} \otimes_{\varphi^{-1}} \mathcal{D}_{\hbar} \varphi^{-1} \mathcal{B}_{\hbar} .
$$

This is a coherent $\left.\mathcal{D}_{\hbar}\right|_{Y_{i} \times}$-module. What we need to do is to construct the bracket map satisfying conditions (2) and (3) from Section 2.4.

Let $U$ be an open affine neighborhood in $X$ of the closed point $y$ in $Y_{i}$. Note that we have the bracket map $\left.\left.\mathcal{D}_{\hbar}(U) \otimes_{\mathbb{C}[\hbar]]} \mathcal{B}_{\hbar}\right|_{Y_{i}^{\times}} \rightarrow \mathcal{B}_{\hbar}\right|_{Y_{i}^{\times}}$with required properties.

Lemma 3.2. This bracket extends to a bracket $\left.\left.\left.\mathcal{D}_{\hbar}\right|_{Y_{i}^{\times}} \otimes_{\mathbb{C}[\hbar \hbar]]} \mathcal{B}_{\hbar}\right|_{Y_{i}^{\times}} \rightarrow \mathcal{B}_{\hbar}\right|_{Y_{i}^{\times}}$satisfying (2) and (3).

Proof. Consider the case when $\hbar^{k}$ annihilates $\mathcal{B}_{\hbar}$ for some $k>0$. Then we can consider the push-forward $\overline{\mathcal{B}}_{\hbar}$ of $\mathcal{B}_{\hbar}$ to $Y$, this is a coherent Poisson $\mathcal{D}_{\hbar}$-bimodule. Consider the restriction $\overline{\mathcal{B}}_{\hbar}^{\wedge y}$ to $Y_{i}$. It comes with the bracket with $\mathcal{D}_{\hbar}^{\wedge y}$. Then we can localize the bracket to $Y_{i}^{\times}$. This settles the case when $\mathcal{B}_{\hbar}$ is annihilated by $\hbar^{k}$. To handle the general case we notice that the bracket is continuous in the $\hbar$-adic topology and $\left.\mathcal{B}_{\hbar}\right|_{Y_{i}^{\times}}=\left.\lim _{\longleftarrow}\left(\mathcal{B}_{\hbar} / \hbar^{k} \mathcal{B}_{\hbar}\right)\right|_{Y_{i}^{\times}}$.

The following properties are straightforward from the construction.

Lemma 3.3. The following claims hold:

- If $\mathcal{B}_{\hbar}$ is annihilated by $\hbar$ (so that $\mathcal{B}_{\hbar}$ is a vector bundle with a flat connection), then $\left.\mathcal{B}_{\hbar}^{1}\right|_{Y_{i}^{\times}}$is the usual pull-back of a vector bundle with a flat connection.

- The functor $\left.\bullet\right|_{Y_{i}^{\times}}$is exact, faithful and $\mathbb{C}[[\hbar]]$-linear. In particular, $\left.\mathcal{B}_{\hbar}\right|_{Y_{i}^{\times}}=$ $\left.\lim _{\longleftarrow}\left(\mathcal{B}_{\hbar} / \hbar^{k} \mathcal{B}_{\hbar}\right)\right|_{Y_{i}^{\times}}$.

3.3. Extension to codimension 2. The goal of this section is to prove the following lemma.

Lemma 3.4. Let $\iota: Y^{r e g} \hookrightarrow Y^{\text {sreg }}$ denote the inclusion. Then the following two conditions are equivalent:

(1) $\iota_{*} \mathcal{B}_{\hbar}$ is coherent.

(2) The restriction of $H^{0}\left(Y_{i}^{\times},\left.\mathcal{B}_{\hbar}\right|_{Y_{i}^{\times}}\right)$to $Y_{i}^{\times}$coincides with $\left.\mathcal{B}_{\hbar}\right|_{Y_{i}^{\times}}$for all $i=1, \ldots, k$.

Proof. Let us prove $(1) \Rightarrow(2)$. Note that $\left.\left.H^{0}\left(Y_{i}^{\times},\left.\mathcal{B}_{\hbar}\right|_{Y_{i}^{\times}}\right)\right|_{Y_{i}^{\times}} \hookrightarrow \mathcal{B}_{\hbar}\right|_{Y_{i}^{\times}}$. So we need to prove this map is surjective. Consider the restriction $\left(\iota_{*} \mathcal{B}_{\hbar}\right)^{\wedge_{y}}$ of $\iota_{*} \mathcal{B}_{\hbar}$ to $Y_{i}$. Then $\left(\iota_{*} \mathcal{B}_{\hbar}\right)^{\wedge_{y}} \hookrightarrow$ $H^{0}\left(Y_{i}^{\times},\left.\mathcal{B}_{\hbar}\right|_{Y_{i}^{\times}}\right)$. On the other hand, $\iota^{*} \iota_{*} \mathcal{B}_{\hbar} \cong \mathcal{B}_{\hbar}$ and hence $\left.\left.\left(\iota_{*} \mathcal{B}_{\hbar}\right)^{\wedge y}\right|_{Y_{i}^{\times}} \stackrel{\sim}{\rightarrow} \mathcal{B}_{\hbar}\right|_{Y_{i}^{\times}}$. This implies (2).

Now we prove $(2) \Rightarrow(1)$. We can find an open affine cover $Y^{\text {sreg }}=\bigcup_{j=1}^{\ell} U_{i}$ such that each $U_{j}$ intersects at most one codimension 2 symplectic leaf. We will check the condition of Lemma 2.15 for $M_{h}^{0}=\mathcal{B}_{\hbar}$ and this cover, this will imply (1). Let $U=U_{j}$ for some $j$. So we need to prove that the sequence $H^{0}\left(U^{r e g}, M^{0}\right)_{m}$ stabilizes. If $U \subset Y^{r e g}$, there is nothing to prove. So let $\mathcal{L}:=U^{\text {sing }}$, it is an open subvariety in some $\mathcal{L}_{i}$. Note that each $H^{0}\left(U^{r e g}, M^{0}\right)_{m}$ is a Poisson $\mathbb{C}[U]$-submodule in the finitely generated Poisson $\mathbb{C}[U]$ module $H^{0}\left(U^{\text {reg }}, M^{0}\right)$. Since $H^{0}\left(U^{\text {reg }}, M^{0}\right) / H^{0}\left(U^{\text {reg }}, M^{0}\right)_{m}$ is supported on $\mathcal{L}$, we see that $H^{0}\left(U^{r e g}, M^{0}\right) / H^{0}\left(U^{r e g}, M^{0}\right)_{m}$ admits a finite filtration by vector bundles on $\mathcal{L}$. In 
particular, it is enough to show that, for $y \in \mathcal{L}$, the sequence $\left[H^{0}\left(U^{r e g}, M^{0}\right)_{m}\right]^{\wedge_{y}}$ stabilizes. This is done in three steps. We will use the notation of Lemma 2.15,

Step 1. We claim that $H^{n}\left(U^{r e g}, M^{0}\right)^{\wedge_{y}} \stackrel{\sim}{\rightarrow} H^{n}\left(Y_{i}^{\times},\left.M^{0}\right|_{Y_{i}^{\times}}\right)$for all $n$. Indeed, we cover $U^{r e g}$ with principal open subsets $V_{s}=U_{f_{s}}$ for a finite collection $f_{s} \in \mathbb{C}[U]$. Let $M:=$ $H^{0}\left(U^{r e g}, M^{0}\right)$. Then $H^{n}\left(U^{r e g}, M^{0}\right)$ is the cohomology of the Cech complex for $M$ and the cover $V_{s}$ of $U^{r e g}$. Since the completion functor is exact, we see that it sends to Cech complex for $M$ to that of $M^{\wedge_{y}}$. But $H^{n}\left(Y_{i}^{\times},\left.M^{0}\right|_{Y_{i}^{\times}}\right)$is the cohomology space for the Cech complex of $M^{\wedge_{y}}$. Our isomorphism is proved.

Step 2. We claim that $H^{n}\left(U^{r e g}, M_{\hbar, p}^{0}\right)^{\wedge} \stackrel{\sim}{\rightarrow} H^{n}\left(Y_{i}^{\times},\left.M_{\hbar, p}^{0}\right|_{Y_{i}^{\times}}\right)$for all $n$ and $p$. This follows by induction on $p$ using Step 1 and the 5-lemma.

Step 3. Thanks to Step 2, we will be done if we know that the sequence $H^{0}\left(Y_{i}^{\times},\left.M^{0}\right|_{Y_{i}^{\times}}\right)_{m}$ stabilizes. Note that $H^{0}\left(Y_{i}^{\times},\left.\mathcal{B}_{\hbar}\right|_{Y_{i}^{\times}}\right) / \hbar H^{0}\left(Y_{i}^{\times},\left.\mathcal{B}_{\hbar}\right|_{Y_{i}^{\times}}\right) \hookrightarrow H^{0}\left(Y_{i}^{\times},\left.M^{0}\right|_{Y_{i}^{\times}}\right)$. The image is contained in all $H^{0}\left(Y_{i}^{\times},\left.M^{0}\right|_{Y_{i}^{\times}}\right)_{m}$. Thanks to $(2)$, the cokernel is supported on $\mathcal{L}^{\wedge y}$ and hence has finite length. So the sequence $H^{0}\left(Y_{i}^{\times},\left.M^{0}\right|_{Y_{i}^{\times}}\right)_{m}$ indeed stabilizes. This completes the proof.

3.4. Extension to $Y$. Let $\iota^{\prime}$ denote the embedding of $Y^{\text {sreg }}$ to $Y$. The goal of this section is to prove the following result. Together with Lemma 3.4, this will finish the proof of Proposition 3.1.

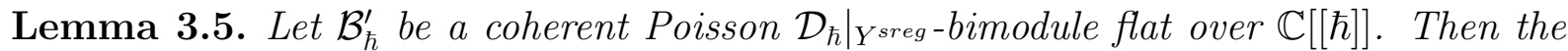
restriction of $\Gamma\left(\mathcal{B}_{\hbar}^{\prime}\right)$ to $Y^{\text {sreg }}$ coincides with $\mathcal{B}_{\hbar}^{\prime}$.

Proof. It is enough to show that

(*) $H^{1}\left(Y^{\text {sreg }}, \mathcal{B}_{\hbar}^{\prime}\right)$ is a finitely generated $\mathcal{A}_{\hbar}$-module supported on $Y \backslash Y^{\text {sreg }}$.

Indeed, if we know $\left(^{*}\right)$, then the restriction of $H^{0}\left(Y^{\text {sreg }}, \mathcal{B}_{\hbar}^{\prime}\right) / \hbar H^{0}\left(Y^{\text {sreg }}, \mathcal{B}_{\hbar}^{\prime}\right)$ to $Y^{\text {sreg }}$ coincides with $\mathcal{B}_{\hbar}^{\prime} / \hbar \mathcal{B}_{\hbar}^{\prime}$. Since $\mathcal{B}_{\hbar}^{\prime}$ is flat over $\mathbb{C}[[\hbar]]$, it follows that $\left.H^{0}\left(Y^{\text {sreg }}, \mathcal{B}_{\hbar}^{\prime}\right)\right|_{Y^{\text {sreg }}} \stackrel{\sim}{\rightarrow} \mathcal{B}_{\hbar}^{\prime}$.

Following the proof of [GL, Lemma 5.6.3], we see that $\left(^{*}\right)$ will follow once we know that $H^{1}\left(Y^{\text {sreg }}, \mathcal{B}_{\hbar}^{\prime} / \hbar \mathcal{B}_{\hbar}^{\prime}\right)$ is a finitely generated $\mathbb{C}[Y]$-module, automatically supported on $Y \backslash Y^{\text {sreg }}$. So we proceed to proving that

(**) $H^{1}\left(Y^{\text {sreg }}, \mathcal{B}_{\hbar}^{\prime} / \hbar \mathcal{B}_{\hbar}^{\prime}\right)$ is finitely generated.

Recall that $\iota$ denotes the embedding of $Y^{\text {reg }}$ into $Y^{\text {sreg }}$. Let $\mathcal{B}_{0}:=\iota_{*} \iota^{*}\left(\mathcal{B}_{\hbar}^{\prime} / \hbar \mathcal{B}_{\hbar}^{\prime}\right)$ so that we have an exact sequence

$$
0 \rightarrow \mathcal{B}_{\hbar}^{\prime} / \hbar \mathcal{B}_{\hbar}^{\prime} \rightarrow \mathcal{B}_{0} \rightarrow \mathcal{V} \rightarrow 0
$$

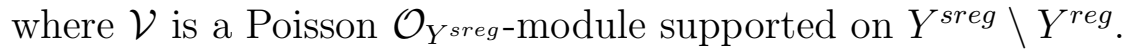

Now consider a part of the long exact sequence induced by (3.1):

$H^{0}\left(Y^{\text {sreg }}, \mathcal{B}_{\hbar}^{\prime} / \hbar \mathcal{B}_{\hbar}^{\prime}\right) \rightarrow H^{0}\left(Y^{\text {sreg }}, \mathcal{B}_{0}\right) \rightarrow H^{0}\left(Y^{\text {sreg }}, \mathcal{V}\right) \rightarrow H^{1}\left(Y^{\text {sreg }}, \mathcal{B}_{\hbar}^{\prime} / \hbar \mathcal{B}_{\hbar}^{\prime}\right) \rightarrow H^{1}\left(Y^{\text {sreg }}, \mathcal{B}_{0}\right)$.

Note that $\mathcal{V}$ is filtered by vector bundles on $Y^{\text {sreg }} \backslash Y^{\text {reg }}$. We have codim $Y^{\text {sing }} Y^{\text {sing }} \backslash$ $Y^{\text {sreg }} \geqslant 2$. It follows that for every vector bundle on $Y^{\text {sreg }} \backslash Y^{\text {reg }}$ its global section is a finitely generated $\mathbb{C}[Y]$-module. From here one deduces that $H^{0}\left(Y^{\text {sreg }}, \mathcal{V}\right)$ is a finitely generated $\mathbb{C}[Y]$-module supported on $Y^{\text {sing }}$. It follows that the cokernel of $H^{0}\left(Y^{\text {sreg }}, \mathcal{B}_{0}\right) \rightarrow$ $H^{0}\left(Y^{\text {sreg }}, \mathcal{V}\right)$ is finitely generated over $\mathbb{C}[Y]$. So $\left.{ }^{* *}\right)$ reduces to the claim that $H^{1}\left(Y^{\text {sreg }}, \mathcal{B}_{0}\right)$ is finitely generated over $\mathbb{C}[Y]$.

A key step here is to show that $\mathcal{B}_{0}$ is maximal Cohen-Macaulay on $Y^{\text {sreg }}$. Let $\pi$ denote the quotient morphism $\tilde{Y} \rightarrow \tilde{Y} / \Gamma=Y$, where $\Gamma=\pi_{1}^{\text {alg }}\left(Y^{r e g}\right), \tilde{Y}^{0}$ is the universal cover of 
$Y^{\text {reg }}$ and $\tilde{Y}=\operatorname{Spec}\left(\mathbb{C}\left[\tilde{Y}^{0}\right]\right)$. By Lemma $\left[2.12, \iota^{*}\left(\mathcal{B}_{\hbar}^{\prime} / \hbar \mathcal{B}_{\hbar}^{\prime}\right)\right.$ is the direct sum of $\Gamma$-isotypic components in $\pi_{*} \mathcal{O}_{\tilde{Y}^{0}}$. It follows that $\iota_{*}^{\prime} \mathcal{B}_{0}$ is the direct sum of $\Gamma$-isotypic components of $\pi_{*} \mathcal{O}_{\tilde{Y}}$. Lemma 2.5 implies that $\tilde{Y}$ is Cohen-Macaulay. Therefore $\pi_{*} \mathcal{O}_{\tilde{Y}}$ is a maximal Cohen-Macaulay sheaf on $Y$, hence $\mathcal{B}_{0}$ is a maximal Cohen-Macaulay sheaf on $Y^{\text {sreg }}$.

Now we can use [Gr, Expose VIII, Cor. 2.3] (together with the standard exact sequence relating $H^{*}\left(Y^{\text {sreg }}, \bullet\right)$ to $\left.H_{Y \backslash Y^{\text {sreg }}}^{*}(\bullet)\right)$ to see that $H^{1}\left(Y^{\text {sreg }}, \mathcal{B}_{0}\right)$ is finitely generated.

\section{ENHANCED RESTRICTION FUNCTOR}

In this section we partially generalize constructions from [L1, L3] of "enhanced" restriction functors. Namely, we are going to produce a full embedding $\overline{\mathrm{HC}}\left(\mathcal{A}_{\lambda}\right) \hookrightarrow \mathbb{C} \Gamma$-mod of monoidal categories, where $\Gamma=\pi_{1}^{a l g}\left(Y^{r e g}\right)$. This functor upgrades the usual restriction functor from [L7, Section 3.3] associated to the open leaf whose target category is Vect.

The functor we need will be constructed as the compostion of two intermediate functors. The first functor will be a full monoidal embedding $\overline{\mathrm{HC}}\left(\mathcal{A}_{\lambda}\right) \hookrightarrow \mathrm{HC}^{\Gamma}\left(\tilde{\mathcal{A}}_{\lambda^{1}}^{0}\right)$ (the definition of the latter category will be given in Section 4.2). Then we will produce a monoidal equivalence $\mathrm{HC}^{\Gamma}\left(\tilde{\mathcal{A}}_{\lambda^{1}}^{0}\right) \stackrel{\sim}{\rightarrow} \mathbb{C} \Gamma$-mod. In Section 4.4 we establish basic properties of the composite functor $\overline{\mathrm{HC}}\left(\mathcal{A}_{\lambda}\right) \hookrightarrow \mathbb{C} \Gamma$-mod.

The last two sections contain developments that are very closely related to to the enhanced restriction functor. In Section 4.5 we will first give an alternative formulation of the extension criterium from Section 3 in terms of the representations of the groups $\Gamma, \Gamma_{i}, i=1, \ldots, k$. Second, let $\Gamma^{\prime} \subset \Gamma$ be a normal subgroup and $\hat{Y}$ be the cover of $Y$ corresponding to $\Gamma / \Gamma^{\prime}$. Let $\hat{\mathcal{A}}$ be a quantization of $\hat{Y}$ with a $\Gamma / \Gamma^{\prime}$-action. We will relate the categories $\overline{\mathrm{HC}}(\hat{\mathcal{A}})$ and $\overline{\mathrm{HC}}\left(\hat{\mathcal{A}}^{\Gamma / \Gamma^{\prime}}\right)$. These two results play a crucial role in describing $\overline{\mathrm{HC}}\left(\mathcal{A}_{\lambda}\right)$. Finally, in Section 4.6 we discuss translation equivalences between the categories $\overline{\mathrm{HC}}$ for different parameters and show that these equivalences intertwine the enhanced restriction functors.

4.1. Regluing quantizations. In this section we are going to relate quantizations of $Y^{\text {reg }}$ that have the same period lying in $H^{2}\left(Y^{r e g}, \mathbb{C}\right)$. More precisely, we are going to show that any two such graded formal quantizations $\mathcal{D}_{h}^{1}, \mathcal{D}_{h}^{2}$ are obtained from one another by gluing with respect to a 1-cocycle of "almost inner" automorphisms. This is a partial generalization of a regluing result from [L3, Section 2.5].

Let us cover $Y^{\text {reg }}$ with affine open $\mathbb{C}^{\times}$-stable subsets $U_{i}$. Since $\mathcal{D}_{h}^{1}, \mathcal{D}_{h}^{2}$ have the same period and the period is functorial, we see that $\left.\mathcal{D}_{h}^{1}\right|_{U_{i}},\left.\mathcal{D}_{h}^{2}\right|_{U_{i}}$ have the same period. By Corollary [2.3, $\left.\left.\mathcal{D}_{h}^{1}\right|_{U_{i}} \cong \mathcal{D}_{h}^{2}\right|_{U_{i}}$, a $\mathbb{C}^{\times}$-equivariant isomorphism. So $\mathcal{D}_{h}^{2}$ is obtained from $\mathcal{D}_{h}^{1}$ via regluing by a 1-cocycle of automorphisms $\theta_{i j} \in \operatorname{Aut}\left(\left.\mathcal{D}_{h}^{1}\right|_{U_{i} \cap U_{j}}\right)$ such that $\theta_{i j}$ is the identity modulo $h$.

The following claim is the main result of this section.

Proposition 4.1. There are elements $\left.f_{i j} \in \mathcal{D}_{h}^{1}\right|_{U_{i j}}$ that

- are $\mathbb{C}^{\times}$-invariant

- $\theta_{i j}=\exp \left(\operatorname{ad}\left(f_{i j}\right)\right)$ and $f_{i j}=-f_{j i}$ for all $i, j$,

- and $f_{i k}=\log \left(\exp \left(f_{i j}\right) \exp \left(f_{j k}\right)\right)$ for all $i, j, k$.

Proof. Let $\delta_{i j}, \alpha_{i j}$ have the same meaning as in the discussion preceding Lemma 2.1.

Since the periods of $\mathcal{D}_{h}^{1}, \mathcal{D}_{h}^{2}$ coincide, it follows from Lemma 2.1 that $\left(\alpha_{i j}\right)$ is a 1coboundary: there are closed forms $\alpha_{i}$ and functions $\underline{f}_{i j}$ with $\underline{f}_{j i}=-\underline{f}_{i j}, \alpha_{i j}=\alpha_{i}-\alpha_{j}+$ 
$d \underline{f}_{i j}$ and $\underline{f}_{k i}+\underline{f}_{i j}+\underline{f}_{j k}=0$. We can further assume that the forms $\alpha_{i}$ and the functions $\underline{f}_{i j}$ are $\mathbb{C}^{\times}$-invariant.

Let $\underline{\delta}_{i}$ be the symplectic vector field on $U_{i}$ corresponding to $\alpha_{i}$. By Lemma 2.4 , $\underline{\delta}_{i}$ lifts to a derivation $\delta_{i}$ of $\left.\mathcal{D}_{h}^{1}\right|_{U_{i}}$. We can assume that $\delta_{i}$ has degree $-d$ with respect to the $\mathbb{C}^{\times}$-action. So we can replace $\theta_{i j}$ with $\exp \left(-h \delta_{i}\right) \theta_{i j} \exp \left(h \delta_{j}\right)$ and assume that $\alpha_{i}=0$. Hence $\alpha_{i j}=d \underline{f}_{i j}$.

Let us prove that there are $\mathbb{C}^{\times}$-invariant elements $\left.f_{i j} \in D_{h}^{1}\right|_{U_{i j}}$ forming a Čech cocycle such that $\delta_{i j}=h^{-1} \operatorname{ad}\left(f_{i j}\right)$. This is true modulo $h$ by the previous paragraph. Let $\underline{f}_{i j}^{\prime}$ denote an arbitrary $\mathbb{C}^{\times}$-invariant lift of $\underline{f}_{i j}$ to $\left.\mathcal{D}_{h}^{1}\right|_{U_{i j}}$. The derivation $h^{-1}\left(\delta_{i j}-h^{-1} \operatorname{ad}\left(\underline{f}_{i j}^{\prime}\right)\right)$ has degree $-2 d$. The 1-form corresponding to this derivation modulo $h$ therefore has degree $-d$. It is closed an hence exact. Arguing in this way, we see that there are elements $f_{i j}$ with required properties.

It remains to prove that $f_{i k}=\log \left(\exp \left(f_{i j}\right) \exp \left(f_{j k}\right)\right)$. Since $\left(\exp \left(\operatorname{ad}\left(f_{i j}\right)\right)\right)$ is a cocycle, the element

$$
z_{i j k}:=\log \left(\exp \left(f_{k i}\right) \exp \left(f_{i j}\right) \exp \left(f_{j k}\right)\right)
$$

is central in $\left.\mathcal{D}_{h}^{1}\right|_{U_{i j k}}$. So it is a formal power series $g_{i j k}(h)$. Since we know that $\underline{f}_{i j}+\underline{f}_{j k}+$ $\underline{f}_{k i}=0$ we see that $g_{i j k}(h)$ is divisible by $h$. On the other hand, $z_{i j k}$ is $\mathbb{C}^{\times}$-invariant hence constant. So it follows that it is zero and completes the proof of the proposition.

4.2. Embedding $\overline{\mathrm{HC}}\left(\mathcal{A}_{\lambda}\right) \hookrightarrow \mathrm{HC}^{\Gamma}\left(\tilde{\mathcal{A}}_{\lambda^{1}}^{0}\right)$. The goal of this section is to produce a full embedding $\mathrm{HC}\left(\mathcal{A}_{\lambda}\right) \hookrightarrow \mathrm{HC}^{\Gamma}\left(\tilde{\mathcal{A}}_{\lambda^{1}}^{0}\right)$ of monoidal categories. The notation here is as follows. As in Section 2.3, let $\tilde{Y}^{0}$ denote the covering of $Y^{\text {reg }}$ with Galois group $\Gamma$, this is an open subset in the conical symplectic singularity $\tilde{Y}$. Note that we have an embedding $H^{2}\left(Y^{r e g}, \mathbb{C}\right)=H^{2}\left(\tilde{Y}^{0}, \mathbb{C}\right)^{\Gamma} \hookrightarrow H^{2}\left(\tilde{Y}^{0}, \mathbb{C}\right)=H^{2}\left(\tilde{Y}^{r e g}, \mathbb{C}\right)$. Abusing the notation, by $\lambda_{0} \in$ $H^{2}\left(\tilde{Y}^{0}, \mathbb{C}\right)$ we denote the image of $\lambda_{0} \in \mathfrak{h}_{0}^{*} \subset H^{2}\left(Y^{r e g}, \mathbb{C}\right)$ under the embedding above.

Now let us define a quantization parameter $\lambda^{1}$ for $\tilde{Y}$. Let $\mathcal{L}_{1}^{\prime}, \ldots, \mathcal{L}_{\ell}^{\prime}$ be the codimension 2 leaves of $\tilde{Y}$ and let $\Sigma_{i}^{\prime}=\mathbb{D}^{2} / \Gamma_{i}^{\prime}$ be the corresponding formal slices, $i=1, \ldots, \ell$. We will write $W_{\tilde{Y}, i}, \mathfrak{h}_{\tilde{Y}, i}$ for the factor/summand in $W_{\tilde{Y}}, \mathfrak{h}_{\tilde{Y}}$ corresponding to the index $i$.

Let $\lambda_{i}^{1}, i=1, \ldots, \ell$, denote the quantization parameter for $\Sigma_{i}^{\prime}$ corresponding to $1 \in \mathbb{C} \Gamma_{i}^{\prime}$. Note that this parameter is stable under the action of $N_{\mathrm{SL}_{2}(\mathbb{C})}\left(\Gamma_{i}^{\prime}\right)$ on $\left(\mathbb{C} \Gamma_{i}^{\prime}\right)_{1}^{\Gamma_{i}^{\prime}}$. Hence $\lambda_{i}^{1}$ is stable under the monodromy action of $\pi_{1}\left(\mathcal{L}_{i}^{\prime}\right)$. We set $\lambda^{1}:=\lambda_{0}+\sum_{i=1}^{\ell} \lambda_{i}^{1}$, this is an element of $\mathfrak{h}_{\tilde{Y}}^{*}$.

So we have a filtered quantization $\tilde{\mathcal{A}}_{\lambda^{1}}$ of $\tilde{Y}$.

Lemma 4.2. The action of $\Gamma$ on $\mathbb{C}[\tilde{Y}]$ lifts to a $\Gamma$-action on $\tilde{\mathcal{A}}_{\lambda^{1}}$.

Proof. Since $\Gamma$ acts on $\mathbb{C}[\tilde{Y}]$ by graded Poisson algebra automorphisms, it also acts on the universal quantization $\tilde{\mathcal{A}}_{\mathfrak{h}_{\tilde{Y}}}^{W_{\tilde{Y}}}$ of $\mathbb{C}[\tilde{Y}]$, see [L8, Section 3.7]. We need to show that the parameter $W_{\tilde{Y}} \lambda^{1}$ is $\Gamma$-invariant. The $H^{2}\left(\tilde{Y}^{r e g}, \mathbb{C}\right)$-component of $W_{\tilde{Y}} \lambda^{1}$ is $\Gamma$-stable by the construction. The group $\Gamma$ permutes the codimension 2 symplectic leaves of $\tilde{Y}$ hence induces a permutation of $\{1, \ldots, \ell\}$. The element $\gamma$ gives rise to an isomorphism $\Sigma_{i}^{\prime} \stackrel{\sim}{\rightarrow} \Sigma_{\gamma(i)}^{\prime}$ compatible with the monodromy action. Hence $\gamma$ gives rise to an isomorphism $\mathfrak{h}_{\tilde{Y}, i}^{*} / W_{\tilde{Y}, i} \stackrel{\sim}{\rightarrow} \mathfrak{h}_{\tilde{Y}, \gamma(i)}^{*} / W_{\tilde{Y}, \gamma(i)}$. This isomorphism must come from a diagram automorphism for the Dynkin diagram of $\Gamma_{i}^{\prime}$. Since $\lambda_{i}^{1}$ is invariant under the action of $N_{\mathrm{SL}_{2}(\mathbb{C})}\left(\Gamma_{i}^{\prime}\right)$, we see $\gamma$ takes $W_{\tilde{Y}, i} \lambda_{i}^{1}$ to $W_{\tilde{Y}, \gamma(i)} \lambda_{\gamma(i)}^{1}$. It follows that the parameter $W_{\tilde{Y}} \lambda^{1}$ is $\Gamma$-invariant. 
Let $\tilde{\mathcal{A}}_{\lambda^{1}}^{0}$ denote the microlocalization of $\tilde{\mathcal{A}}_{\lambda^{1}}$ to $\tilde{Y}^{0}$. HC $\tilde{\mathcal{A}}_{\lambda^{1}}^{0}$-bimodules were defined in Section 2.4. By a $\Gamma$-equivariant $\mathrm{HC} \tilde{\mathcal{A}}_{\lambda^{1}}^{0}$-bimodule we mean a $\mathrm{HC} \tilde{\mathcal{A}}_{\lambda^{1}}^{0}$-bimodule $\mathcal{B}$ together with a $\Gamma$-action that is compatible with the actions of $\tilde{\mathcal{A}}_{\lambda^{1}}^{0}$. The category of such $\mathrm{HC}$ bimodules will be denoted by $\mathrm{HC}^{\Gamma}\left(\tilde{\mathcal{A}}_{\lambda^{1}}^{0}\right)$. This is a monoidal category.

Now let us produce a full monoidal embedding $\overline{\mathrm{HC}}\left(\mathcal{A}_{\lambda}\right) \hookrightarrow \mathrm{HC}^{\Gamma}\left(\tilde{\mathcal{A}}_{\lambda^{1}}^{0}\right)$. The construction is based on Proposition 4.1 and follows the construction of an analogous functor in [L3, Section 3.6].

First of all, we have the microlocalization functor $\overline{\mathrm{HC}}\left(\mathcal{A}_{\lambda}\right) \rightarrow \mathrm{HC}\left(\mathcal{A}_{\lambda}^{\text {reg }}\right)$. This functor is a full monoidal embedding.

The quantizations $\mathcal{A}_{\lambda}^{r e g},\left(\tilde{\mathcal{A}}_{\lambda^{1}}^{0}\right)^{\Gamma}$ have the same period, equal to $\lambda_{0}$. So $\left(\tilde{\mathcal{A}}_{\lambda^{1}}^{0}\right)^{\Gamma}$ can be reglued from $\mathcal{A}_{\lambda}^{\text {reg }}$ as explained in Proposition 4.1. Similarly to [L3, Section 3.6], this yields a tensor category equivalence between $\operatorname{HC}\left(\mathcal{A}_{\lambda}^{r e g}\right)$ and $\operatorname{HC}\left(\left(\tilde{\mathcal{A}}_{\lambda^{1}}^{0}\right)^{\Gamma}\right)$ by regluing. On the level of associated graded bimodules, this equivalence is the identity.

Finally, as explained in Section 2.6, the étale morphism $\tilde{Y}^{0} \rightarrow Y^{r e g}$ gives rise to the pullback functor between the categories of Poisson bimodules. Note that the pull-back of a Poisson $R_{\hbar}\left(\mathcal{A}_{\lambda}^{r e g}\right)$-bimodule has a natural $\Gamma$-equivariant structure. Passing to the quotient categories by the $\hbar$-torsion bimodules we get a functor $\operatorname{HC}\left(\left(\tilde{\mathcal{A}}_{\lambda^{1}}^{0}\right)^{\Gamma}\right) \rightarrow \operatorname{HC}^{\Gamma}\left(\tilde{\mathcal{A}}_{\lambda^{1}}^{0}\right)$. This is an equivalence whose inverse is the push-forward functor followed by taking $\Gamma$-invariants.

Summarizing, we get a full monoidal embedding $\operatorname{Loc}_{\tilde{Y}^{0}}: \overline{\mathrm{HC}}\left(\mathcal{A}_{\lambda}\right) \hookrightarrow \mathrm{HC}^{\Gamma}\left(\tilde{\mathcal{A}}_{\lambda^{1}}^{0}\right)$ to be called the localization functor that is the composition

$$
\overline{\mathrm{HC}}\left(\mathcal{A}_{\lambda}\right) \hookrightarrow \operatorname{HC}\left(\mathcal{A}_{\lambda}^{r e g}\right) \stackrel{\sim}{\rightarrow} \operatorname{HC}\left(\left(\tilde{\mathcal{A}}_{\lambda^{1}}^{0}\right)^{\Gamma}\right) \stackrel{\sim}{\rightarrow} \operatorname{HC}^{\Gamma}\left(\tilde{\mathcal{A}}_{\lambda^{1}}^{0}\right) .
$$

4.3. Equivalence $\mathrm{HC}^{\Gamma}\left(\tilde{\mathcal{A}}_{\lambda^{1}}^{0}\right) \cong \mathbb{C} \Gamma$-mod. Our goal now is to describe the monoidal category $\operatorname{HC}^{\Gamma}\left(\tilde{\mathcal{A}}_{\lambda^{1}}^{0}\right)$. First of all, we have a full embedding $\mathbb{C} \Gamma-\bmod \hookrightarrow \operatorname{HC}^{\Gamma}\left(\tilde{\mathcal{A}}_{\lambda^{1}}^{0}\right), V \mapsto$ $V \otimes \tilde{\mathcal{A}}_{\lambda^{1}}^{0}$. We want to prove that it is essentially surjective.

Our first step is the following lemma.

Lemma 4.3. We have $\mathrm{HC}\left(\tilde{\mathcal{A}}_{\lambda^{1}}^{0}\right) \cong \operatorname{Vect}$ (with $\tilde{\mathcal{A}}_{\lambda^{1}}^{0} \in \mathrm{HC}\left(\tilde{\mathcal{A}}_{\lambda^{1}}^{0}\right)$ corresponding to $\mathbb{C} \in$ Vect).

Proof. The proof is in several steps.

Step 1. Let $\mathcal{B} \in \operatorname{HC}\left(\tilde{\mathcal{A}}_{\lambda^{1}}^{0}\right)$. Pick a good filtration on $\mathcal{B}$ and let $\mathcal{B}_{\hbar}$ stand for the $\hbar$-adic completion of $R_{\hbar}(\mathcal{B})$. This is a coherent Poisson $\tilde{\mathcal{A}}_{\lambda^{1} h^{-}}^{0}$-bimodule that is flat over $\mathbb{C}[[\hbar]]$ (here, as before, $h=\hbar^{d}$ ). Let $\iota^{0}: \tilde{Y}^{0} \hookrightarrow \tilde{Y}$ denote the inclusion. We claim that

$$
\iota_{*}^{0}\left(\mathcal{B}_{h}\right) \text { is coherent, } \quad \iota_{*}^{0}\left(\mathcal{B}_{h}\right) / h \iota_{*}^{0}\left(\mathcal{B}_{h}\right) \cong \mathbb{C}[\tilde{Y}]^{\oplus k}
$$

for some $k$.

Step 2. The quotient $\mathcal{B}_{\hbar} / h \mathcal{B}_{\hbar}$ is a graded coherent Poisson $\mathcal{O}_{\tilde{Y}^{0}}$-module. So, by Lemma 2.12, it is isomorphic to $\mathcal{O}_{\tilde{Y}^{0}}^{\oplus k}$. Let $Y_{j}^{\prime}$ denote the spectrum of the completed local ring of a point in the symplectic leaf $\mathcal{L}_{j}^{\prime} \subset \tilde{Y}$. Let us show that

$$
\left.\left.H^{0}\left(Y_{j}^{\prime \times},\left.\mathcal{B}_{\hbar}\right|_{Y_{j}^{\prime \times}}\right)\right|_{Y_{j}^{\prime \times}} \cong \mathcal{B}_{\hbar}\right|_{Y_{j}^{\prime \times}}, \quad H^{0}\left(Y_{j}^{\prime \times},\left.\mathcal{B}_{\hbar}\right|_{Y_{j}^{\prime \times}}\right) / h H^{0}\left(Y_{j}^{\prime \times},\left.\mathcal{B}_{\hbar}\right|_{Y_{j}^{\prime \times}}\right) \cong \mathcal{O}_{Y_{j}^{\prime}}^{\oplus k}
$$

Let us write $\mathbb{D}^{2 n}$ for $\operatorname{Spec}\left(\mathbb{C}\left[\left[x_{1}, y_{1}, \ldots, x_{n}, y_{n}\right]\right]\right)$, the formal symplectic polydisk. Let $\pi_{j}: \mathbb{D}^{2 n} \backslash \mathbb{D}^{2 n-2} \rightarrow Y_{j}^{\prime \times}$ be the quotient morphism for the action of $\Gamma_{j}^{\prime}$. Note that, by 
the construction of $\lambda^{1},\left.\tilde{\mathcal{A}}_{\lambda^{1} h}\right|_{Y_{j}^{\prime \times}}$ is the $\Gamma_{j^{\prime}}^{\prime}$-invariants in the formal Weyl algebra of $\mathbb{D}^{2 n}$ restricted to $\mathbb{D}^{2 n} \backslash \mathbb{D}^{2 n-2}$.

So $\pi_{j}^{*}\left(\left.\mathcal{B}_{\hbar}\right|_{Y_{j}^{\prime \times}}\right)$ is a coherent Poisson bimodule over that restriction. Such a bimodule is nothing else as an $\mathcal{O}[[\hbar]]$-coherent $D_{\mathbb{D}^{2 n} \backslash \mathbb{D}^{2 n-2}}[[\hbar]]$-module (flat over $\mathbb{C}[[\hbar]]$ ). Its (Dmodule) pushforward to $\mathbb{D}^{2 n}$ is an $\mathcal{O}[[\hbar]]$-coherent $D_{\mathbb{D}^{2 n}}[[\hbar]]$-module. So it is $\mathcal{O}_{\mathbb{D}^{2 n}}[[\hbar]] .1$ (4.2) follows.

Step 3. Similarly, choose a smooth point $y$ in $\tilde{Y}^{r e g} \backslash \tilde{Y}^{0}$. The complete analog of (4.2) holds at $y$. Now let $\iota^{\prime}$ denote the inclusion $\tilde{Y}^{0} \hookrightarrow \tilde{Y}^{\text {sreg }}$. By Lemma 3.4, $\iota_{*}^{\prime} \mathcal{B}_{\hbar}$ is a coherent Poisson bimodule. Moreover, $\iota_{*}^{\prime} \mathcal{B}_{\hbar} / h \iota_{*}^{\prime} \mathcal{B}_{\hbar}$ is a vector bundle. It follows that the analog of (4.1) holds for $\iota^{\prime}$ instead of $\iota^{0}$. Then we argue as in the proof of Lemma 3.5 to establish (4.1).

Step 4. In particular, the right $\tilde{\mathcal{A}}_{\lambda^{1} h}$-module $H^{0}\left(\tilde{Y}^{0}, \mathcal{B}_{\hbar}\right)_{\text {fin }}$ is a graded deformation of $\mathbb{C}[\tilde{Y}]^{\oplus k}$ (where different summands can come with different degrees). Such a deformation is unique up to an isomorphism of graded right $\tilde{\mathcal{A}}_{\lambda^{1} h}$-modules. So $H^{0}\left(\tilde{Y}^{0}, \mathcal{B}_{\hbar}\right)$ is a free right $\tilde{\mathcal{A}}_{\lambda^{1} h}$-module. To give a bimodule structure, i.e. a commuting $\tilde{\mathcal{A}}_{\lambda^{1} h^{-}}$action, on $H^{0}\left(\tilde{Y}^{0}, \mathcal{B}_{\hbar}\right)$ amounts to giving an algebra homomorphism $\varphi: \tilde{\mathcal{A}}_{\lambda^{1} h} \rightarrow \operatorname{Mat}_{k}\left(\tilde{\mathcal{A}}_{\lambda^{1} h}\right)$. This homomorphism is the unit $\bmod h$. So it has the form $\mathrm{id}+h \delta+\ldots$, where $\delta \bmod h$ is a matrix $\left(\delta_{i j}\right)_{i, j=1}^{k}$, where $\delta_{i j}$ is a Poisson derivation of $\mathbb{C}[\tilde{Y}]$. By [L8, Proposition 2.14], $\delta_{i j}$ is inner, $\delta_{i j}=\left\{\underline{f}_{i j}, \cdot\right\}$ for $\underline{f}_{i j} \in \mathbb{C}[\tilde{Y}]$. We lift $\underline{f}_{i j}$ to $f_{i j} \in \tilde{\mathcal{A}}_{\lambda^{1} h}$ and form a matrix $F:=\left(f_{i j}\right)$. We have $\varphi=\mathrm{id}+[F, \cdot]+\ldots$, where ... denotes elements starting with $h^{2}$. Then $\varphi \operatorname{Ad}(\exp (-F))-$ id starts in degree at least 2 with respect to $h$. From here and an easy induction (on the smallest degree of $h$ ) we deduce that $\varphi=\operatorname{Ad}(\exp (\tilde{F}))$ for some $\tilde{F} \in \operatorname{Mat}_{k}\left(\tilde{\mathcal{A}}_{\lambda^{1} h}\right)$. So $m \mapsto \exp (\tilde{A}) m$ defines an isomorphism $\iota_{*}^{0}\left(\mathcal{B}_{\hbar}\right) \stackrel{\sim}{\rightarrow} \tilde{\mathcal{A}}_{\lambda^{1} h}^{\oplus k}$ of graded $\tilde{\mathcal{A}}_{\lambda^{1} h^{-}}$-bimodules. This finally implies the claim of the lemma.

Corollary 4.4. The embedding $\mathbb{C} \Gamma-\bmod \hookrightarrow \mathrm{HC}^{\Gamma}\left(\tilde{\mathcal{A}}_{\lambda^{1}}^{0}\right)$ is an equivalence.

Proof. The left inverse is given by $\mathcal{B}$ going to the centralizer of $\tilde{\mathcal{A}}_{\lambda^{1}}$ in $\mathcal{B}$. By Lemma 4.3 this is also the right inverse.

So we get a monoidal full embedding $\bullet_{\dagger}: \overline{\mathrm{HC}}\left(\mathcal{A}_{\lambda}\right) \hookrightarrow \mathbb{C} \Gamma$-mod defined by $\mathcal{B}_{\dagger} \otimes \tilde{\mathcal{A}}_{\lambda^{1}}^{0} \cong$ $\operatorname{Loc}_{\tilde{Y}^{0}}(\mathcal{B})$.

4.4. Properties of $\bullet$. By the construction, one can also recover $\mathcal{B}_{\dagger}$ from a good filtration on $\mathcal{B}$.

Corollary 4.5. Let $\mathcal{B} \in \mathrm{HC}\left(\mathcal{A}_{\lambda}\right)$. Pick a good filtration on $\mathcal{B}$. Then the Poisson $\mathcal{O}_{Y^{r e g}-}$ module gr $\left.\mathcal{B}\right|_{Y^{r e g}}$ is obtained from the $\Gamma$-equivariant Poisson $\mathcal{O}_{\tilde{Y}^{0}}$-module $\mathcal{B}_{\dagger} \otimes \mathcal{O}_{\tilde{Y}^{0}}$ by equivariant descent.

In particular, this corollary shows that $\bullet \nmid$ is independent of the regluing elements $f_{i j}$ from Proposition 4.1.

The next property of $\bullet$ we will need is the existence of a right adjoint functor. This functor will be denoted by $\bullet \dagger$. Namely, let $\psi$ denote the equivalence $\operatorname{HC}\left(\mathcal{A}_{\lambda}^{r e g}\right) \stackrel{\sim}{\rightarrow} \mathbb{C} \Gamma$-mod established in Sections 4.2, 4.3, Then $V^{\dagger}:=H^{0}\left(Y^{r e g}, \psi^{-1}(V)\right)$.

The following properties are proved similarly to the analogous properties established in [L1, Sections 3.4,4] and [L3, Section 3.7].

\footnotetext{
${ }^{1} \mathrm{An}$ argument along these lines has been communicated to me by Shilin Yu.
} 
Lemma 4.6. The functor $\bullet$ † has the following properties:

(1) The kernel and the cokernel of the adjunction unit homomorphism $\mathcal{B} \rightarrow\left(\mathcal{B}_{\dagger}\right)^{\dagger}$ have associated varieties inside $Y^{\text {sing }}$.

(2) The image of $\bullet+$ is closed under taking direct summands.

Property (2) and the claim that $\bullet$ is a monoidal functor imply that $\bullet_{\dagger}$ identifies $\overline{\mathrm{HC}}\left(\mathcal{A}_{\lambda}\right)$ with $\mathbb{C}\left(\Gamma / \Gamma_{\lambda}\right)$-mod for a uniquely determined normal subgroup $\Gamma_{\lambda} \subset \Gamma$. This subgroup is recovered as the intersection of the annihilators of the representations in $\operatorname{im} \bullet$ †

4.5. Consequences. First, we want to give an equivalent formulation of Proposition 3.1 in terms of the functor $\bullet_{\dagger}$. Let us write $\underline{\mathcal{A}}_{\lambda_{i}}^{i}$ for the quantization of $\mathbb{C}^{2} / \Gamma_{i}$ corresponding to the parameter $\lambda_{i}$. Recall that, for each $i$, we have natural homomorphism $\Gamma_{i} \rightarrow \Gamma$, denote it by $\varphi_{i}$.

Proposition 4.7. Let $V \in \operatorname{Rep} \Gamma$. Then the following two claims are equivalent

(i) $V$ lies in the image of $\overline{\mathrm{HC}}\left(\mathcal{A}_{\lambda}\right)$ under $\bullet$ †.

(ii) For all $i=1, \ldots, k$, the pullback $\varphi_{i}^{*}(V)$ lies in the image of the functor $\bullet_{+, \Gamma_{i}}$ : $\overline{\mathrm{HC}}\left(\underline{\mathcal{A}}_{\lambda_{i}}^{i}\right) \hookrightarrow \mathbb{C} \Gamma_{i}$-mod.

Proof. We start with (i) $\Rightarrow\left(\right.$ ii). Let $\mathcal{B} \in \mathrm{HC}\left(\mathcal{A}_{\lambda}\right)$ be such that $\mathcal{B}_{\dagger}=V$. Recall that we have a functor $\bullet_{\dagger, i}: \mathrm{HC}\left(\mathcal{A}_{\lambda}\right) \rightarrow \mathrm{HC}\left(\underline{\mathcal{A}}_{\lambda_{i}}^{i}\right)$. We claim that

$$
\left(\mathcal{B}_{\dagger, i}\right)_{\dagger, \Gamma_{i}}=\varphi_{i}^{*}(V) \text {. }
$$

This will imply (ii).

Let us prove (4.3). Pick a good filtration on $\mathcal{B}$. This induces a good filtration on $\mathcal{B}_{\dagger, i}$. Recall that $\Sigma_{i}$ is the formal slice to $\mathcal{L}_{i}$, so that $\Sigma_{i} \cong \mathbb{D}^{2} / \Gamma_{i}$. The restriction of gr $\mathcal{B}_{\dagger, i}$ to $\left(\mathbb{D}^{2} / \Gamma_{i}\right)^{\times}$coincides with the restriction of gr $\mathcal{B}$ to $\Sigma_{i}^{\times}$by the construction of $\bullet_{\dagger, i}$. On the other hand, by Corollary 4.5, the restriction of gr $\mathcal{B}$ to $Y^{r e g}$ is $\pi_{*}\left(V \otimes \mathcal{O}_{\tilde{Y}_{0}}\right)^{\Gamma}$ and, similarly, the restriction of gr $\mathcal{B}_{\dagger, i}$ to $\left(\mathbb{C}^{2} / \Gamma_{i}\right)^{\times}$is $\pi_{i *}\left(\left(\mathcal{B}_{\dagger, i}\right)_{\dagger, \Gamma_{i}} \otimes \mathcal{O}_{\mathbb{C}^{2 \times}}\right)^{\Gamma_{i}}$. But the restrictions of gr $\left.\mathcal{B}\right|_{Y^{r e g}}$ and gr $\left.\mathcal{B}_{\dagger, i}\right|_{\left(\mathbb{C}^{2} / \Gamma_{i}\right)}$ to $\left(\mathbb{D}^{2} / \Gamma_{i}\right)^{\times}$coincide. The homomorphism $\varphi_{i}: \Gamma_{i} \rightarrow \Gamma$ is the natural homomorphism $\pi_{1}^{a l g}\left(\left(\mathbb{D}^{2} / \Gamma_{i}\right)^{\times}\right) \rightarrow \pi_{1}^{a l g}\left(Y^{r e g}\right)$. (4.3) follows.

Now we prove $(\mathrm{ii}) \Rightarrow(\mathrm{i})$. For this we use Proposition 3.1 . Namely, consider $\mathcal{B}^{\prime} \in$ $\mathrm{HC}\left(\mathcal{A}_{\lambda}^{r e g}\right)$ corresponding to $V$ under the equivalence $\mathrm{HC}\left(\mathcal{A}_{\lambda}^{r e g}\right) \cong \mathbb{C} \Gamma$-mod. This $\mathrm{HC}$ bimodule comes with a natural good filtration, let $\mathcal{B}_{\hbar}^{\prime}$ be the $\hbar$-adic completion of the Rees bimodule of $\mathcal{B}^{\prime}$. Now consider the restriction $\left.\mathcal{B}_{\hbar}^{\prime}\right|_{Y_{i}^{\times}}$.

We claim that for all $i$ we have

$\left(^{*}\right)$ the restriction of $H^{0}\left(Y_{i}^{\times},\left.\mathcal{B}_{\hbar}^{\prime}\right|_{Y_{i}^{\times}}\right)$to $Y_{i}^{\times}$is $\left.\mathcal{B}_{\hbar}^{\prime}\right|_{Y_{i}^{\times}}$.

Once this is known, the proof of $V \in \operatorname{im}\left(\bullet_{\dagger}\right)$ follows from Proposition 3.1.

In the proof of $\left(^{*}\right)$ our first goal is to extend $\left.\mathcal{B}_{\hbar}^{\prime}\right|_{Y_{i}^{\times}}$to $\left(\mathbb{C}^{2 n} \backslash \mathbb{C}^{2 n-2}\right) / \Gamma_{i}$. Thanks to Lemma 2.14, we can equip $\mathcal{B}_{\hbar}^{\prime}$ with an Euler derivation. Consider the $\left.R_{\hbar}^{\wedge}\left(\mathcal{A}_{\lambda}\right)\right|_{Y_{i}}{ }^{-}$ module $H^{0}\left(Y_{i}^{\times},\left.\mathcal{B}_{\hbar}^{\prime}\right|_{Y_{i}^{\times}} /\left.\hbar^{m} \mathcal{B}_{\hbar}^{\prime}\right|_{Y_{i}^{\times}}\right)$. It inherits the Euler derivation from $\left.\mathcal{B}_{\hbar}^{\prime}\right|_{Y_{i}^{\times}}$, denote it also by eu. Choose a $\mathbb{Z}$-equivariant map $\alpha: \mathbb{C} \rightarrow \mathbb{Z}$. Define a $\mathbb{C}^{\times}$-action on the eu-finite part of $H^{0}\left(Y_{i}^{\times},\left.\mathcal{B}_{\hbar}^{\prime}\right|_{Y_{i}^{\times}} /\left.\hbar^{m} \mathcal{B}_{\hbar}^{\prime}\right|_{Y_{i}^{\times}}\right)$by declaring that $t \in \mathbb{C}^{\times}$acts by $t^{\alpha(z)}$ on the generalized eigenspace for eu with eigenvalue $z$. This extends to a pro-rational $\mathbb{C}^{\times}$-action on $H^{0}\left(Y_{i}^{\times},\left.\mathcal{B}_{\hbar}^{\prime}\right|_{Y_{i}^{\times}} /\left.\hbar^{m} \mathcal{B}_{\hbar}^{\prime}\right|_{Y_{i}^{\times}}\right)$. Consider the $\mathbb{C}^{\times}$-finite part $H^{0}\left(Y_{i}^{\times},\left.\mathcal{B}_{\hbar}^{\prime}\right|_{Y_{i}^{\times}} /\left.\hbar^{m} \mathcal{B}_{\hbar}^{\prime}\right|_{Y_{i}^{\times}}\right)_{f i n}$. This is a module over $R_{\hbar}\left(\mathbb{A} \otimes \underline{\mathcal{A}}_{\lambda_{i}}^{i}\right) /\left(\hbar^{m}\right)$. The pullback to $Y_{i}^{\times}$of

$$
H^{0}\left(Y_{i}^{\times},\left.\mathcal{B}_{\hbar}^{\prime}\right|_{Y_{i}^{\times}} /\left.\hbar^{m} \mathcal{B}_{\hbar}^{\prime}\right|_{Y_{i}^{\times}}\right) / \hbar^{m-1} H^{0}\left(Y_{i}^{\times},\left.\mathcal{B}_{\hbar}^{\prime}\right|_{Y_{i}^{\times}} /\left.\hbar^{m} \mathcal{B}_{\hbar}^{\prime}\right|_{Y_{i}^{\times}}\right) \rightarrow H^{0}\left(Y_{i}^{\times},\left.\mathcal{B}_{\hbar}^{\prime}\right|_{Y_{i}^{\times}} /\left.\hbar^{m-1} \mathcal{B}_{\hbar}^{\prime}\right|_{Y_{i}^{\times}}\right)
$$


is an isomorphism. It follows that the kernel and the cokernel of this homomorphism are supported on $Y_{i} \cap \mathcal{L}_{i}$. Therefore after passing to $\mathbb{C}^{\times}$-finite part we have that the kernel and the cokernel are still supported on the closed leaf. Let $\left(\left.\mathcal{B}_{\hbar}^{\prime}\right|_{Y_{i}^{\times}} /\left.\hbar^{m} \mathcal{B}_{\hbar}^{\prime}\right|_{Y_{i}^{\times}}\right)_{\text {fin }}$ be the microlocalization of $H^{0}\left(Y_{i}^{\times},\left.\mathcal{B}_{\hbar}^{\prime}\right|_{Y_{i}^{\times}} /\left.\hbar^{m} \mathcal{B}_{\hbar}^{\prime}\right|_{Y_{i}^{\times}}\right)_{\text {fin }}$ to $\left(\mathbb{C}^{2 n} \backslash \mathbb{C}^{2 n-2}\right) / \Gamma_{i}$. We set $\mathcal{B}_{\hbar, i, e x t}^{\prime}:=$ $\lim _{\longleftarrow}\left(\left.\mathcal{B}_{\hbar}^{\prime}\right|_{Y_{i}^{\times}} /\left.\hbar^{m} \mathcal{B}_{\hbar}^{\prime}\right|_{Y_{i}^{\times}}\right)_{f i n}$. This is a graded coherent Poisson module over the microlocalization of the $\hbar$-adic completion of $R_{\hbar}\left(\mathbb{A} \otimes \underline{\mathcal{A}}_{\lambda_{i}}^{i}\right)$. Its pullback to $Y_{i}^{\times}$is $\left.\mathcal{B}_{\hbar}^{\prime}\right|_{Y_{i}^{\times}}$. So $\mathcal{B}_{\hbar, i, e x t}^{\prime}$ is an extension we want.

Let $\mathcal{B}_{\hbar, i, f i n}^{\prime}$ denote the $\mathbb{C}^{\times}$-finite part of $\mathcal{B}_{\hbar, i, \text { ext }}^{\prime}$. Set $\mathcal{B}_{i}^{\prime}:=\mathcal{B}_{\hbar, i, f i n}^{\prime} /(\hbar-1) \mathcal{B}_{\hbar, i, f i n}^{\prime}$. This is an object of

$$
\mathrm{HC}\left(\left[\mathbb{A} \otimes \underline{\mathcal{A}}_{\lambda_{i}}^{i}\right]_{\left(\mathbb{C}^{2 n} \backslash \mathbb{C}^{2 n-2}\right) / \Gamma_{i}}\right) .
$$

By the construction, its image under the equivalence of the latter category with $\operatorname{Rep}\left(\Gamma_{i}\right)$ is $\varphi_{i}^{*}(V)$. It follows that $\mathcal{B}_{i}^{\prime}$ extends to an object of $\mathrm{HC}\left(\mathbb{A} \otimes \underline{\mathcal{A}}_{\lambda_{i}}^{i}\right)$. This implies $\left.{ }^{*}\right)$ and hence finishes the proof.

Now we proceed to the second part of this section, where we compare the categories $\overline{\mathrm{HC}}(\hat{\mathcal{A}})$ and $\overline{\mathrm{HC}}(\mathcal{A})$, where $\hat{\mathcal{A}}$ is a filtered quantization of $\hat{Y}$ and $\mathcal{A}:=\hat{\mathcal{A}}^{\Gamma / \Gamma^{\prime}}$. Here $\hat{Y}:=\operatorname{Spec}\left(\mathbb{C}\left[\hat{Y}^{0}\right]\right)$ for a finite etale cover $\hat{Y}^{0}$ of $Y^{\text {reg }}$ with Galois group $\Gamma / \Gamma^{\prime}$.

Proposition 4.8. Let $V$ be a $\mathbb{C} \Gamma$-module and let $V^{\prime}$ be its restriction to $\Gamma^{\prime}$. In the notation above, the following two conditions are equivalent.

(1) $V$ lies in the image of $\overline{\mathrm{HC}}(\mathcal{A})$ under $\bullet$.

(2) $V^{\prime}$ lies in the image of $\overline{\mathrm{HC}}(\hat{\mathcal{A}})$ under $\bullet_{\dagger^{\prime}}$ (this is our notation for the $\bullet \nmid$ for $\left.\hat{Y}\right)$.

Proof. Let $\bullet \dagger^{\prime \prime}$ denote the right adjoint for $\bullet_{\dagger^{\prime}}$. We claim that there is a natural $\Gamma / \Gamma^{\prime}$-action on $V^{\dagger^{\prime}}$ such that

$$
V^{\dagger} \cong\left(V^{\dagger^{\prime}}\right)^{\Gamma / \Gamma^{\prime}}
$$

We choose an affine covering $U_{i}$ of $Y^{r e g}$. Let $\pi^{\prime}: \hat{Y}^{0} \rightarrow Y^{r e g}$ be the quotient morphism for the $\Gamma / \Gamma^{\prime}$-action on $\hat{Y}^{0}$. Set $U_{i}^{\prime}:=\pi^{\prime-1}\left(U_{i}\right)$.

Consider the quantization $\tilde{\mathcal{A}}:=\tilde{\mathcal{A}}_{\lambda^{1}}$, where $\lambda^{1}$ is constructed from $\lambda$, a quantization parameter for $\mathcal{A}$. Let $f_{i j}$ be the elements used to reglue $\left.\mathcal{A}\right|_{Y^{\text {reg }}}$ to $\left.\tilde{\mathcal{A}}^{\Gamma}\right|_{Y^{\text {reg }}}$. Their pullbacks $\pi^{* *}\left(f_{i j}\right)$ are then used to reglue $\left.\hat{\mathcal{A}}\right|_{\hat{Y}^{0}}$ to $\left.\tilde{\mathcal{A}}^{\Gamma^{\prime}}\right|_{\hat{Y}^{0}}$. This gives rise to an equivalence $\psi^{\prime}: \operatorname{HC}^{\Gamma / \Gamma^{\prime}}\left(\left.\hat{\mathcal{A}}\right|_{\hat{Y}^{0}}\right) \stackrel{\sim}{\rightarrow} \mathbb{C} \Gamma$-mod. The right adjoint of the resulting functor $\operatorname{HC}^{\Gamma / \Gamma^{\prime}}(\hat{\mathcal{A}}) \rightarrow$ $\mathbb{C} \Gamma$-mod is given by $V \mapsto H^{0}\left(\hat{Y}^{0}, \psi^{\prime-1}(V)\right)$. The object $V^{\dagger^{\prime}} \in \operatorname{HC}(\hat{\mathcal{A}})$ is obtained from $H^{0}\left(\hat{Y}^{0}, \psi^{\prime-1}(V)\right) \in \mathrm{HC}^{\Gamma / \Gamma^{\prime}}(\hat{\mathcal{A}})$ by forgetting the action of $\Gamma / \Gamma^{\prime}$. So $\Gamma / \Gamma^{\prime}$ acts on $V^{\dagger^{\prime}}$ and $V^{\dagger}=\left(V^{\dagger^{\prime}}\right)^{\Gamma / \Gamma^{\prime}}$.

Also note that for $\mathcal{B} \in \mathrm{HC}^{\Gamma / \Gamma^{\prime}}(\hat{\mathcal{A}})$ we have a natural identification

$$
\mathcal{B}_{\dagger^{\prime}}=\left(\mathcal{B}^{\Gamma / \Gamma^{\prime}}\right)_{\dagger}
$$

Now we are ready to prove that conditions (1) and (2) in the statement of the proposition are equivalent. Recall that, by Lemma 4.6, $\bullet^{\dagger}: \mathbb{C} \Gamma-\bmod \rightarrow \overline{\mathrm{HC}}\left(\mathcal{A}_{\lambda}\right)$ is left inverse to $\bullet_{\dagger}$. So $V$ lies in the image of $\bullet \dagger$ if and only if $V=\left(V^{\dagger}\right)_{\dagger}$. Similarly, $V$ lies in the image of $\bullet \dagger^{\prime}$ if and only if $V=\left(V^{\dagger^{\prime}}\right)_{\dagger^{\prime}}$. Using (4.4) and (4.5), we see that $\left(V^{\dagger^{\prime}}\right)_{\dagger^{\prime}} \cong\left(V^{\dagger}\right)_{\dagger^{\prime}}$. The equivalence $(1) \Leftrightarrow(2)$ follows. 
4.6. Translation equivalences. Let $\lambda \in \mathfrak{h}_{Y}^{*}$. It turns out that for certain values of $\lambda^{\prime}$ the image of $\overline{\mathrm{HC}}\left(\mathcal{A}_{\lambda^{\prime}}\right)$ in $\mathbb{C} \Gamma$-mod coincides with the image of $\overline{\mathrm{HC}}\left(\mathcal{A}_{\lambda}\right)$.

Namely, let us define the "weight lattice" $\Lambda_{Y} \subset \mathfrak{h}_{Y}^{*}$. By definition, it is the image of $\operatorname{Pic}\left(X^{r e g}\right)$ in $\mathfrak{h}_{Y}^{*}$, where $X$ is a $\mathbb{Q}$-terminalization of $Y$.

Then we can form the "extended affine Weyl group" $W_{Y}^{a e}:=W_{Y} \ltimes \Lambda_{Y}$.

Lemma 4.9. Let $\lambda^{\prime} \in W_{Y}^{a e} \lambda$. Then there is an equivalence $\overline{\mathrm{HC}}\left(\mathcal{A}_{\lambda}\right) \stackrel{\sim}{\rightarrow} \overline{\mathrm{HC}}\left(\mathcal{A}_{\lambda^{\prime}}\right)$ intertwining the inclusions $\overline{\mathrm{HC}}\left(\mathcal{A}_{\lambda}\right), \overline{\mathrm{HC}}\left(\mathcal{A}_{\lambda^{\prime}}\right) \hookrightarrow \mathbb{C} \Gamma$-mod.

Proof. For $\lambda^{\prime} \in W_{Y} \lambda$, the algebras $\mathcal{A}_{\lambda}, \mathcal{A}_{\lambda^{\prime}}$ are the same and the claim of the lemma follows. So it remains to consider the situation when $\lambda^{\prime}-\lambda \in \Lambda_{Y}$.

We can speak about HC $\mathcal{A}_{\lambda^{\prime}} \mathcal{A}_{\lambda^{-}}$-bimodules. Here is an example. Lift $\lambda^{\prime}-\lambda$ to an element $\chi \in \operatorname{Pic}\left(X^{r e g}\right)$. Consider the line bundle $\mathcal{O}(\chi)$ on $X^{r e g}$. Since $H^{i}\left(X^{r e g}, \mathcal{O}\right)=0$ for $i=1,2$, we see that $\mathcal{O}(\chi)$ admits a unique filtered deformation to a $\mathcal{D}_{\lambda^{\prime}}^{r e g}-\mathcal{D}_{\lambda}^{r e g}$-bimodule, compare with [BPW, Section 5.1]. The deformed bimodules will be denoted by $\mathcal{D}_{\lambda, \chi}^{r e g}$. Set $\mathcal{A}_{\lambda, \chi}:=\Gamma\left(\mathcal{D}_{\lambda, \chi}\right)$. This is a $\mathrm{HC} \mathcal{A}_{\lambda^{\prime}} \mathcal{A}_{\lambda^{-}}$-bimodule. Similarly, we can consider the HC $\mathcal{A}_{\lambda^{-}} \mathcal{A}_{\lambda^{\prime}}$-bimodule $\mathcal{A}_{\lambda^{\prime},-\chi}$. The restrictions $\mathcal{A}_{\lambda, \chi}^{r e g}, \quad \mathcal{A}_{\lambda^{\prime},-\chi}^{\text {reg }}$ to $Y^{r e g}$ are mutually inverse. It follows that the functors

$$
\mathcal{A}_{\lambda, \chi} \otimes_{\mathcal{A}_{\lambda}} \bullet \otimes_{\mathcal{A}_{\lambda}} \mathcal{A}_{\lambda^{\prime},-\chi}, \mathcal{A}_{\lambda^{\prime},-\chi} \otimes_{\mathcal{A}_{\lambda^{\prime}}} \bullet \otimes_{\mathcal{A}_{\lambda^{\prime}}} \mathcal{A}_{\lambda, \chi}
$$

are mutually inverse equivalences $\overline{\mathrm{HC}}\left(\mathcal{A}_{\lambda}\right) \leftrightarrows \overline{\mathrm{HC}}\left(\mathcal{A}_{\lambda^{\prime}}\right)$

It remains to show that these equivalences intertwine the embeddings $\overline{\mathrm{HC}}\left(\mathcal{A}_{\lambda}\right), \overline{\mathrm{HC}}\left(\mathcal{A}_{\lambda^{\prime}}\right) \hookrightarrow$ $\mathbb{C} \Gamma$-mod. To check this, for $\mathcal{B} \in \operatorname{HC}\left(\mathcal{A}_{\lambda}\right)$, we need to establish a good filtration on

$$
\mathcal{A}_{\lambda, \chi} \otimes_{\mathcal{A}_{\lambda}} \mathcal{B} \otimes_{\mathcal{A}_{\lambda}} \mathcal{A}_{\lambda^{\prime},-\chi}
$$

in a natural way such that the restriction of its associated graded to $Y^{\text {reg }}$ is naturally identified with gr $\left.\mathcal{B}\right|_{Y^{r e g}}$. For this we take the natural filtration of the tensor product bimodule on (4.6). Since gr $\left.\left.\mathcal{A}_{\lambda, \chi}\right|_{Y^{\text {reg }}} \cong \mathcal{O}(\chi)\right|_{Y^{\text {reg }}}$ and gr $\left.\left.\mathcal{A}_{\lambda^{\prime},-\chi}\right|_{Y^{\text {reg }}} \cong \mathcal{O}(-\chi)\right|_{Y^{\text {reg }}}$ are invertible we see that

$$
\left.\left.\left.\left.\left.\operatorname{gr}\left(\mathcal{A}_{\lambda, \chi} \otimes_{\mathcal{A}_{\lambda}} \mathcal{B} \otimes_{\mathcal{A}_{\lambda}} \mathcal{A}_{\lambda^{\prime},-\chi}\right)\right|_{Y^{r e g}} \cong \mathcal{O}(\chi)\right|_{Y^{r e g}} \otimes \operatorname{gr} \mathcal{B}\right|_{Y^{r e g}} \otimes \mathcal{O}(-\chi)\right|_{Y^{r e g}} \cong \operatorname{gr} \mathcal{B}\right|_{Y^{r e g}}
$$

This finishes the proof of the lemma.

\section{Classification for quantizations of Kleinian singularities}

The goal of this section is to prove a more precise version of Theorem [1.2, Recall that $\mathfrak{g}, W_{\Gamma}, \mathfrak{h}_{\Gamma}$ denote the Lie algebra, Weyl group and the Cartan space of the same type as $\Gamma, \Lambda_{r} \subset \mathfrak{h}_{\Gamma}^{*}$ is the root lattice, and $W_{\Gamma}^{a}:=W_{\Gamma} \ltimes \Lambda_{r}$ is the affine Weyl group.

Theorem 5.1. Let $\Gamma \subset \mathrm{SL}_{2}(\mathbb{C})$ be a finite subgroup not of type $E_{8}$. The following claims are true:

(1) For each $c \in(\mathbb{C} \Gamma)_{1}^{\Gamma}$, there is a minimal normal subgroup $\Gamma_{c} \subset \Gamma$ such that $W_{\Gamma}^{a} \lambda_{c}$ contains $\lambda_{c^{\prime}}$ with $c^{\prime} \in \mathbb{C} \Gamma_{c}(\subset \mathbb{C} \Gamma)$.

(2) Let $\lambda \in \mathfrak{h}_{\Gamma}^{*}$ be the parameter corresponding to $c$. Then the image of $\overline{\mathrm{HC}}\left(\mathcal{A}_{\lambda}\right)$ in $\mathbb{C} \Gamma$-mod under $\bullet$ is $\mathbb{C}\left(\Gamma / \Gamma_{c}\right)$-mod.

The scheme of the proof of (2) is, essentially, as follows. First, we show that a onedimensional representation of $\Gamma$ lies in the image of $\bullet$ if and only if $\Gamma_{c}$ acts trivially on it. For this we use the known description of $\overline{\mathrm{HC}}\left(\mathcal{U}_{\lambda}\right)$ (here $\mathcal{U}_{\lambda}$ is the central reduction of the universal enveloping algebra $\mathcal{U}:=U(\mathfrak{g}))$ and Proposition 4.7 that allows us to relate 
$\overline{\mathrm{HC}}\left(\mathcal{U}_{\lambda}\right)$ and $\overline{\mathrm{HC}}\left(\mathcal{A}_{\lambda}\right)$. To extend (2) to higher dimensional irreducible representations of $\Gamma$ we use translation equivalences and Proposition 4.8.

5.1. One-dimensional representations in the image of $\bullet$. Our first task is to describe the one-dimensional representations of $\Gamma$ lying in the image of $\bullet_{\uparrow}$. The initial step is to recall the description of the category $\overline{\mathrm{HC}}\left(\mathcal{U}_{\lambda}\right)$.

Below we write $\mathfrak{h}, W, W^{a}$ for $\mathfrak{h}_{\Gamma}, W_{\Gamma}, W_{\Gamma}^{a}$, we view $\mathfrak{h}$ as a Cartan subalgebra of $\mathfrak{g}$. Let $\Lambda$ denote the weight lattice in $\mathfrak{h}^{*}$. Note that $\left(\Lambda / \Lambda_{r}\right)^{*}$ coincides with $\Gamma_{\mathfrak{g}}:=\pi_{1}\left(Y^{\text {reg }}\right)$, where $Y$ stands for the nilpotent cone. Form the extended affine Weyl group $W^{a e}:=W \ltimes \Lambda$ so that $W^{a}$ is a normal subgroup in $W^{a e}$ and $W^{a e} / W^{a} \cong \Lambda / \Lambda_{r}$.

Here is a description of $\overline{\mathrm{HC}}\left(\mathcal{U}_{\lambda}\right)$. It is standard but since we haven't found a proof in the literature, we provide it.

Lemma 5.2. The image under $\bullet$ of $\overline{\operatorname{HC}}\left(\mathcal{U}_{\lambda}\right)$ in $\operatorname{Rep}\left[\left(\Lambda / \Lambda_{r}\right)^{*}\right]$ coincides with the subcategory of representations whose irreducible constituents lie in $W_{\lambda}^{a e} / W_{\lambda}^{a}$ (naturally embedded into $\left.W^{a e} / W^{a}\right)$.

Proof. In the proof we can assume that $\lambda$ is regular. Indeed, if $\lambda^{\prime}-\lambda \in \Lambda$, then the images of $\overline{\mathrm{HC}}\left(\mathcal{U}_{\lambda}\right)$ and $\overline{\mathrm{HC}}\left(\mathcal{U}_{\lambda^{\prime}}\right)$ in $\mathbb{C}\left(\Lambda / \Lambda_{r}\right)^{*}$-mod are the same, as was explained in Lemma 4.9 (in the case of general $Y$ ).

Note that to an irreducible $\mathrm{HC} \mathcal{U}$-bimodule, $\mathcal{B}$, we can assign an element of $\Lambda / \Lambda_{r}$ as follows: this is the $\Lambda_{r}$-coset of weights of $\mathfrak{g}$ in its adjoint (and hence locally finite) action on $\mathcal{B}$. Since $\mathcal{B}$ is irreducible, we have $\mathcal{B}=\mathcal{U} \mathfrak{U} \mathcal{U}$ for any $b \in \mathcal{B}$. Hence all the weights lie in a single coset.

Let us show that the irreducible $\Gamma_{\mathfrak{g}}$-module corresponding to this element coincides with $\mathcal{B}_{\dagger}$. Indeed, $\Gamma_{\mathfrak{g}}$ is identified with the center $Z$ of the simply connected algebraic group $G$ with Lie algebra $\mathfrak{g}$. In our case - when $Y$ is the nilpotent cone - the functor $\bullet$ constructed in the end of Section 4.3 is a special case of the functor $\bullet$ constructed in [L1, Section 3.4] in the case of the principal orbit. The source of that functor is the category $\mathrm{HC}(\mathcal{U})$ of all $\mathrm{HC} \mathcal{U}$-bimodules and the target is the category $\operatorname{HC}^{Z}(\mathcal{W})$ of $Z$-equivariant $\mathrm{HC}$ bimodules over the $\mathrm{W}$-algebra $\mathcal{W}$ constructed for the principal orbit. This $\mathrm{W}$-algebra coincides with the center of $\mathcal{U}$. By the construction of the functor in [L1] it is clear that if $Z$ acts on $\mathcal{B}$ via a character $\chi$, then it acts on $\mathcal{B}_{\dagger}$ via that character as well. This shows the claim on the coincidence of two $\Gamma_{\mathfrak{g}}$-modules in the beginning of the paragraph.

So we need to understand the set $\left\{\lambda+\Lambda_{r}\right\}$, where $\lambda$ runs over the possible weights of $\mathrm{HC}$ bimodules in $\overline{\mathrm{HC}}\left(\mathcal{U}_{\lambda}\right)$. All classes from $W_{\lambda}^{a e} / W_{\lambda}^{a}$ are realized by translation bimodules that, for general $Y$, were introduced in the proof of Lemma 4.9, So we need to show that no other classes appear. All weights that appear in $\mathcal{B} \in \mathrm{HC}\left(\mathcal{U}_{\lambda}\right)$ also appear in $\operatorname{pr}_{\lambda}\left(V \otimes \mathcal{U}_{\lambda}\right)$, where $V$ is a finite dimensional $\mathfrak{g}$-module and $\operatorname{pr}_{\lambda}$ stands for the projection to the infinitesimal block with central character $\lambda$. Also note that for every $\mathcal{B} \in \operatorname{HC}\left(\mathcal{U}_{\lambda}\right)$ there is a Verma module $\Delta(w \cdot \lambda)$ for $w \in W$ in the infinitesimal block $\mathcal{O}_{\lambda}$ of the BGG category $\mathcal{O}$ such that $\mathcal{B} \otimes \mathcal{U}_{\lambda} \Delta(w \cdot \lambda) \neq 0$, this follows, for example, from [BG]. So we reduce to showing that all weights that appear in $\operatorname{pr}_{\lambda}(V \otimes \Delta(w \cdot \lambda))$ are of the form $w \cdot \lambda+\chi$ with $\chi+\Lambda_{r} \in W_{\lambda}^{a e} / W_{\lambda}^{a}$. On the other hand, any weight appearing in $\operatorname{pr}_{\lambda}(V \otimes \Delta(w \cdot \lambda))$ should appear in $\Delta(u \cdot \lambda)$ for some $u \in W$ hence lies in $W^{a} \lambda$. Clearly, $w \cdot \lambda+\chi \in W^{a} \lambda$ is equivalent to $\chi+\Lambda_{r} \in W_{\lambda}^{a e} / W_{\lambda}^{a}$, which is precisely what we need. 
With Lemma 5.2 we can now use Proposition 4.7 to describe the one-dimensional representations of $\Gamma$ in the image of $\operatorname{HC}\left(\mathcal{A}_{\lambda}\right)$. For this, note that $\Gamma /(\Gamma, \Gamma) \cong \Gamma_{\mathfrak{g}}$. So the one-dimensional $\Gamma$-modules are in a one-to-one correspondence with $\Lambda / \Lambda_{r}$.

Proposition 5.3. Let $V$ be a one-dimensional $\Gamma$-module. Then the following claims are equivalent:

(1) $V$ lies in $W_{\lambda}^{a e} / W_{\lambda}^{a}$.

(2) $V$ lies in the image of $\mathrm{HC}\left(\mathcal{A}_{\lambda}\right)$ under $\bullet$ +

Proof. The variety $Y$ has a unique symplectic leaf of codimension 2 and the corresponding Kleinian group $\Gamma$ has the same type as $\mathfrak{g}$. Note the homomorphism $\Gamma \rightarrow \Gamma_{\mathfrak{g}}$ of algebraic fundamental groups is an epimorphism (hence gives an isomorphism $\Gamma /(\Gamma, \Gamma) \stackrel{\sim}{\rightarrow} \Gamma_{\mathfrak{g}}$ ). Indeed, assume that $\Gamma \rightarrow \Gamma_{\mathfrak{g}}$ is not surjective. Equivalently, there is a nontrivial irreducible $\Gamma_{\mathfrak{g}}$-module, say $V$, with trivial pull-back to $\Gamma$. By Proposition 4.7, $V$ lies in the image of $\mathrm{HC}\left(\mathcal{U}_{\lambda}\right)$ for all $\lambda$. This contradicts Lemma 5.2. The surjectivity can also be checked case by case.

According to Lemma 5.2, (1) is equivalent to $V$ lying in the image of $\overline{\mathrm{HC}}\left(\mathcal{U}_{\lambda}\right)$. The latter condition is equivalent to (2), this is a special case of Proposition 4.7.

5.2. Subgroup $\Gamma_{c}$. In this section, to a parameter $\lambda \in \mathfrak{h}_{\Gamma}^{*}$ or, equivalently, $c \in(\mathbb{C} \Gamma)_{1}^{\Gamma}$ we assign a normal subgroup $\Gamma_{c}$. The construction will be inductive. Let $\Gamma_{c}^{\prime}$ be the normal subgroup in $\Gamma$ that is the intersection of the kernels of the one-dimensional representations of $\Gamma$ that lie in $W_{\lambda}^{a e} / W_{\lambda}^{a}$. So $\left(\Gamma / \Gamma_{c}^{\prime}\right)^{*}=W_{\lambda}^{a e} / W_{\lambda}^{a}$.

Lemma 5.4. There is an element $\lambda^{\prime} \in W^{a} \lambda$ such that the corresponding parameter $c^{\prime}$ lies in $\mathbb{C} \Gamma_{c}^{\prime}$.

Proof. In the proof of the lemma we can assume that $\lambda$ is real, i.e., $\lambda \in \mathbb{R} \otimes_{\mathbb{Z}} \Lambda$. Indeed, the locus of $\lambda$ such that $c^{\prime} \in \mathbb{C} \Gamma_{c}^{\prime}$ is the union of affine subspaces of $\mathfrak{h}^{*}$ defined over $\mathbb{R}$. Similarly, the locus of parameters $\lambda$ with given group $W_{\lambda}^{a e} / W_{\lambda}^{a}$ is the union of affine subspaces of $\mathfrak{h}^{*}$ defined over $\mathbb{R}$.

We claim that for $\lambda^{\prime} \in W^{a} \lambda$ lying in the fundamental alcove we have $c^{\prime} \in \mathbb{C} \Gamma_{c}^{\prime}$. We have $W^{a e}=\left(\Lambda / \Lambda_{r}\right) \ltimes W^{a}$ and $W_{\lambda^{\prime}}^{a e} / W_{\lambda^{\prime}}^{a}=\left(\Lambda / \Lambda_{r}\right)_{\lambda^{\prime}}$, where we view $\Lambda / \Lambda_{r}$ as a group acting on the fundamental alcove. The action of $\Lambda / \Lambda_{r}$ comes from automorphisms of the affine Dynkin diagram. Let $\mathfrak{A}_{\lambda^{\prime}}$ be the group of the automorphisms of the affine Dynkin diagram coming from $\left(\Lambda / \Lambda_{r}\right)_{\lambda^{\prime}}$. The group $\Gamma_{c}^{\prime}$ is the largest normal subgroup $\Gamma_{1} \subset \Gamma$ such that the $\Gamma$-irreducibles in the same $\mathfrak{A}_{\lambda^{\prime}}$-orbit become isomorphic over $\Gamma_{1}$. This follows from the observation that the action of $\left(\Lambda / \Lambda_{r}\right)_{\lambda^{\prime}}$ on the $\Gamma$-irreducibles is by tensoring with the one-dimensional $\Gamma / \Gamma_{c}^{\prime}$-modules.

Now a case by case analysis shows that $c^{\prime} \in \mathbb{C} \Gamma_{c}^{\prime}$ if $\lambda^{\prime}$ lies in the fundamental alcove and $\left(\Lambda / \Lambda_{r}\right)_{\lambda^{\prime}}^{*}=\Gamma / \Gamma_{c}^{\prime}$.

We define a sequence of normal in $\Gamma$ subgroups $\Gamma_{c}^{\prime} \supset \Gamma_{c}^{\prime \prime} \supset \ldots$ as follows. We set $\Gamma_{c}^{\prime \prime}:=\left(\Gamma_{c^{\prime}}^{\prime}\right)^{\prime}$. Let $\mathfrak{h}_{1}$ and $W_{1}$ be the Cartan space and the finite Weyl group for $\Gamma_{c}^{\prime}$. Inside $\mathfrak{h}_{1}$ we have the subspace of $\Gamma / \Gamma_{c}^{\prime}$-invariant elements, denote it by $\underline{\mathfrak{h}}_{1}$ (the action of $\Gamma / \Gamma_{c}^{\prime}$ comes from twisting the irreducible $\Gamma_{c^{\prime}}$-modules so it is by diagram automorphisms). Note that $\underline{\mathfrak{h}}_{1}^{*}$ naturally embeds into $\mathfrak{h}^{*}$ and under the affine identification $\mathfrak{h}^{*} \cong(\mathbb{C} \Gamma)_{1}^{\Gamma}$, the space $\underline{\mathfrak{h}}_{1}^{*}$ is identified with $\left(\mathbb{C} \Gamma_{c}^{\prime}\right)_{1}^{\Gamma}$.

Set $\underline{W}_{1}:=W_{1}^{\Gamma / \Gamma_{c}^{\prime}}$, this group acts faithfully on $\underline{\mathfrak{h}}_{1}$. The simple roots for $\left(\underline{\mathfrak{h}}_{1}, \underline{W}_{1}\right)$ are exactly the elements of the form $\sum_{\alpha \in \mathfrak{O}} \alpha$, where $\mathfrak{O}$ runs over the set of orbits of $\Gamma / \Gamma_{c}^{\prime}$ on 
the set of simple roots for $W_{1}$. The simple coroots have the same description. It follows that the root lattice $\underline{\Lambda}_{1}^{\prime}$ for $\underline{W}_{1}$ coincides with the intersection $\Lambda_{1}^{\prime} \cap \underline{\mathfrak{h}}_{1}^{*}$ (and the similar claim holds for the coroot lattice). Also the fundamental chamber for $\left(\underline{W}_{1}, \underline{\mathfrak{h}}_{1}\right)$ is the intersection of that for $\left(W_{1}, \mathfrak{h}_{1}\right)$ with $\underline{\mathfrak{h}}_{1}$. The maximal roots and coroots for $\underline{\mathfrak{h}}_{1}$ and $\underline{\mathfrak{h}}_{1}$ coincide. It follows that the fundamental alcove in $\underline{\mathfrak{h}}_{1 \mathbb{R}}^{*}$ coincides with the intersection of $\underline{\mathfrak{h}}_{1 \mathbb{R}}^{*}$ and the fundamental alcove in $\mathfrak{h}_{1 \mathbb{R}}^{*}$. In particular, we have the following analog of Lemma 5.4 (note that if we replace $\underline{W}_{1}^{a}$ with $W_{1}^{a}$ this is just Lemma 5.4).

Lemma 5.5. There is an element $\lambda^{\prime \prime} \in \underline{W}_{1}^{a} \lambda^{\prime}$ such that the corresponding parameter $c^{\prime \prime}$ lies in $\mathbb{C} \Gamma_{c}^{\prime \prime}$.

We produce $\Gamma_{c}^{(k)}$ from $\Gamma_{c}^{(k-1)}$ in the similar way. The sequence $\Gamma_{c}^{(k)}$ clearly stabilizes. Let $c^{(k)} \in \mathbb{C} \Gamma_{c}^{(k)}$ be a resulting parameter. Let $\lambda^{(k)} \in \mathfrak{h}^{*}$ be the parameter corresponding to $c^{(k)}$.

Lemma 5.6. We have $\lambda^{(k)} \in W^{a} \lambda$.

Proof. The root system for $\underline{W}_{1}^{a}$ is obtained from that for $W^{a}$ as the invariants under the action of a diagram automorphism group described in the proof of Lemma 5.4. It follows that $\underline{W}_{1}^{a}$ is a subgroup of $W^{a}$ and it acts on $\underline{\mathfrak{h}}_{1}^{*}$ as the subgroup. Therefore $\lambda^{\prime \prime} \in W^{a} \lambda$. Continuing to argue in the same way we see that $\lambda^{(k)} \in W^{a} \lambda$.

5.3. Proof of Theorem 5.1. The following proposition completes the proof of Theorem 5.1. Let us write $\Gamma_{c}$ for the stable normal subgroup $\Gamma_{c}^{(k)}$.

Proposition 5.7. Suppose that $\Gamma$ is solvable, equivalently, not of type $E_{8}$. The subgroup $\Gamma_{c} \subset \Gamma$ is a unique normal subgroup satisfying either of the following two properties:

(1) The image of $\overline{\mathrm{HC}}\left(\mathcal{A}_{\lambda}\right)$ in $\mathbb{C} \Gamma-\bmod$ is $\mathbb{C}\left(\Gamma / \Gamma_{c}\right)$-mod.

(2) $\Gamma_{c}$ is the minimal normal subgroup $\Gamma_{0}$ of $\Gamma$ such that there is a parameter $c^{0} \in \mathbb{C} \Gamma_{0}$ with the property that the corresponding parameter $\lambda^{0}$ lies in $W^{a} \lambda$.

Proof. Let us prove that the image of $\overline{\mathrm{HC}}\left(\mathcal{A}_{\lambda}\right)$ in $\mathbb{C} \Gamma$-mod under $\bullet+$ coincides with $\mathbb{C}\left(\Gamma / \Gamma_{c}\right)$-mod (which determines $\Gamma_{c}$ uniquely assuming such a subgroup exists). The proof is induction on the number of elements in $\Gamma$.

First of all, consider the situation when $\Gamma=\Gamma_{c}^{\prime}$. We claim that in this case the image of $\overline{\mathrm{HC}}\left(\mathcal{A}_{\lambda}\right)$ consists of the trivial representations. To prove this recall that the image is a tensor subcategory. If it contains some irreducible representation $V$ of $\Gamma$, then it contains all irreducible representations of $\Gamma / \Gamma_{0}$, where $\Gamma_{0}$ is the kernel of $\Gamma \rightarrow \operatorname{GL}(V)$. Since $\Gamma$ is solvable, we see that $\Gamma / \Gamma_{0}$ has a nontrivial one-dimensional representation. Then $\Gamma \neq \Gamma_{c}^{\prime}$ thanks to Proposition 5.3. We get a contradiction.

Now we can assume, by induction that our claim is proved for $\Gamma_{c}^{\prime}$ instead of $\Gamma$. Pick $\lambda^{\prime}$ as in Lemma 5.4. Then we have an equivalence $\operatorname{HC}\left(\mathcal{A}_{\lambda}\right) \cong \operatorname{HC}\left(\mathcal{A}_{\lambda^{\prime}}\right)$ that intertwines the embeddings of these categories into $\mathbb{C} \Gamma-\bmod$ by Lemma 4.9. Now for $\lambda^{\prime}$ instead of $\lambda$ our claim follows from Proposition 4.8 because $\mathcal{A}_{\lambda^{\prime}}$ is realized as $\Gamma / \Gamma_{c}^{\prime}$-invariants in the quantization of $\mathbb{C}^{2} / \Gamma_{c}^{\prime}$ with parameter $c^{\prime}$. This finishes the first characterization of $\lambda$.

Let us prove the characterization of $\Gamma_{c}$ in (2). Let $\Gamma_{0}$ be a normal subgroup such that there is $\lambda^{0} \in W^{a} \lambda$ with $c^{0} \in \mathbb{C} \Gamma_{0}$. Then $\mathbb{C}\left(\Gamma / \Gamma_{0}\right)$-mod lies in the image of $\operatorname{HC}\left(\mathcal{A}_{\lambda}\right)$ under • + As we have seen, this image coincides with $\mathbb{C}\left(\Gamma / \Gamma_{c}\right)$-mod. So $\Gamma_{c} \subset \Gamma_{0}$. And, by Lemma 5.6, we know that $W^{a} \lambda$ intersects $\left(\mathbb{C} \Gamma_{c}\right)^{\Gamma}$. 


\section{Classification For QuAntizations of SyMPleCtiC Singularities}

6.1. Structure of $\operatorname{HC}\left(\mathcal{A}_{\lambda}\right)$. In this section we consider a conical symplectic singularity $Y$ without dimension two slices of type $E_{8}$. Let, as before, $\Gamma$ denote the algebraic fundamental group of $Y^{r e g}$. Let $\Gamma_{i}, i=1, \ldots, k$, be the Kleinian groups corresponding to codimension 2 symplectic leaves so that we have group homomorphisms $\varphi_{i}: \Gamma_{i} \rightarrow \Gamma$. Inside $\Gamma_{i}$ we have a normal subgroup to be denoted by $\Gamma_{i, \lambda}$. Namely, let $\lambda_{i}$ be the component of $\lambda$ in $\mathfrak{h}_{i}^{*}$. We can produce $c_{i} \in \mathbb{C} \Gamma_{i}$ out of $\lambda_{i}$ and form the normal subgroup $\Gamma_{i, c_{i}} \subset \Gamma_{i}$ as in Proposition 5.7. To simplify the notation, we write $\Gamma_{i, \lambda}$ for $\Gamma_{i, c_{i}}$. Finally, let $\Gamma_{\lambda}$ denote the minimal normal subgroup of $\Gamma$ containing $\varphi_{i}\left(\Gamma_{i, \lambda}\right)$ for all $i$.

The following theorem is a direct corollary of Proposition 4.7 combined with Theorem 5.1. It strengthens Theorem 1.3 .

Theorem 6.1. The functor $\bullet$ † identifies $\overline{\mathrm{HC}}\left(\mathcal{A}_{\lambda}\right)$ with $\mathbb{C}\left(\Gamma / \Gamma_{\lambda}\right)$-mod.

6.2. Towards description of $\operatorname{HC}\left(\mathcal{A}_{\lambda^{\prime}}, \mathcal{A}_{\lambda}\right)$. Let as before $Y$ be a conical symplectic singularity. Let $\lambda, \lambda^{\prime} \in \mathfrak{h}_{Y}^{*}$. As was mentioned in the introduction, it makes sense to speak about $\mathrm{HC} \mathcal{A}_{\lambda^{\prime}}-\mathcal{A}_{\lambda}$-bimodules. Let $\operatorname{HC}\left(\mathcal{A}_{\lambda^{\prime}}, \mathcal{A}_{\lambda}\right)$ denote the category of such bimodules and $\overline{\mathrm{HC}}\left(\mathcal{A}_{\lambda^{\prime}}, \mathcal{A}_{\lambda}\right)$ denote the quotient by the full subcategory of bimodules with proper associated varieties.

One could ask to describe the category $\overline{\mathrm{HC}}\left(\mathcal{A}_{\lambda^{\prime}}, \mathcal{A}_{\lambda}\right)$ similarly to the description of $\overline{\mathrm{HC}}\left(\mathcal{A}_{\lambda}\right)$. The easiest case is when $\mathfrak{h}_{0}^{*}=\{0\}$, let us consider it first. Recall the weight lattice $\Lambda_{Y} \subset \mathfrak{h}_{Y}^{*}$, the image of $\operatorname{Pic}\left(X^{r e g}\right)$. Recall the extended affine Weyl group $W_{Y}^{a e}:=$ $W_{Y} \ltimes \Lambda_{Y}$.

Conjecture 6.2. Suppose that $\mathfrak{h}_{0}^{*}=\{0\}$. Then $\overline{\mathrm{HC}}\left(\mathcal{A}_{\lambda^{\prime}}, \mathcal{A}_{\lambda}\right) \neq\{0\}$ if and only if $\lambda^{\prime} \in$ $W_{Y}^{a e} \lambda$. Moreover, if $\lambda^{\prime} \in W_{Y}^{a e} \lambda$ the categories $\overline{\operatorname{HC}}\left(\mathcal{A}_{\lambda^{\prime}}, \mathcal{A}_{\lambda}\right), \overline{\operatorname{HC}}\left(\mathcal{A}_{\lambda}, \mathcal{A}_{\lambda^{\prime}}\right)$ contain mutually inverse objects. In particular, we have an equivalence $\overline{\mathrm{HC}}\left(\mathcal{A}_{\lambda^{\prime}}, \mathcal{A}_{\lambda}\right) \cong \overline{\mathrm{HC}}\left(\mathcal{A}_{\lambda}\right)$ of right $\overline{\mathrm{HC}}\left(\mathcal{A}_{\lambda}\right)$-module categories.

Let us explain an approach to this conjecture, where we have not worked out some technical details. It is easy to see that if $\lambda^{\prime} \in W_{Y}^{a e} \lambda$, then the conclusions of the conjecture hold (thanks to the translation bimodules introduced in Section 4.6). In fact, here we do not need to require that $\mathfrak{h}_{0}^{*}=\{0\}$, this restriction is only needed for the opposite direction. So what remains to show is that $\overline{\mathrm{HC}}\left(\mathcal{A}_{\lambda^{\prime}}, \mathcal{A}_{\lambda}\right) \neq\{0\}$ implies that $\lambda^{\prime} \in W_{Y}^{a} \lambda$. Using Proposition 3.1, we reduce the proof to the case when $Y=\mathbb{C}^{2} / \Gamma$. In this case (and in the more general case of symplectic quotient singularities), it was shown in [L3, Section 3.5] that $\overline{\mathrm{HC}}\left(\mathcal{A}_{\lambda^{\prime}}, \mathcal{A}_{\lambda}\right) \neq\{0\}$ implies that $\mathrm{HC}\left(\mathcal{A}_{\lambda^{\prime}}^{\text {reg }}, \mathcal{A}_{\lambda}^{\text {reg }}\right) \cong \mathbb{C} \Gamma$-mod (an equivalence of $\mathrm{HC}\left(\mathcal{A}_{\lambda^{\prime}}^{r e g}\right)-\mathrm{HC}\left(\mathcal{A}_{\lambda}^{r e g}\right)$-bimodule categories $)$. Then we can classify the irreducibles in $\overline{\mathrm{HC}}\left(\mathcal{A}_{\lambda^{\prime}}, \mathcal{A}_{\lambda}\right)$ using techniques similar to Sections 5.1 and 5.3 and the following easy observation: if $V$ lies in the image of $\overline{\mathrm{HC}}\left(\mathcal{A}_{\lambda^{\prime}}, \mathcal{A}_{\lambda}\right) \hookrightarrow \mathbb{C} \Gamma$-mod, then $V^{*}$ lies in the image of $\overline{\mathrm{HC}}\left(\mathcal{A}_{\lambda}, \mathcal{A}_{\lambda^{\prime}}\right)$ and hence $V^{*} \otimes V$ and $V \otimes V^{*}$ lie in the images of $\overline{\mathrm{HC}}\left(\mathcal{A}_{\lambda}\right)$ and $\overline{\mathrm{HC}}\left(\mathcal{A}_{\lambda^{\prime}}\right)$.

The case when $\mathfrak{h}_{0}^{*} \neq\{0\}$ is more difficult. There are examples where $\overline{\operatorname{HC}}\left(\mathcal{A}_{\lambda^{\prime}}, \mathcal{A}_{\lambda}\right) \neq\{0\}$ while $\lambda^{\prime} \notin W_{Y}^{a} \lambda$ (e.g. some cases of $\operatorname{Spec}(\mathbb{C}[\mathbb{O}])$, where codim $\overline{\bar{O}} \overline{\mathbb{O}} \backslash \mathbb{O} \geqslant 4$, this was studied in [L6]). A general reason for the complications is the difference between the groups $\operatorname{Pic}\left(\tilde{Y}^{0}\right)^{\Gamma}$ and $\operatorname{Pic}\left(Y^{r e g}\right)$ - the latter is a finite index subgroup in the former and may be proper. Because of this, for $\chi \in \operatorname{Pic}\left(\tilde{Y}^{0}\right)^{\Gamma}$ the translation bimodule $\tilde{\mathcal{A}}_{\lambda, \chi}^{0}$ carries only a projective representation of $\Gamma$. One should be able to prove that $\overline{\operatorname{HC}}\left(\mathcal{A}_{\lambda^{\prime}}, \mathcal{A}_{\lambda}\right) \neq\{0\}$ implies that the $\mathfrak{h}_{0}^{*}$-component $\lambda^{\prime}-\lambda$ lies in the image of $\operatorname{Pic}\left(\tilde{Y}^{0}\right)^{\Gamma}$. If that is the case, 
$\overline{\mathrm{HC}}\left(\mathcal{A}_{\lambda^{\prime}}, \mathcal{A}_{\lambda}\right)$ should embed into the category of projective $\Gamma$-representations with the Schur multiplier determined by $\lambda^{\prime}-\lambda$ (and equal to zero if the $\mathfrak{h}_{0}^{*}$-component of $\lambda^{\prime}-\lambda$ is in the image of $\operatorname{Pic}\left(Y^{r e g}\right)$ ). After that it should not be difficult to prove a result similar to Theorem 6.1.

\section{LUSZTIG'S QUOTIENT REVISITED}

7.1. Special orbits and quantizations with integral central character. Here we consider an important special case of the conical symplectic singularities: $Y:=\operatorname{Spec}(\mathbb{C}[\mathbb{O}])$, where $\mathbb{O}$ is a nilpotent orbit in a semisimple Lie algebra $\mathfrak{g}$. We will concentrate on the case when $\mathbb{O}$ is special. Let us recall what this means.

Pick Cartan and Borel subalgebras $\mathfrak{h} \subset \mathfrak{b} \subset \mathfrak{g}$. Let $W$ denote the Weyl group of $\mathfrak{g}$. Recall that the center of $U(\mathfrak{g})$ is identified with $\mathbb{C}\left[\mathfrak{h}^{*}\right]^{W}$ via the Harish-Chandra isomorphism. A two-sided ideal $\mathcal{J} \subset U(\mathfrak{g})$ is said to have integral central character if its intersection with the center is the maximal ideal in $\mathbb{C}\left[\mathfrak{h}^{*}\right]^{W}$ of a point in the weight lattice $\Lambda$. A nilpotent orbit $\mathbb{O}$ is called special if there is a two-sided ideal $\mathcal{J} \subset U(\mathfrak{g})$ with integral central character such that the associated variety of $U(\mathfrak{g}) / \mathcal{J}$ is $\overline{\mathbb{O}}$.

Now let $\mathcal{A}_{\lambda}$ is a filtered quantization of $Y$. As such, it comes equipped with a natural homomorphism $U(\mathfrak{g}) \rightarrow \mathcal{A}_{\lambda}$, see, e.g., [L8, Section 5]. Let $\mathcal{J}_{\lambda}$ denote the kernel. Clearly if $\mathcal{J}_{\lambda}$ has integral central character, then $\mathbb{O}$ is special. Conversely, we have the following result.

Proposition 7.1. Let $\mathbb{O}$ be a special orbit. There is $\lambda \in \mathfrak{h}_{Y}^{*}$ such that $\mathcal{J}_{\lambda}$ has integral central character if and only if $\mathbb{O}$ is not one of the following four special orbits (in the Bala-Carter notation):

(*) $A_{4}+A_{1}$ in $E_{7}$, and $A_{4}+A_{1}, E_{6}\left(a_{1}\right)+A_{1}, A_{4}+2 A_{1}$ in $E_{8}$.

This is [L6, Theorem 1.1]. In fact, one can find $\lambda$ explicitly, see the next section.

7.2. Computation of $\lambda$. Now we explain how to compute $\lambda \in \mathfrak{h}_{Y}^{*}$. For this we first need to recall results from [L8] on the computation of $\mathfrak{h}_{Y}^{*}$ and $W_{Y}$ for $Y=\operatorname{Spec}(\mathbb{C}[\mathbb{O}])$ for an arbitrary nilpotent orbit $\mathbb{O} \subset \mathfrak{g}$.

It was proved in [L8, Theorem 4.4] that $\mathbb{O}$ is birationally induced from a birationally rigid orbit $\mathbb{O}^{\prime}$ in a Levi subalgebra $\mathfrak{l}$ and the pair $\left(\mathfrak{l}, \mathbb{O}^{\prime}\right)$ is defined uniquely up to $G$ conjugacy. By definition, this means the following. Let $\mathfrak{l}$ be a Levi subalgebra and $\mathfrak{p}=\mathfrak{l} \ltimes \mathfrak{n}$ be a parabolic subalgebra with this Levi subalgebra. Let $\mathbb{O}^{\prime}$ be a nilpotent orbit in $\mathfrak{l}$. The subgroup $P \subset G$ acts on $Y^{\prime} \times \mathfrak{n}$, where we write $Y^{\prime}$ for $\operatorname{Spec}\left(\mathbb{C}\left[\mathbb{O}^{\prime}\right]\right)$. We have the generalized Springer morphism $G \times{ }^{P}\left(Y^{\prime} \times \mathfrak{n}\right) \rightarrow \mathfrak{g}$. It is easy to see that its image is the closure of a single orbit, say $\mathbb{O}$, called induced from $\left(\mathfrak{l}, \mathbb{O}^{\prime}\right)$. If the morphism $G \times{ }^{P}\left(Y^{\prime} \times \mathfrak{n}\right) \rightarrow \overline{\mathbb{O}}$ is birational, we say that $\mathbb{O}$ is birationally induced from $\left(\mathfrak{l}, \mathbb{O}^{\prime}\right)$. We say that $\mathbb{O}^{\prime}$ is birationally rigid if it cannot be birationally induced from a proper Levi subalgebra.

The following result is a special case of [L8, Proposition 4.6].

Lemma 7.2. We have $\mathfrak{h}_{Y}^{*}=\mathfrak{z}(\mathfrak{l})^{*}$ and $W_{Y}=N_{G}\left(\mathfrak{l}, \mathbb{O}^{\prime}\right) / L$.

Now we state the main result of this section. Let $\Delta^{+, \mathfrak{n}}$ denote the set of positive roots $\alpha$ such that the root space $\mathfrak{g}_{\alpha}$ is in $\mathfrak{n}$. 
Proposition 7.3. Suppose that $\mathbb{O}$ is special and is not one of the four orbits mentioned in Proposition 7.1. Set $\rho^{\prime}:=\frac{1}{2} \sum_{\alpha \in \Delta^{+, n}} \alpha$. Then $\mathcal{J}_{\rho^{\prime}}$ has integral central character.

Proof. It is known that $\mathbb{O}$ is induced from a birationally rigid special orbit $\mathbb{O}^{\prime} \subset \mathfrak{l}$, see [L6, Proposition 2.3]. By our additional restriction on $\mathbb{O}$, the unique filtered quantization $\mathcal{A}^{\prime}$ of $Y^{\prime}:=\mathbb{C}\left[\mathbb{O}^{\prime}\right]$ has integral central character, [L6, Theorem 1.1].

Consider the homogeneous space $G / N$ and its sheaf of differential operators, $D_{G / N}$. So we get a sheaf of algebras $D_{G / N} \otimes \mathcal{A}^{\prime}$ on $G / N$. The group $L$ acts on $D_{G / N} \otimes \mathcal{A}^{\prime}$ in a Hamiltonian way, the quantum comoment map is the sum of the quantum comoment maps $\Phi_{D, \eta}, \Phi_{\mathcal{A}}$ for the actions of $L$ on $D_{G / N}$ and on $\mathcal{A}^{\prime}$. Here $\eta \in\left(\mathfrak{l}^{*}\right)^{L}$. These quantum comoment maps are as follows. We set $\Phi_{D, \eta}(x):=x_{G / N}-\langle\eta, x\rangle$, and $\Phi_{\mathcal{A}}$ is chosen so that it vanishes of $\mathfrak{z}(\mathfrak{l})$. We then can form the quantum Hamiltonian reduction of $D_{G / N} \otimes \mathcal{A}^{\prime}$ with respect to the $L$-action getting a sheaf of filtered algebras on $G / P$ to be denoted by $D^{\eta}\left(G, P, \mathcal{A}^{\prime}\right)$. This sheaf can be viewed as a filtered quantization of $G \times{ }^{P}\left(Y^{\prime} \times \mathfrak{n}\right)$.

We claim that the quantization $D^{-\rho^{\prime}}\left(G, P, \mathcal{A}^{\prime}\right)$ has period zero. Thanks to [L4, Theorem 5.4.1] this follows once we show that

(a) the quantization $D_{G / N} \otimes\left[\left.\mathcal{A}^{\prime}\right|_{\left(Y^{\prime}\right)^{r e g}}\right]$ of $T^{*}(G / N) \times\left(Y^{\prime}\right)^{r e g}$ is even (meaning that it is the specialization at $h=1$ of a graded formal quantization that is even in the sense of [L4, Section 2.3])

(b) and the quantum comoment map $\Phi_{D,-\rho^{\prime}}+\Phi_{\mathcal{A}}: \mathfrak{l} \rightarrow D_{G / N} \otimes \mathcal{A}^{\prime}$ is symmetrized in the terminology of [L4, Section 5.4].

To prove (a) we notice that both factors $D_{G / N}$ and $\left.\mathcal{A}^{\prime}\right|_{\left(Y^{\prime}\right)^{r e g}}$ have period zero (for the former this follows from [L4, Section 5]). A similar argument proves (b).

So $H^{0}\left(G / P, \mathcal{D}^{\eta}\left(G, P, \mathcal{A}^{\prime}\right)\right) \cong \mathcal{A}_{\eta+\rho^{\prime}}$. It remains to prove that the kernel $\mathcal{J}_{\rho^{\prime}}$ of $U(\mathfrak{g}) \rightarrow$ $H^{0}\left(G / P, \mathcal{D}^{0}\left(G, P, \mathcal{A}^{\prime}\right)\right)$ has integral central character. Let $\mathfrak{m}^{\prime}$ denote the intersection of the kernel of $U(\mathfrak{l}) \rightarrow \mathcal{A}^{\prime}$ with the center of $U(\mathfrak{l})$. Then we have an algebra homomorphism $\mathcal{D}^{0}\left(G, P, U(\mathfrak{l}) / \mathfrak{m}^{\prime}\right) \rightarrow \mathcal{D}^{0}\left(G, P, \mathcal{A}^{\prime}\right)$. The algebra $U(\mathfrak{l}) / \mathfrak{m}^{\prime}$ is the algebra of twisted differential operators on $P / B$ with twist, say $\mu$, that must be integral because the kernel of $U(\mathfrak{l}) \rightarrow$ $\mathcal{A}^{\prime}$ has integral central character. It is easy to see that $H^{0}\left(G / P, \mathcal{D}^{0}\left(G, P, U(\mathfrak{l}) / \mathfrak{m}^{\prime}\right)\right) \cong$ $D^{\mu}(G / B)$. So the homomorphism $\mathcal{U} \rightarrow H^{0}\left(G / P, \mathcal{D}^{0}\left(G, P, \mathcal{A}^{\prime}\right)\right)$ factors through $\mathcal{U} \rightarrow$ $D^{\mu}(G / B)$ and hence the kernel has integral central character.

7.3. Lusztig's quotient vs $\Gamma_{\lambda}$. The following proposition describes the Lusztig quotient.

Proposition 7.4. Let $\mathbb{O}$ be as in Proposition 7.3. Let $\lambda \in \mathfrak{h}_{Y}^{*}$ be such that $\mathcal{J}_{\lambda}$ has integral central character. Then the Lusztig quotient $\mathbf{A}_{c}$ coincides with $\Gamma / \Gamma_{\lambda}$.

Proof. The proof is in several steps. Let us start by relating the construction of the present paper to that of [LO].

Step 1. We note that the ideal $\mathcal{J}_{\lambda}$ is completely prime and has central character, so is primitive. Consider the $\mathrm{W}$-algebra $\mathcal{W}$ constructed from the orbit $\mathbb{O}$. In [L1] (see, for example, Theorem 1.2.2 there) the author produced an $\mathbf{A}(\mathbb{O})$-orbit of finite dimensional irreducible representations of $\mathcal{W}$ starting from a primitive ideal in $U(\mathfrak{g})$. In our case, $U(\mathfrak{g}) / \mathcal{J}_{\lambda}$ has multiplicity 1 on $\mathbb{O}$. It follows that the orbit consists of a single 1 -dimensional representation. 
Step 2. Consider the category $\mathrm{HC}\left(U(\mathfrak{g}) / \mathcal{J}_{\lambda}\right)$ of the $\mathrm{HC} U(\mathfrak{g})$-bimodules annihilated by $\mathcal{J}_{\lambda}$ on the left and on the right and its full subcategory $\operatorname{HC}^{\partial \mathbb{O}}\left(U(\mathfrak{g}) / \mathcal{J}_{\lambda}\right)$ of bimodules supported on $\partial \mathbb{O}$. Let $\mathrm{HC}_{\mathbb{O}}\left(U(\mathfrak{g}) / \mathcal{J}_{\lambda}\right)$ denote the quotient category. The inclusion $U(\mathfrak{g}) / \mathcal{J}_{\lambda} \hookrightarrow \mathcal{A}_{\lambda}$ gives rise to the forgetful functor $\operatorname{HC}\left(\mathcal{A}_{\lambda}\right) \rightarrow \operatorname{HC}\left(U(\mathfrak{g}) / \mathcal{J}_{\lambda}\right)$, which, in turn, induces a functor $\overline{\mathrm{HC}}\left(\mathcal{A}_{\lambda}\right) \rightarrow \mathrm{HC}_{\mathbb{O}}\left(U(\mathfrak{g}) / \mathcal{J}_{\lambda}\right)$. We claim that this functor is an equivalence. For this we consider the functor $\bullet$ from [L1, Section 3.4]. By [L1, Theorem 1.3.1], this functor identifies $\mathrm{HC}_{\mathbb{O}}\left(U(\mathfrak{g}) / \mathcal{J}_{\lambda}\right)$ with a full subcategory of the category $\operatorname{Bim}^{Q}\left(\mathcal{W} / \mathcal{I}_{\lambda}\right)$ of $Q$-equivariant $\mathcal{W} / \mathcal{I}_{\lambda}$-bimodules. Here the notation is as follows. By $\mathcal{I}_{\lambda}$ we denote the image of $\mathcal{J}_{\lambda}$ under $\bullet$, this is a two-sided ideal of codimension 1 . Finally, $Q$ is the reductive part of the centralizer $Z_{G}(e)$ of $e \in \mathbb{O}$, where $G$ is the adjoint group with Lie algebra $\mathfrak{g}$. The group $Q$ acts on $\mathcal{W}$ in a Hamiltonian way. The functor $\bullet_{\dagger}: \operatorname{HC}\left(U(\mathfrak{g}) / J_{\lambda}\right) \rightarrow \operatorname{Bim}^{Q}\left(\mathcal{W} / \mathcal{I}_{\lambda}\right)$ has a right adjoint functor $\bullet^{\dagger}$ that is also a left inverse for the functor $\mathrm{HC}_{\mathbb{O}}\left(U(\mathfrak{g}) / J_{\lambda}\right) \rightarrow \operatorname{Bim}^{Q}\left(\mathcal{W} / \mathcal{I}_{\lambda}\right)$. It was checked in [L8, Lemma 5.2] that $\mathcal{A}_{\lambda}=\left(\mathcal{W} / \mathcal{I}_{\lambda}\right)^{\dagger}$. This implies that $\overline{\mathrm{HC}}\left(\mathcal{A}_{\lambda}\right) \stackrel{\sim}{\rightarrow} \mathrm{HC}_{\mathbb{O}}\left(U(\mathfrak{g}) / \mathcal{J}_{\lambda}\right)$.

Step 3. Note that $Q / Q^{\circ}=\Gamma / \pi_{1}(G)$. Below we will write $\underline{\Gamma}$ for $Q / Q^{\circ}$. Clearly, $\operatorname{Bim}^{Q}\left(\mathcal{W} / \mathcal{I}_{\lambda}\right)$ is identified with $\mathbb{C} \underline{\Gamma}$-mod. Comparing the constructions of $\bullet \dagger$ in [L1, Section 3.4] and in the present paper we see that the embedding $\overline{\mathrm{HC}}\left(\mathcal{A}_{\lambda}\right) \hookrightarrow \mathbb{C} \Gamma$-mod described above in this step coincides with what we have constructed in Section 4.3. In particular, $\Gamma_{\lambda}$ contains $\pi_{1}(G)$. Let us write $\underline{\Gamma}_{\lambda}$ for $\Gamma_{\lambda} / \pi_{1}(G)$.

Step 4. Our goal is to show that $\underline{\Gamma}_{\lambda}$ coincides with the kernel of $\underline{\Gamma} \rightarrow \overline{\mathbf{A}}_{c}$. First of all, note that $\left|\overline{\mathbf{A}}_{c}\right|=\left|\underline{\Gamma} / \underline{\Gamma}_{\lambda}\right|$. Indeed, both numbers are equal to the number of simples in $\overline{\mathrm{HC}}\left(\mathcal{A}_{\lambda}\right)$ : for the left hand side this follows from [LO, Theorem 1.1], while for the right hand side this is a consequence of Theorem 6.1. Now the case when $\overline{\mathbf{A}}_{c}$ is not abelian is easy: in all such cases this group coincides with $\Gamma$.

Step 5. Now let us assume that $\overline{\mathbf{A}}_{c}$ is abelian. This is always the case when $\mathfrak{g}$ is classical. If $\mathfrak{g}$ is exceptional and $\overline{\mathbf{A}}_{c}$ is abelian, then $\overline{\mathbf{A}}_{c} \cong \mathbb{Z} / 2 \mathbb{Z}$, see, e.g. [LO, Section 6.7]. It was shown in [LO, Sections 6.5-6.7] that there is a finite dimensional irreducible representation of the central reduction $\mathcal{W}_{\rho}$ whose stabilizer in $\underline{\Gamma}$ is the kernel of $\underline{\Gamma} \rightarrow \overline{\mathbf{A}}_{c}$, to be denoted by $\underline{\Gamma}^{0}$. Let $\mathcal{J}_{\rho}^{\prime}$ be the corresponding primitive ideal in $U(\mathfrak{g})$. It follows from [LO, Sections $7.4,7.5]$ that the quotient of the category of $\mathrm{HC} U(\mathfrak{g}) / \mathcal{J}_{\rho}^{\prime}-U(\mathfrak{g}) / \mathcal{J}_{\lambda}$-bimodules modulo the bimodules supported on $\partial \mathbb{O}$ is equivalent to Vect. The image of this quotient category in the category of semisimple finite dimensional $Q$-equivariant $\mathcal{W}$-bimodules is closed under tensoring by the images under $\bullet \nmid$ of objects in $\operatorname{HC}\left(\mathcal{U}_{\lambda} / \mathcal{J}_{\lambda}\right)$. These images are precisely the representations of $\underline{\Gamma} / \underline{\Gamma}_{\lambda}$ by Theorem 1.3 . As a right module category over $\mathbb{C} \underline{\Gamma}$-mod, the quotient category has the form $\operatorname{Rep}^{\psi} \underline{\Gamma}^{0}$, where $\psi$ is some Schur multiplier. It follows from [LO, Remark 7.7] and the existence of an A(O)-stable one-dimensional representation of $\mathcal{W}$ with integral central character (that follows from [L6, Theorem 1.1]) that $\psi$ is a coboundary. So we have an irreducible representation $V \in \operatorname{Rep}\left(\underline{\Gamma}^{0}\right)$ with the following property: $V \otimes U \cong \underline{\Gamma}^{0} V^{\oplus \operatorname{dim} U}$ for any representation $U$ of $\underline{\Gamma} / \underline{\Gamma}_{\lambda}$.

Step 6. If $\overline{\mathbf{A}}_{c}$ is abelian, then so is $\underline{\Gamma}$. This can be seen from explicit computations of $\overline{\mathbf{A}}_{c}$, see, e.g., [LO, Section 6.7], and the tables from [C, Section 13.3]. All irreducible representations of $\underline{\Gamma}^{0}$ are 1-dimensional. So the condition $V \otimes U \cong{ }_{\Gamma^{0}} V^{\oplus \operatorname{dim} U}$ implies that $U$ must be trivial over $\underline{\Gamma}^{0}$ and hence $\underline{\Gamma}^{0} \subset \underline{\Gamma}_{\lambda}$. But the cardinalities of these two groups are the same by Step 4 and so we get $\underline{\Gamma}_{0}=\underline{\Gamma}_{\lambda}$. 
Remark 7.5. In $[\mathrm{Lu}]$ Lusztig observed that $\overline{\mathbf{A}}_{c}$ coincides with $\mathbf{A}\left(\mathbb{O}_{1}\right)$ for a suitable nilpotent orbit $\mathbb{O}_{1}$ in a Levi subalgebra $\mathfrak{l}_{1}$. His proof is a case by case argument. We would like to sketch how this result follows from Proposition 7.4 .

First of all, note that if $\mathbb{O}$ is birationally induced from $\left(\mathfrak{l}_{1}, \mathbb{O}_{1}\right)$, then $\mathbb{O} \hookrightarrow G \times{ }^{P_{1}}\left(\mathbb{O}_{1} \times \mathfrak{n}_{1}\right)$, which gives an epimorphism $\pi_{1}(\mathbb{O}) \rightarrow \pi_{1}\left(G \times{ }^{P_{1}}\left(\mathbb{O}_{1} \times \mathfrak{n}_{1}\right)\right) \cong \pi_{1}\left(\mathbb{O}_{1}\right)$.

Identify $\mathfrak{z}(\mathfrak{l})$ with $\mathfrak{z}(\mathfrak{l})^{*}$ via the Killing form of $\mathfrak{g}$. Now pick a small complex neighborhood $U$ of $\lambda \in \mathfrak{h}_{Y}^{*}=\mathfrak{z}(\mathfrak{l})^{*}$. The locus of $\lambda^{\prime} \in U$ with $\Gamma_{\lambda^{\prime}}=\Gamma_{\lambda}$ has the form $(\lambda+\Pi) \cap U$ for a uniquely determined vector subspace $\Pi \subset \mathfrak{z}(\mathfrak{l})^{*}$. Take the centralizer of $\Pi \subset \mathfrak{z}(\mathfrak{l})$ in $\mathfrak{g}$ for $\mathfrak{l}_{1}$. For $\mathbb{O}_{1}$ we take the orbit in $\mathfrak{l}_{1}$ induced from $\left(\mathfrak{l}^{\prime}, \mathbb{O}^{\prime}\right)$. Then one can see that $\mathbf{A}\left(\mathbb{O}_{1}\right)=\underline{\Gamma} / \underline{\Gamma}_{\lambda}$.

Remark 7.6. Let us explain how to handle the remaining four orbits. Three of them: $A_{4}+A_{1}$ in $E_{7}, E_{8}$ and $A_{4}+2 A_{1}$ have codimension of the boundary $\geqslant 4$. In this case, [L6, Proposition 4.7] implies that $\overline{\mathbf{A}}=\mathbf{A}(\mathbb{O})$ (all these orbits are birationally rigid so [L6, Proposition 4.7] formally applies but, if fact, the proof only uses the condition on the codimension of the boundary). In all these cases, $\mathbf{A}(\mathbb{O})$ is $\mathbb{Z} / 2 \mathbb{Z}$.

Let us explain how to handle the remaining case, $E_{6}\left(a_{1}\right)+A_{1}$ in $E_{8}$. Here $\mathbf{A}(\mathbb{O}) \cong$ $\mathbb{Z} / 2 \mathbb{Z}$ as well. This orbit is birationally induced from $A_{4}+A_{1}$ in $E_{7}$. Thanks to [LO, Theorem 1.1], the claim that $\overline{\mathbf{A}}=\mathbf{A}(\mathbb{O})$ is equivalent to the existence of a 1-dimensional representation of $\mathcal{W}$ with integral central character that is not $\mathbf{A}(\mathbb{O})$-stable. Such a representation, say $\underline{N}$, exists for the orbit $\mathbb{O}$ of type $A_{4}+A_{1}$ in $E_{7}$ because $A_{4}+A_{1}$ is Richardson. Take such a representation and extend it to a representation of the $\mathrm{W}$-algebra for the corresponding Levi subalgebra, which amounts in specifying the character, say $\chi$, of the action of the center of the Levi. Then we can induce the resulting 1-dimensional representation, see [L2, Section 6], to get a representation, say $N_{\chi}$, of the $\mathrm{W}$-algebra $\mathcal{W}$ for $\mathbb{O}$. When $\chi$ is integral, $N_{\chi}$ has integral central character. On the other hand, the set of all $\chi$ such that $N_{\chi}$ is $\mathbf{A}(\mathbb{O})$-stable is Zariski closed. It is not difficult, but somewhat technical, to show that if $N_{\chi}$ is $\mathbf{A}(\mathbb{O})$-stable for all $\chi$, then $\underline{N}$ is $\mathbf{A}(\mathbb{O})$-stable. We arrive at a contradiction that shows that $N_{\chi}$ is not $\mathbf{A}(\mathbb{O})$-stable for some integral $\chi$.

\section{REFERENCES}

[B] A. Beauville, Symplectic singularities. Invent. Math. 139 (2000), no. 3, 541-549.

[BG] J. Bernstein, S. Gelfand. Tensor products of finite and infinite dimensional representations of semisimple Lie algebras. Compositio Mathematica, 41(1980), n.2, p. 245-285.

[BK] R. Bezrukavnikov, D. Kaledin, Fedosov quantization in the algebraic context. Moscow Math. J. 4 (2004), 559-592.

[BPW] T. Braden, N. Proudfoot, B. Webster, Quantizations of conical symplectic resolutions I: local and global structure. Astérisque 384 (2016), 1-73.

[Br] A. Broer, Decomposition varieties in semisimple Lie algebras. Canad. J. Math. 50 (1998), no. 5, 929-971.

[C] R. Carter, Finite groups of Lie type. Conjugacy classes and their characters. J. Wiley and sons, 1985.

[CM] D. Collingwood, W. McGovern, Nilpotent orbits in semisimple Lie algebras. Chapman and Hall, London, 1993.

[CBH] W. Crawley-Boevey, M. Holland. Noncommutative deformations of Kleinian singularities. Duke Math. J. 92(1998), 605-635.

[EG] P. Etingof and V. Ginzburg. Symplectic reflection algebras, Calogero-Moser space, and deformed Harish-Chandra homomorphism, Invent. Math. 147 (2002), 243-348.

[GL] I. Gordon, I. Losev, On category $\mathcal{O}$ for cyclotomic rational Cherednik algebras. J. Eur. Math. Soc. 16 (2014), 1017-1079. 
[Gr] A. Grothendieck. Cohomologie locale de faisceaux cohérent et théorèmes de Lefschetz locaux et globaux, North Holland, 1968.

[H] M. Holland. Quantization of the Marsden-Weinstein reduction for extended Dynkin quivers. Ann. Sci. Ec. Norm. Super. IV Ser. 32(1999), 813-834.

$[\mathrm{K}]$ D. Kaledin. Symplectic singularities from the Poisson point of view. J. Reine Angew. Math. 600(2006), 135-156.

[L1] I. Losev, Finite dimensional representations of W-algebras. Duke Math J. 159(2011), n.1, 99-143.

[L2] I. Losev, 1-dimensional representations and parabolic induction for $W$-algebras. Adv. Math. 226(2011), 6, 4841-4883.

[L3] I. Losev, Completions of symplectic reflection algebras. Selecta Math., 18(2012), N1, 179-251.

[L4] I. Losev, Isomorphisms of quantizations via quantization of resolutions. Adv. Math. 231(2012), 12161270.

[L5] I. Losev. Bernstein inequality and holonomic modules (with a joint appendix by I. Losev and P. Etingof). Adv. Math. 308 (2017), 941-963.

[L6] I. Losev. Quantizations of regular functions on nilpotent orbits. Bull. Inst. Math. Acad. Sin. 13 (2018), n.2, 199-225.

[L7] I. Losev. Wall-crossing functors for quantized symplectic resolutions: perversity and partial Ringel dualities. PAMQ, 13 (2017), n.2, 247-289.

[L8] I. Losev, Deformations of symplectic singularities and Orbit method for semisimple Lie algebras. arXiv: 1605.00592 .

[L9] I. Losev, Derived equivalences for Symplectic reflection algebras. Int. Math. Res. Notices, rnz178.

[LMBM] I. Losev, L. Mason-Brown, D. Matvieievskyi, On unipotent Harish-Chandra bimodules. In preparation.

[LY] I. Losev, S. Yu, On Harish-Chandra modules over quantizations of nilpotent orbits. In preparation.

[LO] I. Losev, V. Ostrik, Classification of finite dimensional irreducible modules over $W$-algebras. Compos. Math. 150(2014), N6, 1024-1076.

[Lu] G. Lusztig. Characters of reductive groups over a finite field, Ann. Math. Studies 107, Princeton University Press (1984).

[N1] Y. Namikawa, Extension of 2-forms and symplectic varieties. J. Reine Angew. Math. 539 (2001), 123-147.

[N2] Y. Namikawa, Poisson deformations of affine symplectic varieties, II. Kyoto J. Math. 50 (2010), no. 4, 727752 .

[N3] Y. Namikawa, Poisson deformations of affine symplectic varieties. Duke Math. J. 156 (2011), no. 1, 51-85.

[N4] Y. Namikawa, Fundamental groups of symplectic singularities. Higher dimensional algebraic geometryin honour of Professor Yujiro Kawamata's sixtieth birthday, 321334, Adv. Stud. Pure Math., 74, Math. Soc. Japan, Tokyo, 2017.

[P] A. Premet. Special transverse slices and their enveloping algebras. Adv. Math. 170(2002), 1-55.

[PT] A. Premet, L. Topley. Derived subalgebras of centralizers and finite $W$-algebras. Compos. Math. 150 (2014), 1485-1548.

[S] J. Simental, Harish-Chandra bimodules over rational Cherednik algebras. Adv. Math. 317 (2017), 299-349.

[V] D. Vogan, Dixmier algebras, sheets, and representation theory. Operator algebras, unitary representations, enveloping algebras, and invariant theory (Paris, 1989), 333-395, Progr. Math., 92, Birkhuser Boston, Boston, MA, 1990.

[W] B. Webster, A categorical action on quantized quiver varieties. Math. Z. 292 (2019), no. 1-2, 611-639.

Department of Mathematics, Yale University, CT, USA

E-mail address: ivan.loseu@gmail.com 